\title{
ULTRASOUND DETECTION USING OPTICAL INTERFEROMETRY
}

\author{
by \\ Nusrat Jahan Surovy \\ Master of Science, University of Dhaka, Bangladesh \\ 2011

\begin{abstract}
A thesis
in partial fulfillment of the

requirements for the degree of

Master of Science

in the Program of

Biomedical Physics
\end{abstract} \\ presented to Ryerson University
}

Toronto, Ontario, Canada 2017

@ Nusrat Surovy 2017 


\section{AUTHOR'S DECLARATION}

I hereby declare that I am the sole author of this thesis. This is a true copy of the thesis, including any required final revisions, as accepted by my examiners.

I authorize Ryerson University to lend this thesis to other institutions or individuals for the purpose of scholarly research.

I further authorize Ryerson University to reproduce this thesis by photocopying or by other means, in total or in part, at the request of other institutions or individuals for the purpose of scholarly research.

I understand that my thesis may be made electronically available to the public. 
Ultrasound detection using optical interferometry

Master of Science 2017

Nusrat Jahan Surovy

Biomedical Physics

Ryerson University

\begin{abstract}
Ultrasound imaging is a widely used noninvasive imaging technique for biomedical and other applications. Piezoelectric devices are commonly used for the generation and detection of ultrasound in these applications. However, implementation of twodimensional arrays of piezoelectric transducers for 3D ultrasound imaging is complex and expensive. Optical Fabry-Perot interferometry is an attractive alternative to the piezoelectric devices for detection of ultrasound. In this method a thin film etalon is constructed and used. Light reflected from the two surfaces of this thin film produces an intensity which depends on the film thickness. When ultrasound is incident on the film, it changes the thickness of the film and consequently modulates the light intensity on the film. In our work, we made two types of etalon (Finesse 2) for our experiment. We detected lower frequency ultrasound ( $0.5 \mathrm{MHz}$ or $1 \mathrm{MHz})$ using the build etalon. We determined a linear relationship between the strength of the optical signals and the exerted pressure on a film by the ultrasound. The dependence of the etalon performance on the light wavelength was demonstrated indirectly by measuring the signal at various light incidence angle. Simulation results are also presented. Lastly, we proposed the optimum design of this detection system based on the simulation results. This method of ultrasound detection can be a potential low-cost approach for 3D ultrasound imaging.
\end{abstract}




\section{ACKNOWLEDGEMENTS}

I would like to express my deepest gratitude to my Supervisor Dr. Yuan $\mathrm{Xu}$ and CoSupervisor Dr. Michael Kolios for believing in my quality, pursuing me as a Master's student, and handling me with great patience for more than 3.5 years. Their continuous guidance, inspiration, and support helped me to finish this degree. I would also like to thank Dr. Alexandre Douplik, Dr. Carl Kumaradas, Dr. Vladislav Toronov and Dr. Xijia Gu for being the members of my supervisory committee in various seasons.

I would like to thank my co-workers Dr. Krishnan Sathiyamoorthy, Aditya Pandya, Dr. Borna Maraghechi, and friends Ryan West, Nauman Mufti (UofT) for their help during my lab work. I would also like to express thanks to my special friends Jagdish Bhatt, Ping Gong and Reyhaneh Nosrati for their supportive friendship. I am also grateful to all the staff of the Physics Department including Arthur Worthington and Sophia Finos.

I would like to thank my husband, my back-supporter, Dr. Muhammad Zulfiker Alam, the most significant supporter during my M.Sc. study period. Without his support this degree would not have been possible to achieve. He helped me and also blessed me with a baby during this M.Sc. degree.

I am grateful to my brother Dr. Shahadate Ahmed Rezvy for his supportive contribution in my final semester. I am also grateful to my all siblings including Dr. Shahjada Ahmed Pahlovy, Dr. Sarifun Naher and Kamrun Nahar Begum for their unforgettable support in my past. Finally, I would like to thank my parents with the deepest respect, whom I did not meet during my M.Sc. period but their continuous inspiration encouraged me to carry on this study and finish this degree finally. 


\section{Dedication}

To my Husband

DR. MUHAMMAD ZULFIKER ALAM 


\section{Contents}

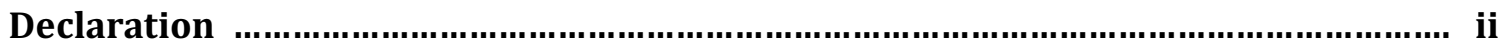

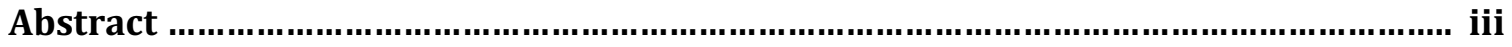

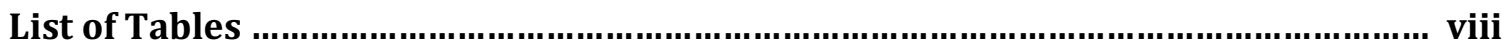

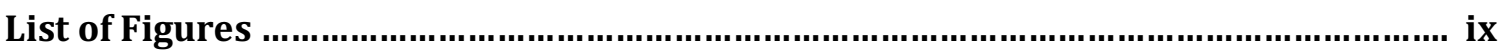

1 Chapter 1 INTRODUCTION........................................................................... 1

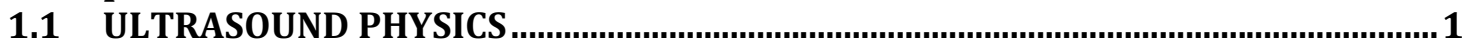

1.2 HISTORY OF ULTRASOUND

1.3 LIMITATIONS OF ULTRASOUND IMAGING

1.4 OPTICAL DETECTION OF ULTRASOUND

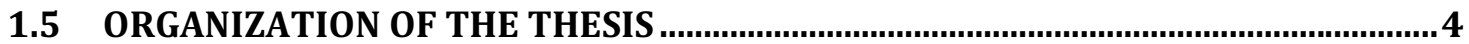

2 Chapter 2 ULTRASOUND DETECTION USING OPTICAL ETALON ...................... 6

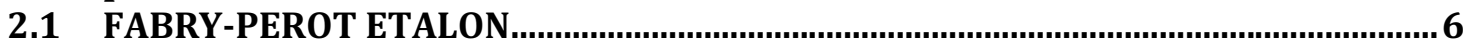

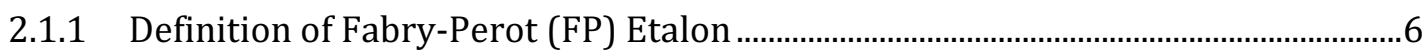

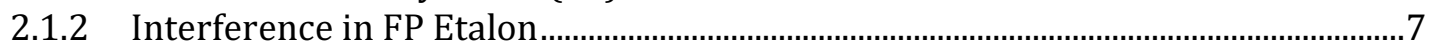

2.2 APPLICATION OF FP ETALON IN ULTRASOUND DETECTION .............................. 14

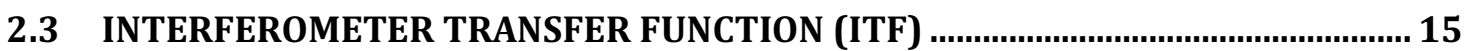

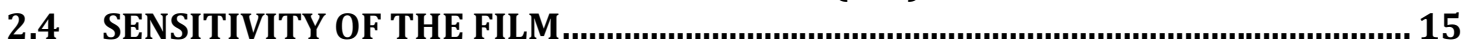

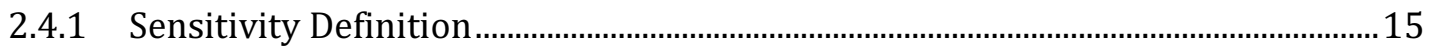

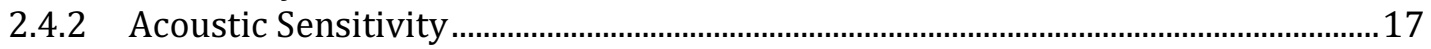

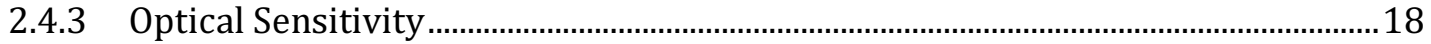

2.4.4 Practical Sensitivity Parameters................................................................................. 19

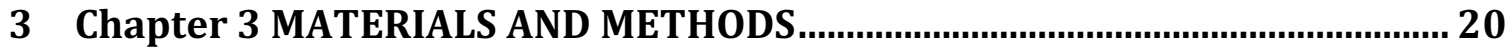

3.1 FABRICATION OF ETALON 20

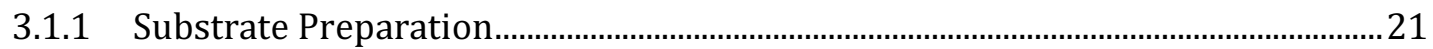

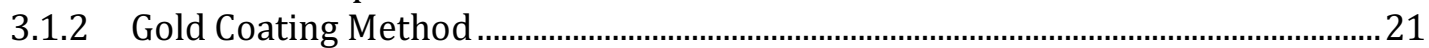

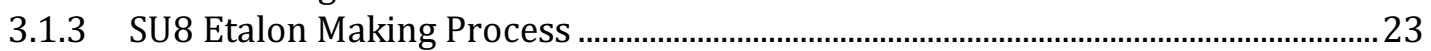

3.1.4 Parylene-C Etalon Making Process ................................................................................25

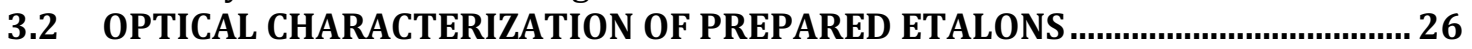

3.3 EXPERIMENTAL SET-UP FOR OPTICAL DETECTION OF ULTRASOUND ............. 27

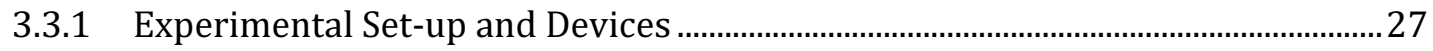

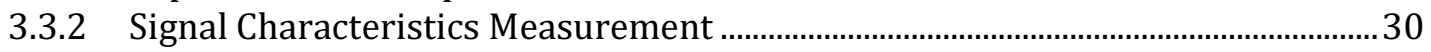

3.4 OPTICAL DETECTION OF DIFFERENT FREQUENCY ULTRASOUND....................... 31

3.5 OPTICAL SIGNAL STRENGTH VARIATION WITH FILM PRESSURE ...................... 31

3.6 EXPERIMENTAL SET-UP FOR OBLIQUE LIGHT INCIDENCE SYSTEM …................. 32

3.6.1 Experiment in a Smaller Light Incident Angle Range using Five Layer Film .......34

3.6.2 Experiment in a Smaller Light Incident Angle Range using Four Layer Film .....34

3.6.3 Experiment in a Larger Light Incident Angle Range using Five Layer Film .........34

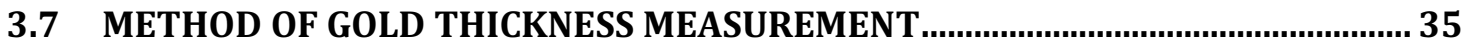

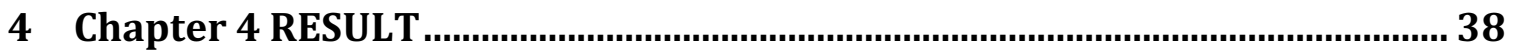

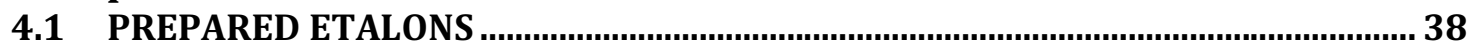

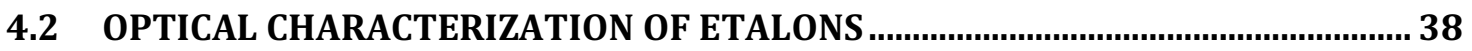

4.3 OPTICAL DETECTION OF ULTRASOUND ............................................................ 42

4.4 OPTICAL DETECTION OF DIFFERENT FREQUENCY ULTRASOUND.......................44 
4.5 OPTICAL SIGNAL STRENGTH VARIATION WITH INCIDENCE PRESSURE ON

ETALON

4.6 REFLECTED LIGHT INTENSITY VS OBLIQUE LIGHT INCIDENCE : THEORETICAL

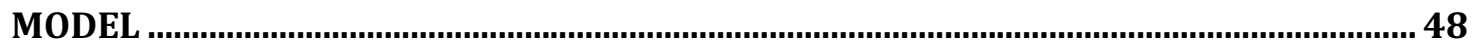

4.7 REFLECTED LIGHT INTENSITY VS OBLIQUE LIGHT INCIDENCE:

EXPERIMENTAL RESULT ............................................................................................... 52

4.7.1 Experiment in a Smaller Light Incident Angle Range using Five Layer Film ..........52

4.7.2 Experiment in a Smaller Light Incident Angle Range using Four Layer Film ..........53

4.7.3 Experiment in a Larger Light Incident Angle Range using Five Layer Film ............ 54

4.8 REFLECTED LIGHT INTENSITY VS WAVELENGTH: THEORETICAL

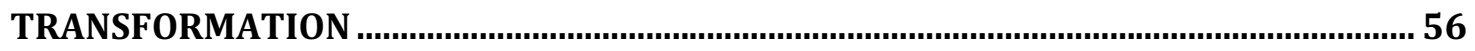

REFLECTED LIGHT INTENSITY VS WAVELENGTH (NORMAL LIGHT INCIDENCE):

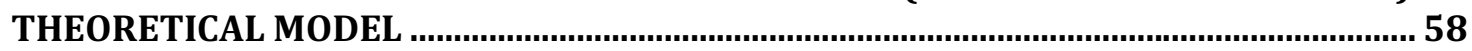

4.9.

4.10 REFLECTED LIGHT INTENSITY VS WAVELENGTH (NORMAL LIGHT

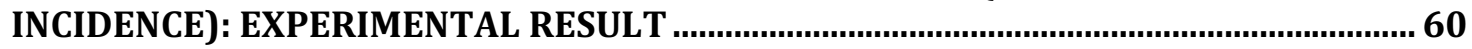

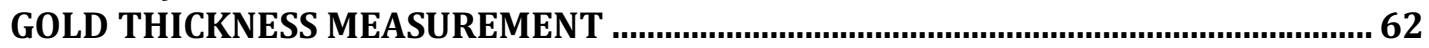

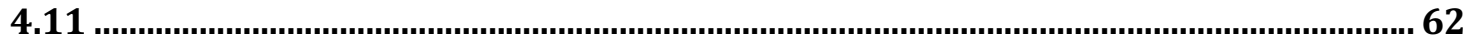

4.11.1 Resistance Measurement of All Films by Four-Probe Technique ..........................62

4.11.2 Reference Film Thickness Measurement by AFM Technique................................. 63

4.11.3 Correlation between 'Gold Coating Time' and 'Gold Thickness' .............................64

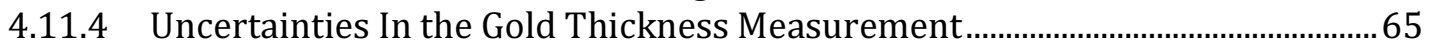

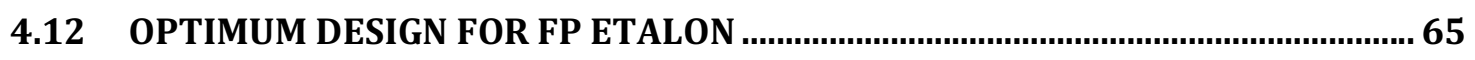

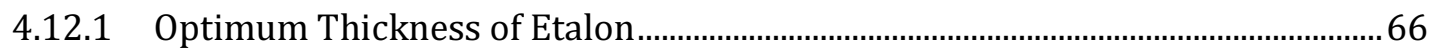

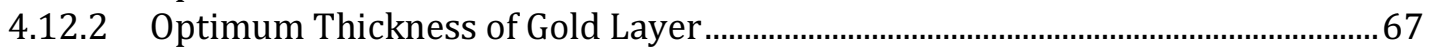

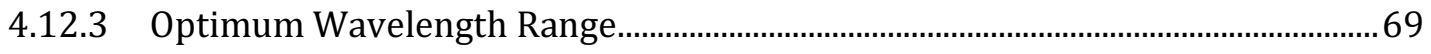

5 Chapter 5 DISCUSSION, CONCLUSION AND FUTURE WORK ……...................... 72

5.1 DISCUSSION

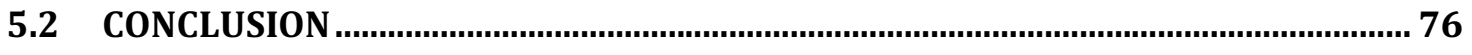

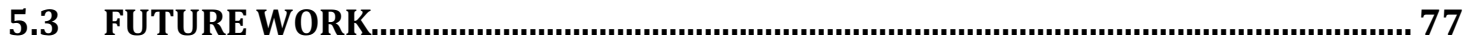

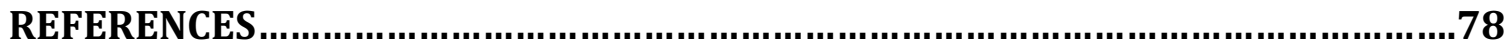




\section{List of Tables}

Table 3.1 Description of the devices and material models.....................................28

Table 3.2 Description of the transducers.............................................................. 31

Table 4.1 Summary result of ultrasound detection by etalons.................................44

Table 4.2 Result of optical detection of ultrasound using different frequencies .......46

Table 4.3 Various samples, their time of gold coating, and their measured resistance

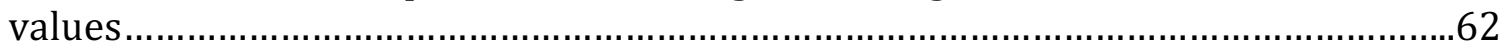

Table 4.4 Gold thickness measurement result of reference film (160s gold coating)

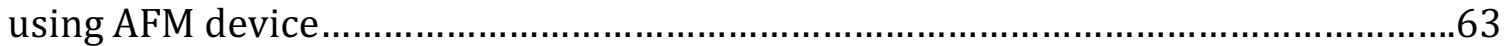

Table 4.5 Table for polymer thickness, gold thickness, and maximum derivative.....68

Table 4.6 Final optimization results for a Fabry-Perot device...................................70 


\section{List of Figures}

Figure 2.1 A typical Fabry-Perot etalon with thickness $l$ and refractive index $n$ showing the multiply reflected $\left(\mathrm{R}_{0}, \mathrm{R}_{1}, \mathrm{R}_{2}, ..\right)$ and transmitted $\left(\mathrm{T}_{1}, \mathrm{~T}_{2}, ..\right)$ rays produced in the two sides of a Fabry-Perot etalon. Both transmitted and refracted rays are produced from a single incident ray with $\theta$ degree transmission angle [24] .............6 Figure 2.2 Multiple beam interference for a parallel surface film............................. Figure 2.3 Transmission vs phase graph which follows Airy function........................11 Figure 2.4 Reflection vs phase graph which follows (1-Airy function) .......................11

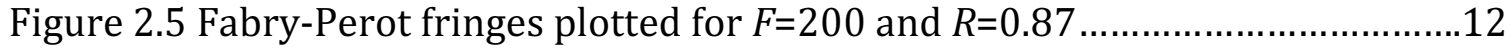
Figure 2.6 (a) Ultrasound is incident on a polymer etalon from its right side. (b) The thickness of the polymer etalon is modified due to the incident ultrasound pressure wave; and the ultrasound wave is reflected (c) A probing laser is incident from the left side of the etalon and later it is reflected from the two surfaces of the deformed etalon

Figure 2.7 Interferometer transfer function (Here, $\left.\Phi=\phi_{0}+d \phi\right)[10] \ldots \ldots \ldots \ldots \ldots \ldots . . . . .15$ Figure 2.8 Diagram showing a polymer sensing film, where a laser light (shown by red line) incident and later reflected from the two surfaces of the film. The blue curves showing the successive internal reflection of acoustic wave inside the film..16 Figure 3.1 Schematic diagram for the etalon.......................................................20 Figure 3.2 (a) Schematic diagram for a sputtering process (b) Sputter device [44]..22 Figure 3.3 Spin coater device [43] ...................................................................23 Figure 3.4 (a) Schematic diagram of vacuum evaporation technique [41]

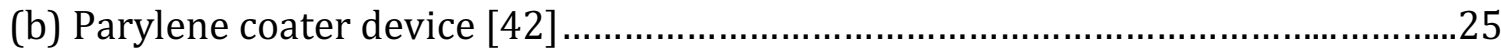

Figure 3.5 Schematic diagram of the full experimental set-up.................................. 27 Figure 3.6 (a) Schematic diagram (b) A picture of the major portion of our

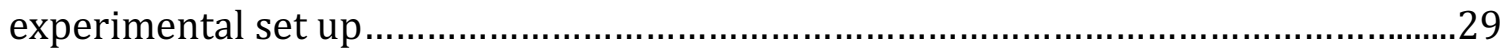

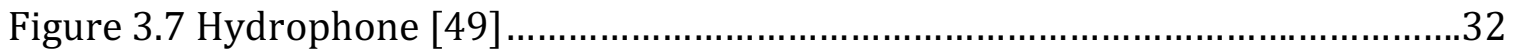

Figure 3.8 Angular incidence system of laser beam using goniometer...................... 33 Figure 3.9 (a) Five layer and (b) Four layer Parylene etalons.............................. 34

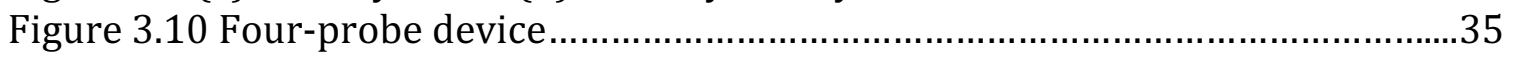

Figure 3.11 AFM device .................................................................................... 35

Figure 3.12 (a) A picture taken by the inspection camera showing gold sample where a rectangular hole was created by a rectangular laser pulse on the gold coated-uncoated boundary line. Region 1 and 2 are two selected AFM scan area on two sides of this laser generated hole. (b) AFM scanned picture to find the glass depth (c) AFM scanned picture to find glass with gold layer depth............................36 Figure 4.1 Sample of prepared (a) SU8 and (b) Parylene-C etalon.............................38 Figure 4.2 Transmission spectrum of the SU8 etalon which was coated with gold for 138 seconds in the sputter device. 39 Figure 4.3 Transmission spectrum of the Parylene-C etalon, which was coated with gold for 200 seconds in the sputter device. 39

Figure 4.4 1MHz ultrasound signal in water.......................................................................42 Figure 4.5 Optical signal recorded using the SU8 etalon (gold coated for $138 \mathrm{~s}$ )........43 
Figure 4.6 Optical signal recorded using the Parylene-C etalon (gold coated for 200

s)

Figure 4.7 Pulse echo ultrasound signals (a, b, c) of frequency $1 \mathrm{MHz}, 0.5 \mathrm{MHz}$ and

$0.5 \mathrm{MHz}$ and their corresponding optical detection signals $(\mathrm{d}, \mathrm{e}, \mathrm{f}$ )... . .45

Figure 4.8 (a) Optical signal amplitude vs pulser voltage (b) Pressure vs pulser voltage (c) Optical signal amplitude vs pressure graph.

Figure 4.9 Fresnel coefficients vs light incident angle for p polarized light. The coefficient of reflection, transmission and absorption are presented by red, blue and green curves.

Figure 4.10 Fresnel coefficients vs light incident angle for s polarized light. The coefficient of reflection, transmission and absorption are presented by red, blue and green curves.

Figure 4.11 Reflected light amplitude vs. light incident angle result using five layer Parylene film in the light incident angle range (33-53) degree. The solid and dotted lines are representing two times measurements of same experiment on same condition .53

Figure 4.12 Reflected light amplitude vs light incident angle result using four layer Parylene film in the light incidence angle range (33-53) degree. The solid and dotted lines represent two times measurements of same experiment under same condition where the Parylene films position was slightly varied in second time .54

Figure 4.13 Reflected light amplitude vs light incident angle using a five layer Parylene film interferometer in the light incident angle range of 8-82 degrees...........55 Figure 4.14 Reflected light intensity vs wavelength plot obtained from Fig 4.13 through a theoretical transformation.

Figure 4.15 Simulation model of 'Reflection vs. wavelength' for nearly normal incidence light (p-polarized case)

Figure 4.16 Reflectivity measurement of two types of Parylene etalons by spectrometer. The solid and dotted lines are representing the measurement results using five layer and four layer etalon respectively. 61 Figure 4.17 (a) A line profile on the scanning region 1 showing the height of glass substrate's upper surface to the bottom of rectangular laser pulse created hole $\left(\mathrm{h}_{1}=0 \mathrm{~nm}-(-400 \mathrm{~nm})=400 \mathrm{~nm}\right)$. (b) Line profile of the scanning region 2 showing the height of gold layer's upper surface to the bottom of rectangular laser pulse created hole $\left(\mathrm{h}_{2}=0 \mathrm{~nm}-(-500 \mathrm{~nm})=500 \mathrm{~nm}\right)$.

Figure 4.18 A graph showing a linear relationship between 'Gold coating time' and the measured 'Gold layer thickness'..

Figure 4.19 A diagram showing the measurement uncertainty due to the inclined incidence of laser. 65

Figure 4.20 Change of etalon thickness vs etalon thickness graph for Parylene considering the reflectivity of the etalon as 0.9 (ultrasound frequency $1 \mathrm{MHz}$ ).........66 Figure 4.21 Sample plots for $29 \mathrm{~nm}$ gold thickness: (a) Reflection coefficient vs wavelength graph for Parylene film interferometer. (b) Zoom in view of (a); (c) First derivative of reflection coefficient vs wavelength graph; (d) Zoom in view of (c).......67 Figure 4.22 Maximum derivative of reflection coefficients vs Gold thickness graph, plotted for $541.9 \mu \mathrm{m}$ Parylene film. 
Figure 4.23 Optimum wavelength vs Gold thickness graph showing the optimum wavelength range $(600 \mathrm{~nm}-1000 \mathrm{~nm})$ plotted for $541900 \mathrm{~nm}$ thick, and 0.9 reflectivity Parylene film interferometer.

Figure 5.1 (a) SNR vs. averaging number plot (b) SNR vs. square root of averaging number plot.

Figure 5.2 Normalized plot of reflected light intensity vs. light incident angle............73 Figure 5.3 (a) A view from the top of our experimental arrangement (b) Circular transducer surface and lines representing the laser beam paths. The line 'Position 1' corresponds to a laser beam path going through the edge of circular transducer surface. 'Position 3' corresponds to a laser beam path along a line through the diameter of the circular surface. 'Position 2' corresponding a laser beam path between positions 1 and 3 .

Figure 5.4 (a) Graphical result of optical signal amplitude vs transducer surface and light beam distance (b) A general sound intensity profile of a focused transducer 


\section{Chapter 1 INTRODUCTION}

In modern biomedical science the major existing techniques for biomedical imaging are X-ray, magnetic resonance imaging (MRI), computed tomography (CT) and ultrasound imaging. Each of these techniques provides unique contrast and has both advantages and disadvantages. Among all these techniques, ultrasound imaging is the most popular because it is non-invasive and radiation free, inexpensive, portable and real time. This makes ultrasound imaging easy to use for doctors and accessible to patients.

\subsection{ULTRASOUND PHYSICS}

Ultrasound imaging is an imaging technique where high frequency sound waves are used to visualize internal organs of the body. Ultrasounds are mechanical waves, which have frequencies above the human hearing range, i.e. frequencies more than $20 \mathrm{kHz}$. In practice medical professionals use ultrasound frequencies between $2 \mathrm{MHz}$ to $15 \mathrm{MHz}$ [3], far higher frequencies than the human hearing range. Ultrasound can propagate through solid and liquid mediums like soft tissues, fluids and bones. When ultrasound travels through these mediums, it interacts with the medium particles and the medium particles oscillate around their mean position. During the interaction with the medium particles, ultrasound becomes reflected, scattered (mostly) and absorbed.

If the incident ultrasound has a sound pressure $P$, and particle velocity $v$, then they are related as:

$$
P=\rho v_{s} v=Z v
$$

Equation 1.1

Where, $\rho$ is the density of the medium, $v_{s}$ is the speed of sound and $\mathrm{Z}=\rho v_{s}$, where $Z$ is the acoustic impedance of the medium [6]

When ultrasound transmits through the material, the reflection and scattering by the material particles produces an echo signal. The echoes are produced only when the 
transmitting wave experienced a mismatch of acoustic properties at the boundary. The higher the mismatch of acoustic impedance produces stronger echoes. These echoes are used to make the ultrasound images. The other information that is used in imaging is returning time $(t)$ of the echoes. Using a simple formula, $S=v_{s} t / 2$, the distance of the internal organ $(S)$ is estimated. Thus an image of the internal body part is created.

Ultrasound is produced from a transducer. A transducer is mainly formed by Piezoelectric crystals. When a voltage is applied on the piezoelectric crystal, the crystal can generate pressure waves; also, the crystals respond to an external acoustic field by generating a voltage. These criteria are the generation and detection mechanism of ultrasound by a piezoelectric transducer.

\subsection{HISTORY OF ULTRASOUND}

After World War II, piezoelectric crystals like quartz were started to be used [4]. At that time it was used in sonar, and in some devices to detect defects in metals. Their successful application made physicists interested to apply ultrasound in medical sectors. K.T. Dussik [5] in 1941 first suggested in his paper that ultrasound can be used for diagnostic purposes. Since 1954 ultrasound was used in Japan, US and Sweden. Thus using ultrasound in medicine became more widespread. Instead of quartz, more suitable materials like lead zirconate are used now. In less than 80 years of ultrasound history, transducer and imaging system become better developed, popular but still suffering low contrast noise ratio in soft tissues.

\subsection{LIMITATIONS OF ULTRASOUND IMAGING}

The spatial resolution of ultrasound imaging improves with larger probe aperture. Ultrasound imaging aperture can be increased in two ways [2]. The first one is mechanically scan a single element transducer and the second way is using $2 \mathrm{D}$ or $3 \mathrm{D}$ transducer arrays. The advantage of scanning a single element transducer is the capability of producing high frequency wave like $50 \mathrm{MHz}$ and above. The spacing between the elements can be made by the spacing distance of scanning which can be reduced to few 
microns. The low f-number [50] of these high frequency transducers reduces the depth of the field. A mechanical scanning tool needs to attach with this transducer to operate the system. The process is lengthy as scanning takes so much time. Due to these disadvantages, it is inconvenient to use scanned single elements clinically.

In a $1 \mathrm{D}$ or $2 \mathrm{D}$ array system, one of the main problems is their fabrication, as dicing piezoceramics to the micron level is very difficult. Furthermore, to operate these systems a wire connection is required to each element connected to the associated electronics, which makes the system extremely difficult to assemble and bulky. Real-time 3D imaging with large number of elements is very difficult to realize with piezoelectric technology.

Contrast in optical imaging systems is based on the optical properties of the biological tissues. Optical properties in biological tissues can provide rich contrast at specific wavelengths. Due to the difference of optical properties in biological tissues, there is also significant scattering of light, which results in poor resolution deep in tissues [1]. Therefore optical imaging at large depth is difficult to achieve. The ultrasound waves have much weaker scattering in tissues so its resolution in sufficient depth is satisfactorily, but its contrast noise ratio is poor.

\subsection{OPTICAL DETECTION OF ULTRASOUND}

One of the simplest and effective optical methods of detecting ultrasound waves is using a Fabry-Perot interferometer [6, 8-14]. A Fabry-Perot interferometer or etalon consists of two plane parallel, highly reflective mirror in two sides of a transparent material. Light from an external source incident in one surface of the etalon undergoes multiple reflections within the etalon. The parallel transmitted and reflected rays in two sides of the etalon produce interference. A resonance condition occurs when optical path length is integral multiples of the wavelength. Reflected intensity becomes almost zero near the resonance condition. This resonance can be used to detect ultrasound. The ultrasound pressure that is exerted on an etalon changes its thickness and therefore the resonance condition also changes. The resonance condition changes modulate the intensity changes 
of the reflected beam. Measuring the reflected output intensity provides the information of the ultrasound pressure that was exerted on the etalon. Thus, ultrasound is detected using optical techniques. At the wavelength where the derivative of the intensity over the wavelength is largest, there is the largest sensitivity for the etalon to detect ultrasound.

The biggest advantage of this technique is its sensing area, the focal spot of the laser beam which can be easily reduced to a few microns using conventional optics. This focal spot can be thought of as an element in a array transducer. The spacing of an array can be controlled using the same convenient way too. In this system, there is no need of complex electric circuits. This technique also free from electrical interference which is considered as a problem in piezoelectric techniques, especially at higher frequencies. Also by beam splitting, an array can be formed and complicated dicing can be avoided. On the other side, when a large or unfocused beam is used, we can map the pressure in a line or area for 2D and 3D imaging [15-16].

The Fabry-Perot technique is not applied in medical sector yet as it is still in research period and only few groups are currently working on it. Most of the groups are working with high frequency detection [17-21, 54-57]. Ultrasound frequency is highly related to ultrasound imaging depth. The lower the frequency, the higher the imaging depth. So imaging research in depth of human body, using lower frequency ultrasound is still a potential sector of research. In this thesis, we tried to do some ultrasound detection work using lower (1 MHz and less than $1 \mathrm{MHz}$ ) frequency ultrasound.

\subsection{ORGANIZATION OF THE THESIS}

In this first chapter I presented the limitations of existing ultrasound imaging techniques and introduced the hybrid technique of ultrasound detection. In the second chapter, I described the theoretical background related to the proposed technique. In chapter three I presented the materials and experimental methods. I made two types of multilayer etalon with two different materials for my experiment. The etalons were made of SU8 and Parylene-C materials and they were prepared in two different processes such as spin coating and vacuum evaporation respectively. To improve the etalon surfaces reflectivity 
I used gold coating through sputter deposition system. I made two different experimental arrangements where light could incident on a film in normal and oblique way. For the both arrangements a horizontally set transducer and an etalon were used in water. Ultrasound produced from the transducer hits the etalon and changed its thickness in micron level. A laser was set outside the water tank and opposite side of the etalon which was directed towards the etalon to incident onto it. The etalon had gold coating so it reflected the light clearly and the reflected light was captured by a diode. Necessary optical informations were received through the diode. Different transducers were used to produce different frequency ultrasound. I measured how much pressure an etalon experiences when ultrasound is incident on it. To know this I used a hydrophone (hydrophone membrane) in place of the etalon and measured the pressure experienced by it. The method where I used oblique light incidence, I used there a goniometer to measure the light incidence and reflection angle. The system I used for gold coating was initially unable to provide informations about its exact thickness, so I made different thicknesses of gold coating using different exposure time. Later I measured gold thickness using 4probe and AFM devices. Some simulations and optimizations were done using professor A. Dahlin's code [38]. In chapter four I presented my experimental results and the analysis. I verified the optical signals from the etalon which were induced by the incident ultrasound at various ultrasound frequencies. A linear relationship was found between the strength of the optical signals and the exerted pressure on the film by the ultrasound. An etalon performance depends on the light wavelength. Usually a tunable continuous laser is needed to set the etalon at the best performance point. However, this type of laser was not available in the lab. So the dependence of the etalon performance on the light wavelength was demonstrated indirectly by measuring the signal at various light incidence angles. This was possible because the interferometer output is a function of light wavelength and light incidence angle in the form of phase difference $\delta$ only. Simulation results were also presented. Lastly, in chapter four I proposed the optimum design of this detection system based on the simulation results. In chapter five I discussed the results, limitations of the technique and future directions for this research. 


\section{Chapter 2 ULTRASOUND DETECTION USING OPTICAL ETALON}

\subsection{FABRY-PEROT ETALON}

\subsubsection{Definition of Fabry-Perot (FP) Etalon}

A Fabry-Perot interferometer [22-24] consists of two plane parallel highly reflecting surfaces separated by a small adjustable distance $(l)$ of about several millimeters to several centimeters. A parallel surface transparent film with partially transparent metal coating (gold/silver or aluminum) in its two sides is called Fabry-Perot etalon, which has a fixed thickness. The purpose of metal coating is to improve the reflectivity of two parallel surfaces that increases the multiple reflections within the etalon (Figure 2.1)

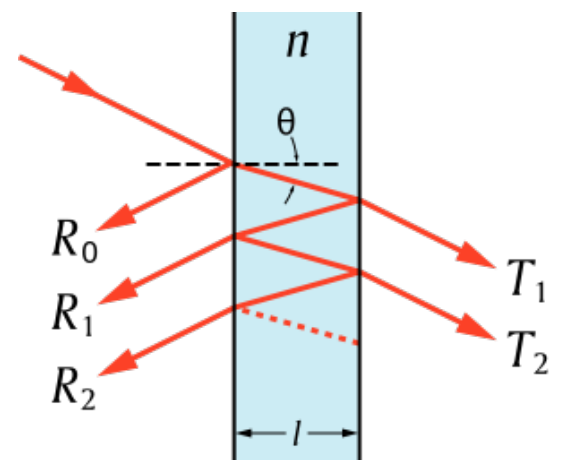

Figure 2.1 A typical Fabry-Perot etalon with thickness $l$ and refractive index $n$ showing the multiply reflected $\left(\mathrm{R}_{0}, \mathrm{R}_{1}, \mathrm{R}_{2}, ..\right)$ and transmitted $\left(\mathrm{T}_{1}, \mathrm{~T}_{2}, ..\right)$ rays produced in the two sides of a Fabry-Perot etalon. Both transmitted and refracted rays are produced from a single incident ray with $\theta$ degree transmission angle [24]

The Fabry-Perot device was first developed by Charles Fabry and Alfred Perot in 1899. The difference between an ordinary parallel surface thin film and a Fabry-Perot is in the reflectivity of the two surfaces. Fabry-Perot device has two surfaces with much higher reflectivity which causes multiple reflection within the etalon, and in consequence the reflection and transmission light intensities in two sides of the device become much more sensitive to the wavelength and the film thickness than an ordinary thin film. 
Fabry-Perot etalons are mainly used in telecommunication, lasers and spectroscopy [24]. Fused silica or diamond etalon are used in telecommunication networks. It is also used in optical wavemeters and optical spectrum analyzers to determine wavelength of light with great precision. Laser resonator uses etalon to change the laser from multi-mode to single- mode. Recently its use is started in medical research to improve some medical technologies [6, 8-14].

\subsubsection{Interference in FP Etalon}

Consider a non-absorbing transparent thin film of thickness $l$ [22] with two parallel surfaces kept in a transparent media with film refracting index $\mathrm{n}$. (Figure 2.2). The film is kept in a medium with refracting indexes $n_{1}$ and $n_{2}$ in its two side $\left(\mathrm{n}_{1}=\mathrm{n}_{2}\right)$.

Lets represent the amplitude-transmission and reflection coefficients by the letters ' $t$ ' and ' $r$ ' respectively.

$\mathrm{t}=$ The fraction of the amplitude of a wave transmitted upon on entering the film

$\mathrm{t}^{\prime}=$ The fraction transmitted when the wave exits the film

$r=$ The fraction of the amplitude of a wave reflected upon entering into the film

r'=The fraction reflected when the waves exits the film

We consider the film is illuminated by a broad source. For our convenience, we here will consider only one ray of wavelength $\lambda$ from this broad source, which will trace through the film.

Let $E_{0}$ is the amplitude of initial incoming wave (Figure 2.2). Let the incident wave be represented by $E_{0} e^{i \omega t_{m}}$. 


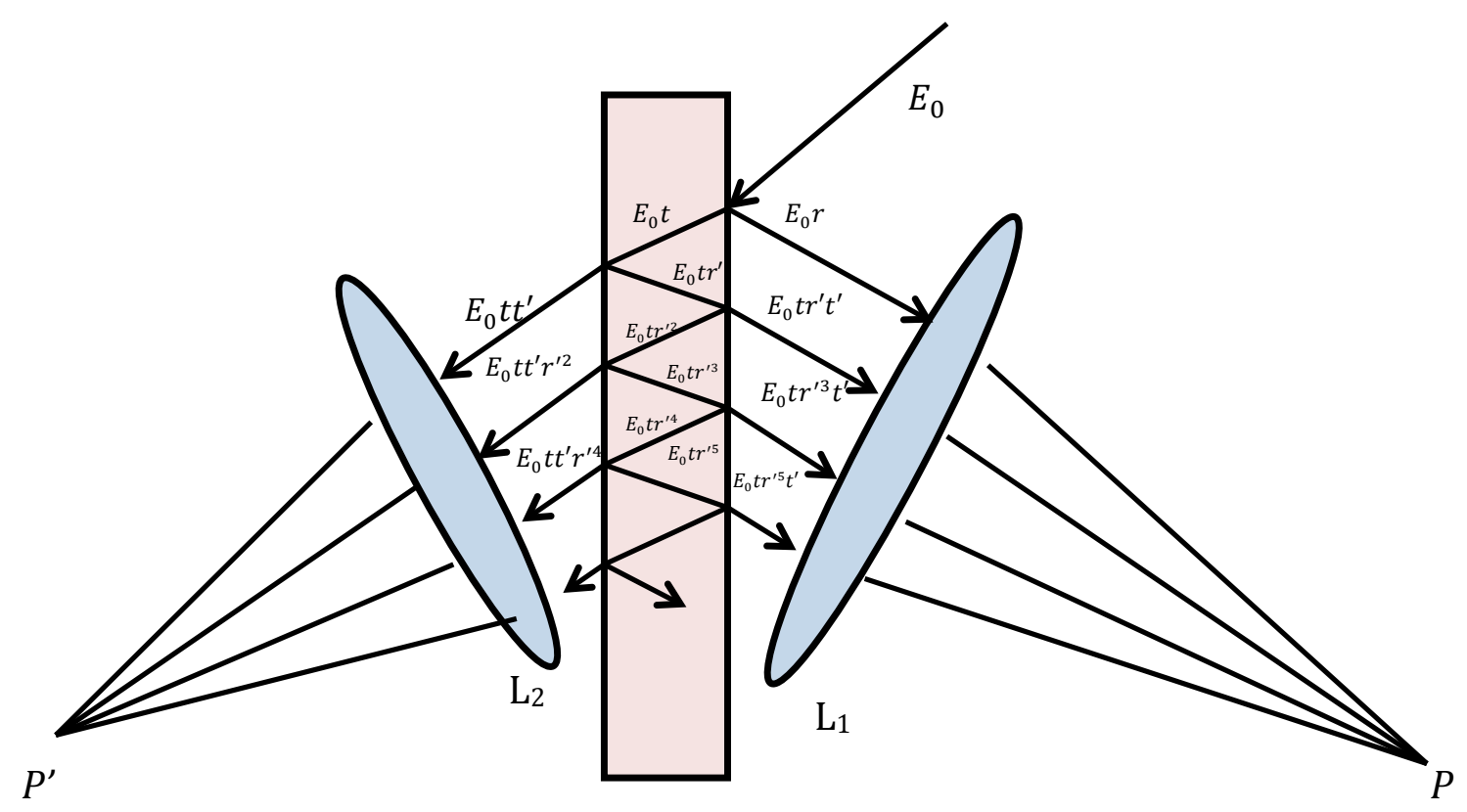

Figure 2.2 Multiple beam interference for a parallel surface film

The $E_{0} e^{i \omega t_{m}}$ incident wave produces multiple, parallel reflected waves in the same side of incident wave. The parallel reflected rays are collected with a lens $L_{1}$, which focuses the rays at point $P$. The optical fields at point $P$ are given by:

$$
\begin{gathered}
E_{1 r}=E_{0} r e^{i \omega t_{m}} \\
E_{2 r}=E_{0} t r^{\prime} t^{\prime} e^{i\left(\omega t_{m}-\delta\right)} \\
E_{3 r}=E_{0} t r^{\prime 3} t^{\prime} e^{i\left(\omega t_{m}-2 \delta\right)} \\
\ldots \ldots \ldots \ldots \ldots \ldots \\
\cdots \ldots \ldots \ldots \ldots \cdots \\
E=E_{0} t r^{\prime(2 N-3)} t^{\prime} e^{i\left[\omega t_{m}-(N-1) \delta\right]}
\end{gathered}
$$

Here $\delta, 2 \delta \ldots \ldots . .(\mathrm{N}-1) \delta$ are the contrubutions to the phase from the optical path length difference between two adjacent rays. If $\theta$ is an angle the light travels through the film then the phase difference $\delta$ is associated with the light wavelength $\lambda$ and transmission angle $\theta$ (in film media) by the following equation $[24,51]$ :

$$
\delta=\left(\frac{2 \pi}{\lambda}\right) 2 n l \cos \theta
$$


The resultant reflected scalar wave at point $P$ is:

$$
E_{r}=E_{1 r}+E_{2 r}+E_{3 r} \ldots \ldots \ldots+E_{N r}
$$

Upon substitution the values of each term of Equation 2.2, we get,

$$
\begin{gathered}
E_{r}=E_{0} r e^{i \omega t_{m}}+E_{0} t r^{\prime} t^{\prime} e^{i\left(\omega t_{m}-\delta\right)}+E_{0} t r^{\prime} t^{\prime} e^{i\left(\omega t_{m}-\delta\right)} \\
+\ldots .+E_{0} t r^{\prime(2 N-3)} t^{\prime} e^{i\left[\omega t_{m}-(N-1) \delta\right]}
\end{gathered}
$$

This is also written as

$$
E_{r}=E_{0} e^{i \omega t_{m}}\left\{r+r^{\prime} t t^{\prime} e^{-i \delta}\left[1+\left(r^{\prime 2} e^{-i \delta}\right)+\left(r^{\prime 2} e^{-i \delta}\right)^{2}+\cdots \ldots \ldots \ldots+\left(r^{\prime 2} e^{-i \delta}\right)^{N-2}\right]\right\}
$$

Equation 2.3

If, $\left[r^{\prime 2} e^{-i \delta}\right]<1$ and if the number of terms in the series approaches infinity, the previous equation becomes,

$$
E_{r}=E_{0} e^{i \omega t_{m}}\left[r+\frac{r^{\prime} t t^{\prime} e^{-i \delta}}{1-r^{\prime 2} e^{-i \delta}}\right]
$$

Equation 2.4

If we neglect absorption, we can use the relation, $r=-r^{\prime}$ and $t t^{\prime}=1-r^{2}$. Then the above equation becomes,

$$
E_{r}=E_{0} e^{i \omega t_{m}}\left[\frac{r\left(1-e^{-i \delta}\right)}{1-r^{2} e^{-i \delta}}\right]
$$

The reflected flux density at $P$ is given by:

$$
I_{r}=\frac{E_{r} E_{r}^{*}}{2}
$$

Using Equation 2.5 in above equation, we obtained:

$$
I_{r}=\frac{E_{0}^{2} r^{2}\left(1-e^{-i \delta}\right)\left(1-e^{+i \delta}\right)}{2\left(1-r^{2} e^{-i \delta}\right)\left(1-r^{2} e^{+i \delta}\right)}
$$

Which is transformed to:

$$
I_{r}=I_{i} \frac{2 r^{2}(1-\cos \delta)}{\left(1+r^{4}\right)-2 r^{2} \cos \delta}
$$

$\left[I_{i}=E_{0}^{2} / 2=\right.$ incident flux density $]$

The parallel transmitted waves are shown in the left side of Figure 2.2 and collected by a lens $\mathrm{L}_{2}$ to focus at point P'. Similar way, we can write for the amplitude of these transmitted waves: 


$$
\begin{gathered}
E_{1 t}=E_{0} t t^{\prime} e^{i \omega t_{m}} \\
E_{2 t}=E_{0} t t^{\prime} r^{\prime 2} e^{i\left(\omega t_{m}-\delta\right)} \\
E_{3 t}=E_{0} t t^{\prime} r^{\prime 4} e^{i\left(\omega t_{m}-2 \delta\right)} \\
\ldots \ldots \ldots \ldots \ldots \ldots \\
\cdots \ldots \ldots \ldots \ldots \ldots \\
E_{N t}=E_{0} t t^{\prime} r^{\prime 2(N-1)} e^{i\left[\omega t_{m}-(N-1) \delta\right]}
\end{gathered}
$$

The total transmitted wave becomes

$$
E_{t}=E_{0} e^{i \omega t_{m}}\left[\frac{t t^{\prime}}{1-r^{2} e^{-i \delta}}\right]
$$

Equation 2.9

Multiplying Equation 2.9 by its complex conjugate, and using the relation $I_{t}=E_{t} E_{t}^{*} / 2$, $I_{i}=E_{0}^{2} / 2 ;$ we obtained

$$
I_{t}=\frac{I_{i}\left(t t^{\prime}\right)^{2}}{\left(1+r^{4}\right)-2 r^{2} \cos \delta}
$$

Using the trigonometric relation, $\cos \delta=1-2 \sin ^{2}\left(\frac{\delta}{2}\right)$, the above equation becomes,

$$
I_{t}=I_{i} \frac{1}{1+\left[2 r /\left(1-r^{2}\right)\right]^{2} \sin ^{2}\left(\frac{\delta}{2}\right)}
$$

and Equation 2.8 becomes

$$
I_{r}=I_{i} \frac{\left[2 r /\left(1-r^{2}\right)\right]^{2} \sin ^{2}\left(\frac{\delta}{2}\right)}{1+\left[2 r /\left(1-r^{2}\right)\right]^{2} \sin ^{2}\left(\frac{\delta}{2}\right)}
$$

Let us introduce a new quantity, coefficient of Finesse,

$$
F=\left(\frac{2 r}{1-r^{2}}\right)^{2}
$$

and introduce it in Equation 2.11 and Equation 2.12 respectively

$$
\begin{aligned}
& \frac{I_{t}}{I_{i}}=\frac{1}{1+F \sin ^{2}\left(\frac{\delta}{2}\right)} \\
& \frac{I_{r}}{I_{i}}=\frac{F \sin ^{2}\left(\frac{\delta}{2}\right)}{1+F \sin ^{2}\left(\frac{\delta}{2}\right)}
\end{aligned}
$$

Here, the term $\left[1+F \sin ^{2}\left(\frac{\delta}{2}\right)\right]^{-1}=\mathcal{A}(\theta)$, is known as Airy function. It represents the transmitted flux-density distribution and plotted in Figure 2.3 


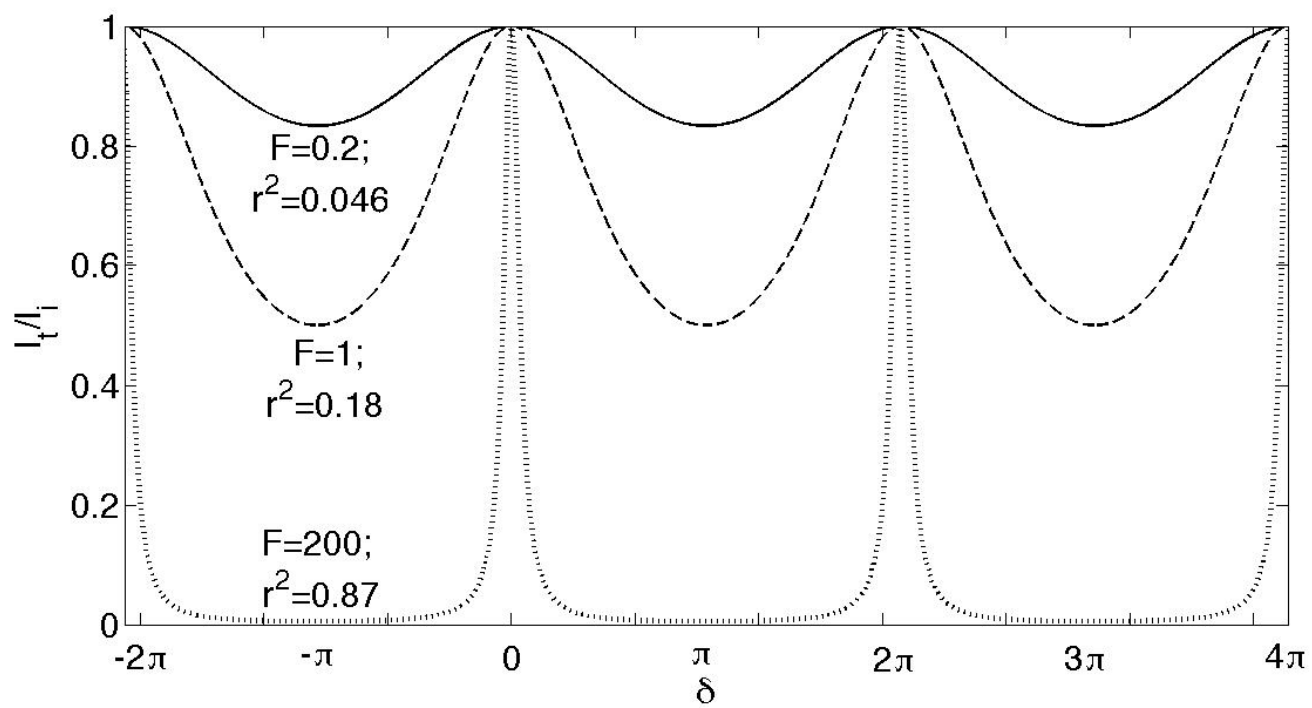

Figure 2.3 Transmission vs phase graph which follows Airy function

When $\delta / 2$ is $m \pi$, the Airy function is equal to unity for all values of $F$ and therefore $r$. When $r$ approaches 1 , the transmitted flux density is very small, except for the sharp spikes centered about the point $\delta / 2 m \pi$

The complementary function $[1-\mathcal{A}(\theta)]$ is plotted in Figure 2.4

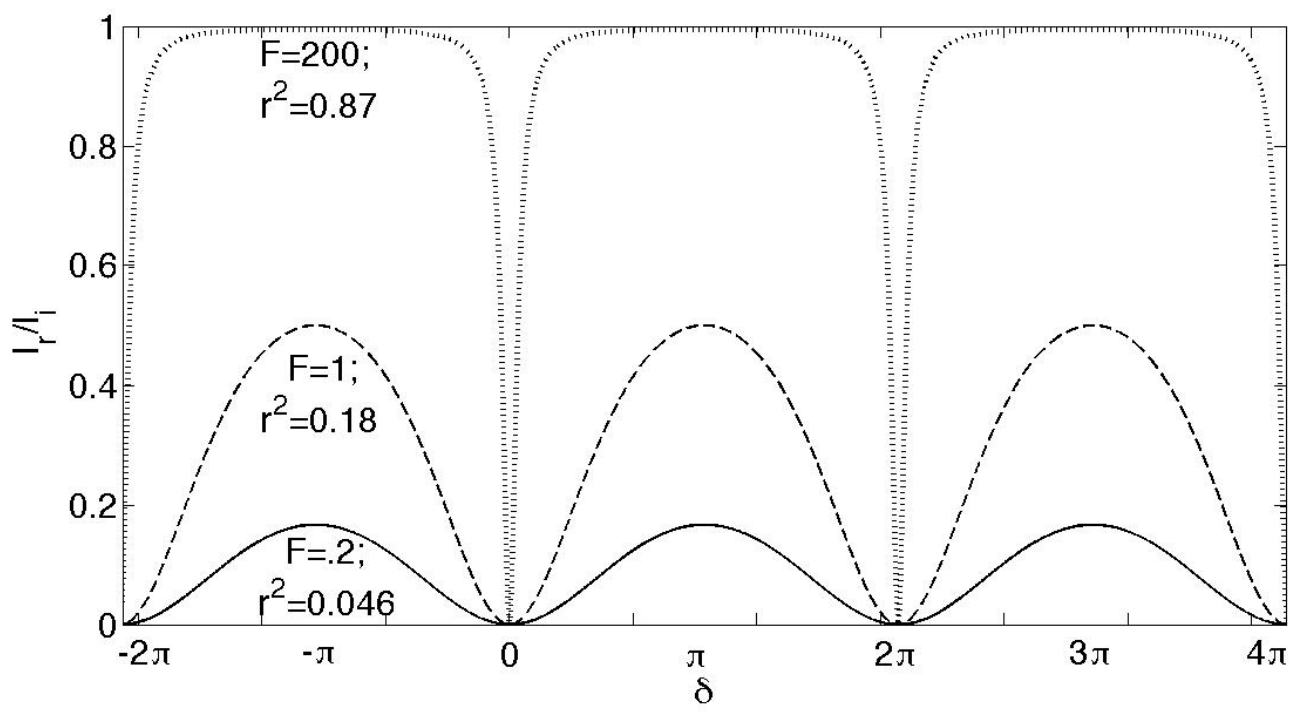

Figure 2.4 Reflection vs phase graph which follows (1-Airy function) 
On the parallel plate plane, the fringe due to transmitted light will consist of a series of narrow bright rings on dark background. The fringe due to reflected light would be narrow and dark on a uniformly bright background. Constant thickness, sharp and narrow fringes can be obtained by a light metal coating to the reflecting surfaces to produce multiple beam interference.

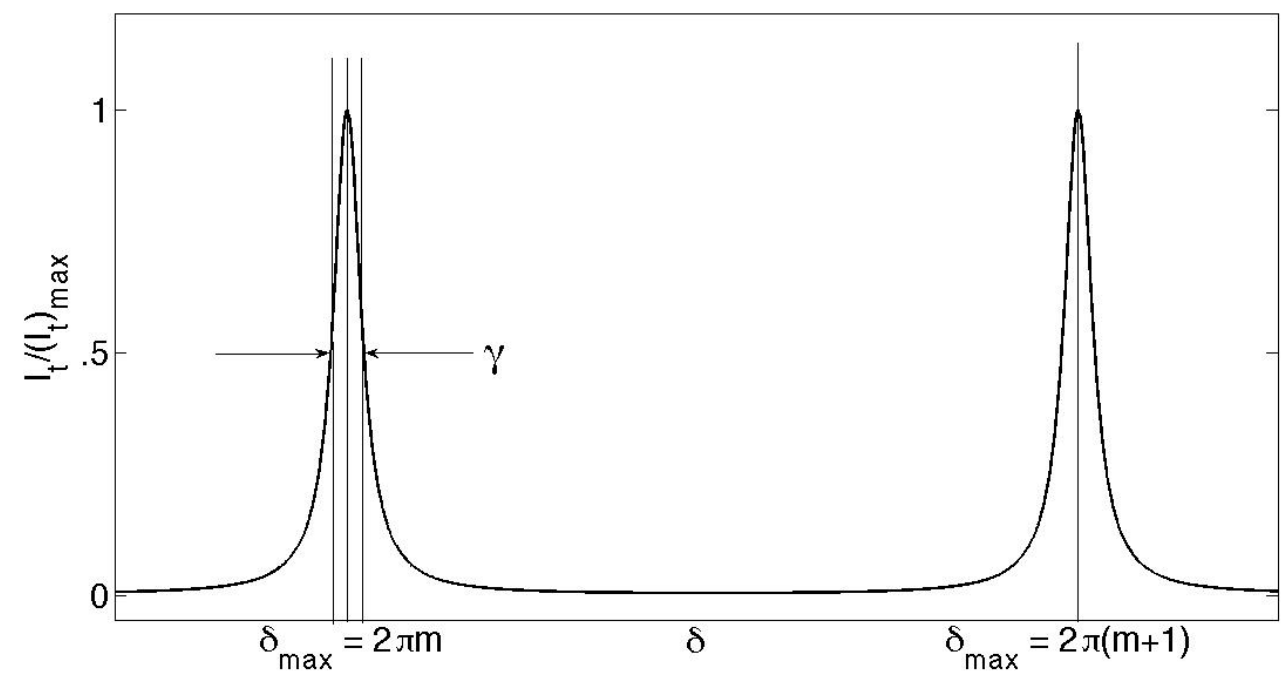

Figure 2.5 Fabry-Perot fringes plotted for $F=200$ and $R=0.87$

A measure of the sharpness of the fringes (how rapidly the irradiance drops off on either side of the maximum) is given by the half width $\gamma$. It is the width of the peak, in radians, when $I_{t}=\left(I_{t}\right)_{\max } / 2$ (Figure 2.5). Transmission peaks occur when the phase difference is $\delta_{\max }=2 \pi m$.

On same condition the irradiance will drop to half of its maximum value.

$$
\begin{gathered}
{[\mathcal{A}(\theta)=1 / 2] \text { and } \delta=\delta_{\max } \pm \delta_{1 / 2}} \\
\text { Now, } \mathcal{A}(\theta)=\left[1+F \sin ^{2}\left(\frac{\delta}{2}\right)\right]^{-1}=1 / 2
\end{gathered}
$$

Which follows,

$$
\delta_{1 / 2}=2 \sin ^{-1}\left(\frac{1}{\sqrt{F}}\right)
$$

Since $F$ is large, $\sin ^{-1}\left(\frac{1}{\sqrt{F}}\right) \approx\left(\frac{1}{\sqrt{F}}\right)$ and therefore half-width $\gamma=2 \delta_{1 / 2}$ becomes

$$
\gamma=\frac{4}{\sqrt{F}}
$$


We recall that, $F=4 R /(1-R)^{2}$, so that the larger $R$ is sharper transmission peaks.

The ratio of the separation of adjacent maxima to the half-width is known as Finesse defined as:

$$
\begin{aligned}
\mathcal{F} & =2 \pi / \gamma \\
\text { or, } \mathcal{F} & =\frac{\pi \sqrt{F}}{2}
\end{aligned}
$$

The Fineese $\mathcal{F}$ increases as the half width $\gamma$ decreases.

The distance between two resonance dip is called free spectral range (FSR) and expressed as,

$$
(\Delta v)_{F S R}=\frac{c}{2 n d}
$$

and the minimum resolvable bandwidth is

$$
(\Delta v)_{\min }=\frac{c}{\mathcal{F} 2 n d}
$$

The Finesse then defined as the ratio of free spectral range to the minimum resolvable bandwidth

$$
\frac{(\Delta v)_{F S R}}{(\Delta v)_{\min }}=\mathcal{F}
$$




\subsection{APPLICATION OF FP ETALON IN ULTRASOUND DETECTION}

A FP etalon can be used in ultrasound detection [8-14, 54-57]. When ultrasound is incident (Figure 2.6(a)) on a surface of a polymer etalon, its thickness is changed due to the pressure of the ultrasound wave (Figure 2.6(b)). A laser beam from the other side is used to detect the modulated thickness of the etalon. We consider a ray of laser incident onto other surface from the other side of this etalon. This laser ray will be reflected from the two parallel surfaces of the reduced thickness etalon (Figure 2.6(c)) (ignore the other successive reflections within the etalon). These two reflected rays have a certain phase difference, which varies with the thickness of the etalon. A measure of intensity of these two reflected rays can provide us the information of the etalon thickness as, under 'linearity' condition of ITF [8], there is a linear relationship between intensity and phase change which is proportional to etalon's thickness change. The etalon thickness change occurs according to the ultrasound pressure incident on it. Thus the FP etalon is used to detect an ultrasound pressure wave by measuring the reflected output intensity. Here the notable point is the etalon material, which has a thickness optimized to be sensitive to the ultrasound pressure. Normally polymer materials with gold coating on two sides are used to make an etalon.

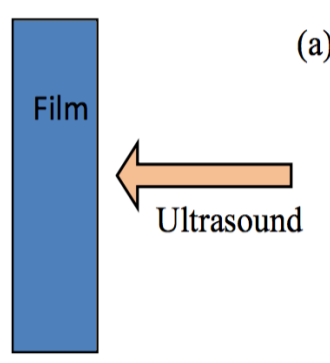

(a)

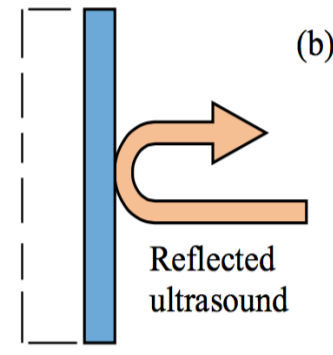

(b)

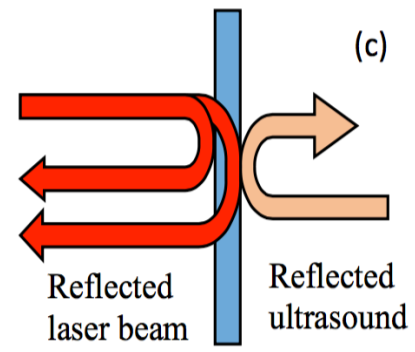

Figure 2.6 (a) Ultrasound is incident on a polymer etalon from its right side. (b) The thickness of the polymer etalon is modified due to the incident ultrasound pressure wave; and the ultrasound wave is reflected (c) A probing laser is incident from the left side of the etalon and later it is reflected from the two surfaces of the deformed etalon.

This technique has several benefits over traditional ultrasound detection methods [21]. Firstly, the active sensing area is defined by the spot size of the optical beam which can be reduced to few microns. Here no electrical connection is required. The detection bandwidth can be controlled by controlling the thickness of the material or choosing the appropriate material. 


\subsection{INTERFEROMETER TRANSFER FUNCTION (ITF)}

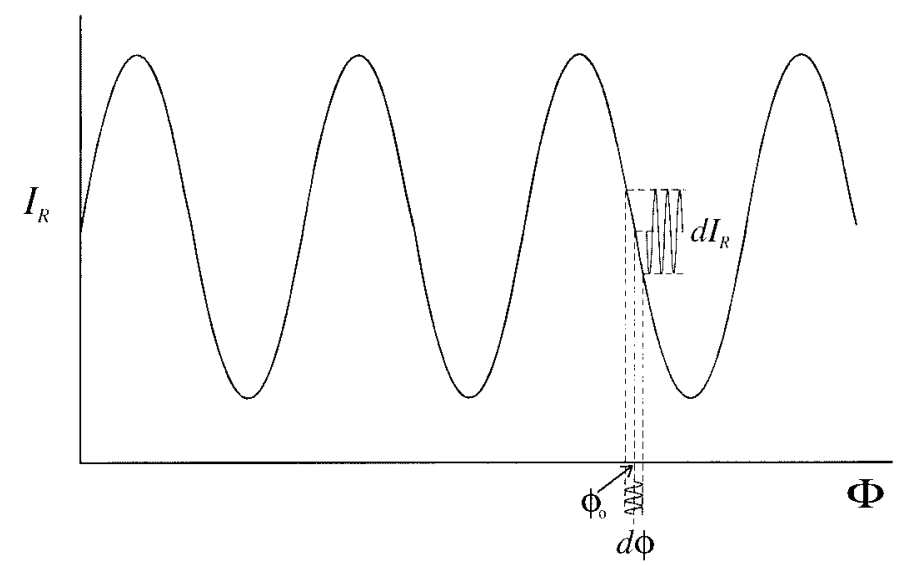

Figure 2.7 Interferometer transfer function (Here, $\left.\Phi=\phi_{0}+d \phi\right)[10]$

The relation between the reflected optical output $\left(I_{R}\right)$ of the interferometer and the optical phase $(\Phi)$ is termed as Interferometer Transfer Function (ITF) [10, 25-26]. This is a plot (Figure 2.7) of varying amplitude. The plot has linear portion, which signifies that the intensity change is proportional to the phase change within this linear range. For a reliable and useful experiment the laser wavelength should initially choose in a way so that the initial phase $\left(\phi_{0}\right)$ of it should stay in the most linear region. Then the intensity change is expected to be proportional to the phase change $(d \phi)$. Ultrasound detection using optical interferometer is a process where ultrasound pressure modulates the optical intensity through an ITF.

\subsection{SENSITIVITY OF THE FILM}

\subsubsection{Sensitivity Definition}

Let's consider a polymer sensing film [10-11] of thickness $l$ (Figure 2.8). The media on its two sides are different (media 1 and 2). The film has different acoustic impedance $Z$ than its two sides surrounding media with impedance $Z_{1}$ and $Z_{2}$. The film and its two sides acoustic reflection coefficients are $R, R_{1}$ and $R_{2}$. We consider the same film with two surfaces with very low reflectivity $r_{1}$ and $r_{2}\left(r_{1}, r_{2}<1 \%\right) . n, n_{1}, n_{2}$ are the refracting coefficients of the film and its two sides media 1 and 2. An optimal film should be 
sensitive acoustically as well as optically. In the following two sections we are going to find the acoustic sensitivity, optical sensitivity and the total sensitivity of the film.

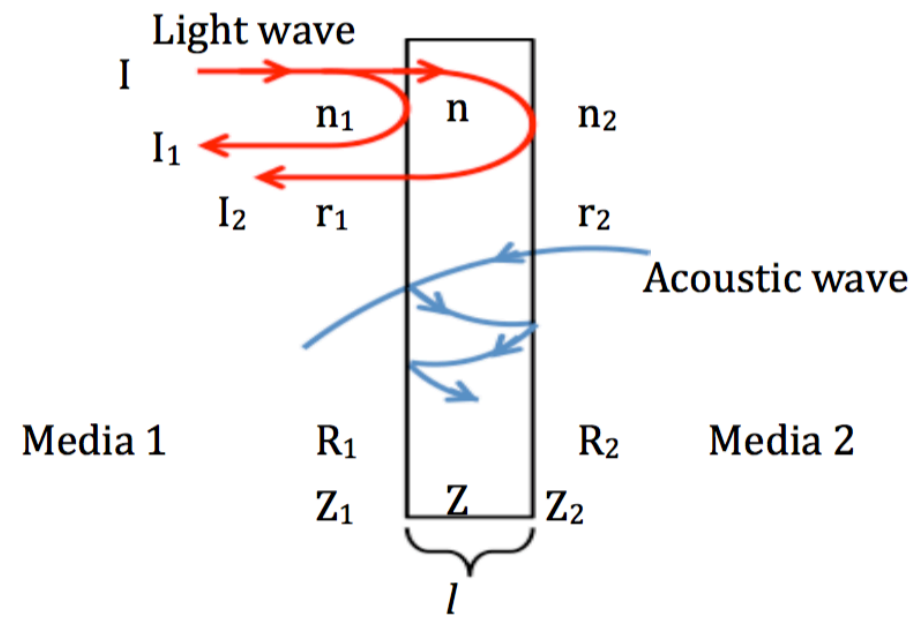

Figure 2.8 Diagram showing a polymer sensing film, where a laser light (shown by red line) incident and later reflected from the two surfaces of the film. The blue curves showing the successive internal reflection of acoustic wave inside the film.

Consider a light beam with intensity $I$ is incident on the film, it will be reflected from the film in turn. When ultrasound pressure $(P)$ is exerted on it, its thickness would be changed. Due to this thickness change, the reflected intensity would also be changed as the thickness change creates some phase modulation. The change of optical intensity $(d I)$ with the change of pressure $(d P)$ is a measure of the sensitivity for the film. When a very low pressure on the film makes a larger optical intensity variation, it is most sensitive to small pressures. The change of optical intensity $(d I)$ with the change of pressure $(d P)$, written as $\frac{d I}{d P}$ can be expressed in terms of phase change $(d \phi)$ in the following way:

$$
\frac{d I}{d P}=\frac{d I}{d \phi} \frac{d \phi}{d P}
$$

Here $\left(\frac{d I}{d P}\right)$ is total sensitivity of the film. $\left(\frac{d I}{d \varphi}\right)$ is optical sensitivity and $\left(\frac{d \phi}{d P}\right)$ is called acoustic sensitivity. We can write,

Total Sensitivity $=($ Optical Sensitivity $) \times($ Acoustic Sensitivity) $\quad$ Equation 2.23 Both the optical and acoustic phase sensitivities have their own mathematical expressions and they are discussed below. 


\subsubsection{Acoustic Sensitivity}

Acoustic sensitivity is defined as the magnitude of the optical phase shift produced per unit acoustic pressure [11]. For the initial ultrasound pressure amplitude $P_{0}$, it is specified as $\frac{d \phi}{P_{0}}$.

When a sinusoidal acoustic wave normally incident on the film, there will be multiple acoustic reflections within the film due to the different acoustic impedance with the surrounding media (shown in Figure 2.8). The thickness will be changed by the strain of the acoustic wave. We consider the change of the thickness is $d l$, which create a phase shift $d \phi$.

$$
\text { Now, } d \phi=\frac{4 \pi n d l}{\lambda}
$$

Here $\lambda$ is the wavelength of laser source. The thickness change of the film is given by

$$
d l=\int_{0}^{l} \frac{P_{T}(x, t)}{E} d x
$$

Here $\mathrm{E}$ is the Youngs modulus of the polymer film. $P_{T}(x, t)$ is the spatial distribution of pressure across the thickness of the film and is the sum of the component of the incident acoustic wave that is transmitted into the film $P_{1}$, and subsequent acoustic reflections $P_{2}, P_{3} \ldots \ldots, P_{n}$ at the boundaries of the film.

We consider the sinusoidal acoustic wave (which has pressure amplitude $P_{0}$ ) has angular acoustic frequency $\omega$ and it is travelling in negative $x$ direction Then $P_{T}$ will be,

$$
P_{T}=P_{0} T \sum_{i=0}^{(N-1) / 2}\left\{R_{1}^{i} R_{2}^{i} \sin [\omega t-\mathcal{K}(2 l i-x)]+R_{1}^{i+1} R_{2}^{i} \sin [\omega t-(2 l i+x)]\right\}
$$

Equation 2.26

Here, $\mathcal{K}=\frac{2 \pi}{\lambda_{a}} ; \lambda_{a}=$ Acoustic wavelength. $T$ is the pressure amplitude transmission coefficient. $R_{1}$ and $R_{2}$ are the pressure amplitude reflection coefficients at the two surfaces of the film, and

$$
\begin{aligned}
& T=\frac{2 Z}{Z+Z_{2}} \\
& R_{1}=\frac{Z_{1}-Z}{Z+Z_{1}} \\
& R_{2}=\frac{Z_{2}-Z}{Z+Z_{2}}
\end{aligned}
$$


Substituting $P_{T}$ value in Equation 2.25, we get

$$
d l=\frac{P_{0} T}{E \kappa} \sum_{i=1}^{N+2} \psi_{i} \cos \left(\omega t+\xi_{i}\right)
$$

Here $\xi$ is phase for the cos function.

Which reduces to

$$
d l=\frac{P_{0} T}{E \kappa} \psi \cos (\omega t+\xi)
$$

where the amplitude is $\psi=\left[\left(\sum_{i=1}^{N+2} \psi_{i} \cos \xi_{i}\right)^{2}+\left(\sum_{i=1}^{N+2} \psi_{i} \sin \xi_{i}\right)^{2}\right]^{1 / 2}$

Now the thickness of the film becomes

$$
d l_{0}=\frac{P_{0} T}{E \kappa} \psi
$$

For modeling we can consider only 5 multiple reflection. Then $\psi$ would be written as $\psi_{5}$

$$
\text { So, } d l_{0}=\frac{P_{0} T}{E \kappa} \psi_{5}
$$

Here

$$
\begin{aligned}
\psi_{5}^{2}=\left[\left(1-R_{1}\right.\right. & +\left(R_{1}-R_{2} R_{1}-1\right) \cos \kappa l+\left(R_{2} R_{1}-R_{2} R_{1}^{2}\right) \cos 2 \kappa l \\
& +\left(R_{2} R_{1}{ }^{2}-R_{2}{ }^{2} R_{1}^{2}\right) \cos 3 \kappa l+\left(R_{2}{ }^{2} R_{1}{ }^{2}-R_{2}{ }^{2} R_{1}^{3}\right) \cos 4 \kappa l \\
& \left.+R_{2}^{2} R_{1}^{3} \cos 5 \mathcal{K} l\right]^{2} \\
& +\left[\left(R_{2} R_{1}-R_{1}-1\right) \sin \kappa l-\left(R_{2} R_{1}-R_{2} R_{1}^{2}\right) \sin 2 \kappa l-\left(R_{2} R_{1}^{2}\right.\right. \\
& \left.\left.-R_{2}^{2} R_{1}^{2}\right) \sin 3 \kappa l-\left(R_{2}^{2} R_{1}^{2}-R_{1}^{3}\right) \sin 4 \kappa l-R_{2}^{2} R_{1}^{3} \sin 5 k l\right]^{2}
\end{aligned}
$$

Equation 2.34

The phase modulation per unit acoustic pressure then becomes

$$
\frac{d \phi}{P_{0}}=\frac{4 \pi n}{\lambda} \frac{T}{E_{k}} \psi_{5}
$$

Which is called the acoustic sensitivity.

\subsubsection{Optical Sensitivity}

Let $I_{1}, I_{2}$ be the intensities of the two reflected rays [10] from the two surfaces of the film (Figure 2.8) then the resultant intensity $I_{0}$ would be the superposition of these two intensities.

$$
I_{0}=I_{1}+I_{2}+2 \sqrt{I_{1} I_{2}} \cos \Phi
$$

Here $\Phi$ is the sum of initial phase $\phi$ and the changed amount of phase $d \phi$, which is very small. 
We obtained $d I_{0}$ from Equation 2.36

$$
d I_{0}=-2 \sqrt{I_{1} I_{2}} d \phi
$$

Equation 2.37

$I_{1}$ and $I_{2}$ can be written in terms of the incident intensity $I$ and the Fresnel reflection coefficient $r_{1}$ and $r_{2}$, defined as $I_{1}=I r_{1}$ and $I_{2}=I\left(1-r_{1}\right)^{2} r_{2}$, Where $r_{1}=\left(\frac{n-n_{1}}{n+n_{1}}\right)^{2}, r_{2}=$ $\left(\frac{n-n_{2}}{n+n_{2}}\right)^{2}$

The phase modulation per unit phase shift is now

$$
\frac{d I_{0}}{d \phi}=-2 I\left(1-r_{1}\right) \sqrt{r_{1} r_{2}}
$$

Equation 2.38

Which is called optical phase sensitivity.

\subsubsection{Practical Sensitivity Parameters}

In practice the total sensitivity depends on [10-11] optimal values of surface reflectivity $\left(r_{1}\right)$, low Youngs modulus of the film, increased laser power. Also, films adequate uniformity, surface finis and optical clarity are responsible for a film's sensitivity. 


\section{Chapter 3 MATERIALS AND METHODS}

\subsection{FABRICATION OF ETALON}

It is very important to choose appropriate material to make an etalon for our work, as an etalon has to be sensitive to low pressures. We planned to make an etalon with 5 layers (Figure 3.1). The layers were: a glass substrate, gold, a polymer, gold, and a final layer of polymer for water protection. The metal gold layer was used in each side of the polymer film to improve the reflectivity of its two surfaces.

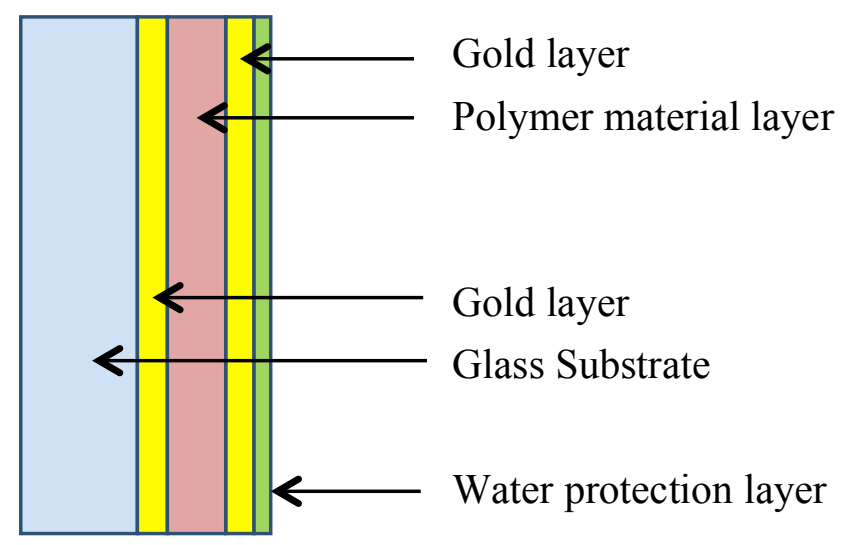

Figure 3.1 Schematic diagram for the etalon

We planned to make the etalon with two materials. The materials were 'SU8' [28-30] and 'Parylene-C' [31-32]. These are two special types of polymer. We choose these two materials because our main purpose was making a uniform thin film. Moreover, attempts to make an interferometer were successfully completed using these two materials [9, 21].

The material SU8 consists of chemically amplified, epoxy based negative photoresist (Youngs modulus (2.92-4.95) GPa, index of refraction 1.596). It is highly functional and optically transparent. Cured films are very resistant to solvents, acids and bases. SU8 has excellent thermal and mechanical stability. Using spin-coating method, it is possible to make a very thin film ranging from few hundred nanometers to hundreds of microns. 
Parylene-C is the trade name for a variety of chemical vapor deposited polymers (Youngs modulus $2.9 \mathrm{GPa}$, index of refraction 1.639) used as moisture and dielectric barriers. Its excellent moisture barrier property is used for coating printed circuit boards. It is bioaccepted material for which it is widely used in medical sectors to make medical devices. Besides, it has a low di-electric constant, optical clarity, surface conformability, low-cost and other advantages. Parylene-C can be deposited using chemical vapor deposition process, which has more precise thickness and uniformity control over the method.

We deposited both films on a glass substrate. The process 'Substrate Preparation' was the first step and was common for preparing both etalons. After this, the second common step was 'Gold Coating' on the cleaned substrate. Each of the cleaned glass substrate was coated (coated on the top surface of the glass slide only) with a particular thickness of gold using a sputtering method. On this gold-coated glass slide, the polymer film materials were deposited later to make the interferometer.

\subsubsection{Substrate Preparation}

A glass slide was used as our substrate to make our FP etalons. The substrate [33] dimensions were: $\left(75 \mathrm{~mm} * 25 \mathrm{~mm}^{*} 1 \mathrm{~mm}\right)$. The glass substrates were first cleaned with Piranha solution, which is a mixture of Hydrogen per oxide and Sulphuric acid [34]. Then the substrates were cleaned with Ethanol and Acetone. Then they were dipped in deionized water. After that, they were dried by Nitrogen blow. The clean substrates were prepared and stored carefully for our next process, and did not touch them directly with our hand.

\subsubsection{Gold Coating Method}

On the top surface of the cleaned glass sample, a layer of gold of particular thickness was made. The sputtering method was used for the gold coating. 

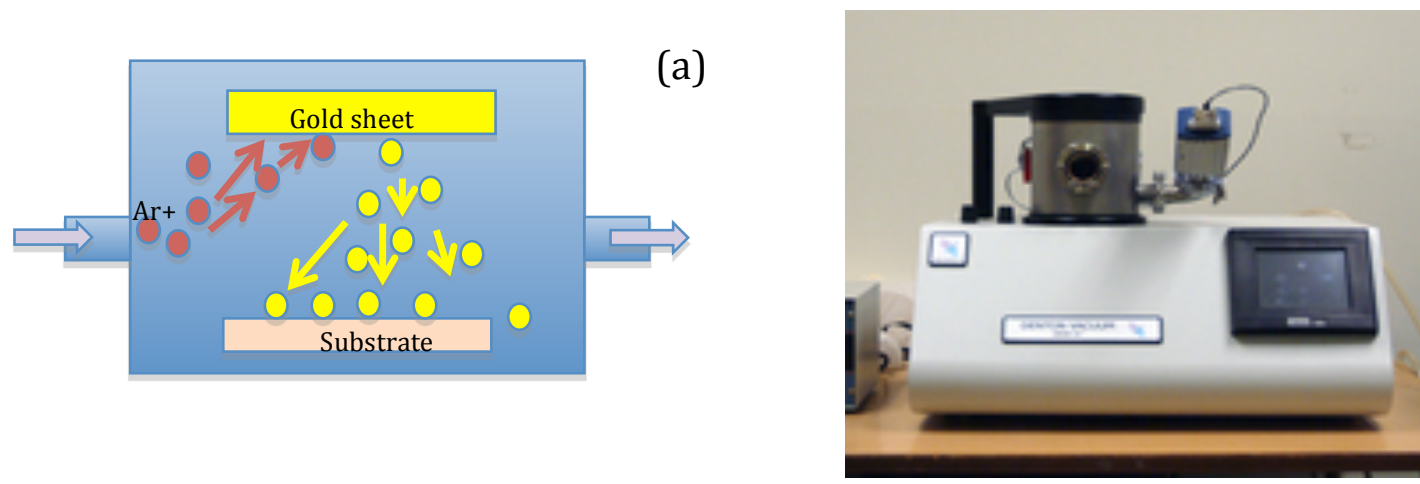

(b)

Figure 3.2 (a) Schematic diagram for a sputtering process (b) Sputter device [44]

In sputtering process (Figure 3.2(a)), a target made of the material to be deposited (gold in our case) is bombarded by high-energy ions $\left(\mathrm{Ar}^{+}\right)$that dislodge atoms of the target. The dislodged atoms have a range of kinetic energies; some of these atoms are incident onto the substrate and make a coating of the target material. The overall process is completed in a vacuum chamber and in a very low pressure.

We used a sputtering machine shown in Figure 3.2(b) (MODEL: Denton Vacuum Desk IV) and gold was used as the target. The chamber pressure was $\sim 5 \times 10^{-6}$ Torr. The 2 inches diameter metal stage in the chamber where we put our glass samples was stationary. For our sputtering device, the thickness of the gold layer increases linearly with time. Initially, we were unable to be certain of the thickness of the coated gold. Therefore, we made different thickness of gold by using different gold deposition times. For our experiment we used deposition times of a minimum of 10 seconds to a maximum 350 seconds. Later we measured the relation of the thickness of the gold as a function of the gold coating time. The measurement process is described at the end of this chapter. 


\subsubsection{SU8 Etalon Making Process}

We choose the spin coating method to prepare the SU8 film interferometer. We used a spinner/ spin coater device to do this deposition.

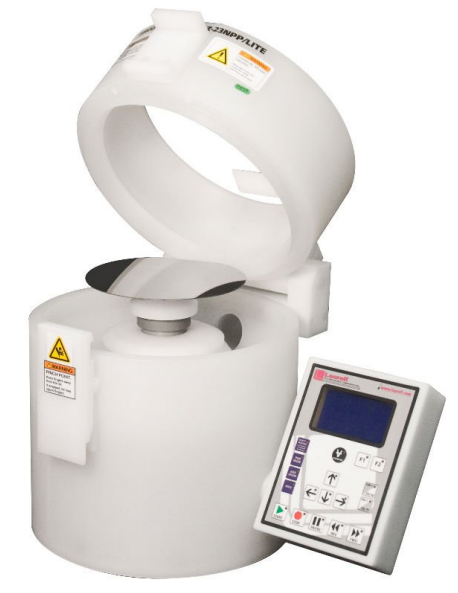

Figure 3.3 Spin coater device [43]

Spin coater device (Figure 3.3) is a bucket-like device, which has an opening of vacuum pipe at the center of the 'bucket'. On this vacuum pipe, the substrate is horizontally placed. The central portion rotates with a high speed. The rotation speed (acceleration), time of rotation, rotation stages can set by an adjacent device. The spinner device has a lid, which must be closed during its operation.

When the vacuum pipe is turned on with the substrate on it, the substrate becomes tightly adjusted to the vacuum pipe so that the substrate does not drop from the central pipe during its high-speed rotation. After the substrate adjustment, few drops of raw SU8 is poured on the substrate. The substrate then allowed to rotate according to the pre-set acceleration (ACL) and time of rotation. Sometimes there are more than one stage settings with different time of rotation and acceleration. A layer of thin film of a particular thickness is formed on the substrate due to the rotation of the substrate. The thickness of SU8 depends on the speed (acceleration) and time of rotation. According to the material availability, we choose SU8-2000 photoresist [30] and aimed to make a $8 \mu \mathrm{m}$ thick SU8 film on gold coated glass substrate. 
The steps of SU8 film formation were as follows: [36]

1. We kept the cleaned glass slide on the vacuum pipe (rotation stage) where gold-coated portion was facing up. Then we turned on the vacuum pipe so that the slide was securely attached to the vacuum pipe.

2. We dispensed few drops of liquid raw SU8-2000 in the middle of the slide.

3. The spinner then span at $500 \mathrm{rpm}$ for 5 seconds (ACL: 2), then ramped to $1500 \mathrm{rpm}$ for 30 seconds (ACL: 7). This process made a layer of $\sim 8 \mu \mathrm{m}$ thick SU8 film on a gold coated glass slide, which was still in its raw state.

4. To make the film rigid, we 'soft baked' it for $2 \mathrm{~min}$ at $65^{\circ} \mathrm{C}$, then increased the hotplate temperature to $100^{\circ} \mathrm{C}$. Once the temperature reached to $95^{\circ} \mathrm{C}$, we baked it 5 more minutes.

5. We exposed the slide to UV-light for 6.3 seconds.

6. We did the post exposure bake (PEB) for $3 \mathrm{~min}$ at $65^{\circ} \mathrm{C}$, then increased the hot plate temperature to $100^{\circ} \mathrm{C}$. Once the temperature reaches to $95^{\circ} \mathrm{C}$, we baked it 7 more minutes.

The above steps gave us approximately an $8 \mu \mathrm{m}$ thick SU8 film on the gold- coated glass. After finishing this process, another layer of gold was layered on top of the film following the procedure mentioned above. It was then returned again to the lab for another $8 \mu \mathrm{m}$ thick SU8 layer on the film following the same steps of SU8 film formation mentioned above. This layer was actually a layer to protect the film from water. When these were completed, silicone gel was used around its border to make the multilayer film leak-proof.

We made 3 SU8 film etalons with 3 different gold layer thicknesses. The gold coating times for the 3 different films were: $92 \mathrm{~s}, 138 \mathrm{~s}, 246 \mathrm{~s}$. 


\subsubsection{Parylene-C Etalon Making Process}

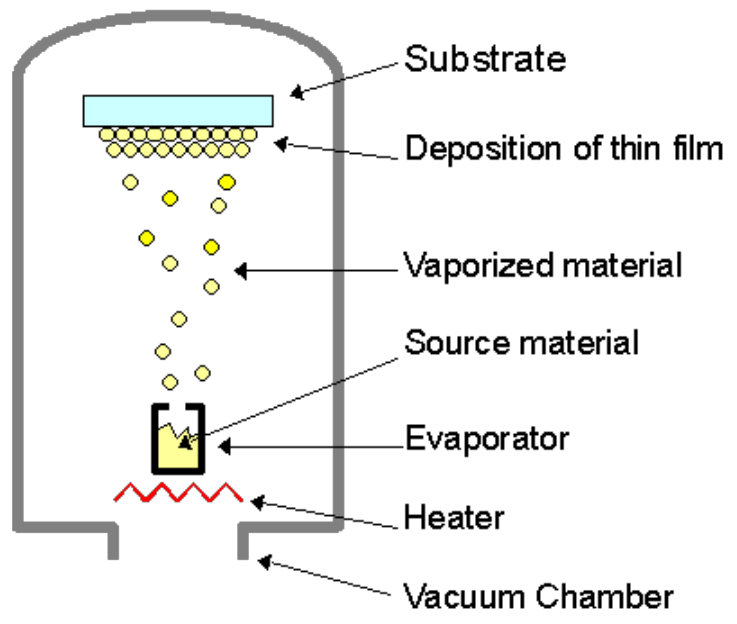

(a)

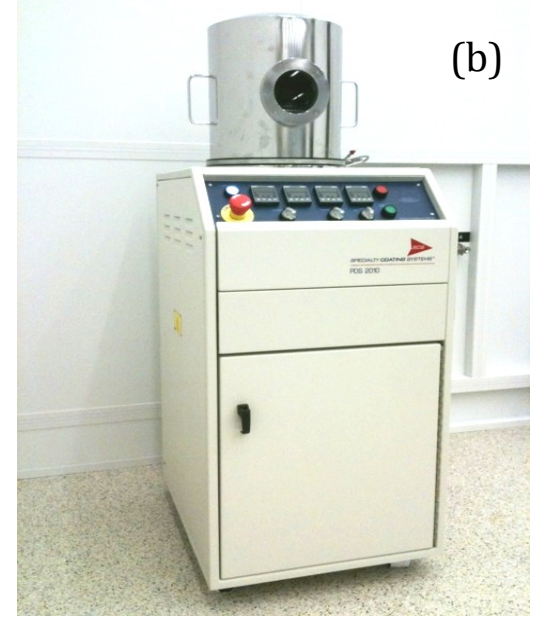

Figure 3.4 (a) Schematic diagram of vacuum evaporation technique [41] (b) Parylene coater device [42]

Parylene film was deposited by vacuum evaporation technique (Figure 3.4(a)). In this technique, certain amount of raw Parylene beads was kept on a tray (evaporation boat) of a vacuum chamber. The substrates on which thin films would be formed were kept attached to the chamber top facing the surface to be film deposited down. Then the chamber was kept in a particular temperature for a certain period of time. With time a thin, transparent layer of Parylene film was deposited on the substrates. The thickness depends on the mass of the raw beads, chamber temperature, and film formation time. This process was a slow process (typical time $7 \mathrm{hr}-20 \mathrm{hr}$ ), and for its slow formation, it makes more uniform film than other quicker method like spinning.

We used a Parylene coater device, shown in Figure 3.4 (b), (MODEL: SCS Lab coater 2(PDS2000)) to make our Parylene films [37]. As first step of Parylene-C film formation, we cleaned the vacuum chamber. We turned on the vacuum chamber and wait until the pressure gauge stops increasing the readout number. Then we loaded the gold-coated glass samples (all together) in the deposition chamber, and closed the heavy lid. We opened the vaporizer door and inserted $15 \mathrm{~g}$ of Parylene-C dimer and spread it by a spatula. After doing this we closed the vaporizer door and latched it. We closed the front panel. We replaced the cold trap and started the vacuum pump. We set all operating parameters to start the process. The process stared. We left the started system for 7 hours. 
After 7 hours the sample were unloaded and carefully kept them scratch free. Then the samples were sent for another coating of gold on top of the Parylene-C films.

In this process we made 12 samples with 12 different thicknesses of gold coating. The gold coating time for the 12 samples were: 20s, 40s, 60s, 80s, 100s, 120s, 140s, $160 \mathrm{~s}, 180 \mathrm{~s}, 200 \mathrm{~s}, 220 \mathrm{~s}, 246 \mathrm{~s}$. Among these 12 samples, 3 samples were constructed with a $8 \mu \mathrm{m}$ water protection layer of SU8 by the spinning method (mentioned above). All the 12 samples finally made leak-proof around their boundary by using Silicon gel.

\subsection{OPTICAL CHARACTERIZATION OF PREPARED ETALONS}

We characterized all the newly made films optically by inserting them inside a spectrometer and observed their characteristics in transmission mood. A spectrometer is a device that measures the optical properties of any material over a range of wavelengths. It uses a lamp as a compound light source. When a spectrometer is switched on, the compound light is incident upon a grating. The grating acts as a prism which separates the light into its component wavelengths. The separated light of single wavelength then passes through a slit (light of only one wavelength emerges from the grating passes this slit). Beyond this slit, the sample is placed. The single wavelength light then passes through the sample. The reduced intensity light is then captured by a detector which records the intensity of the light. The grating of this system continuously rotates and the light wavelength that passes through the slit changes with the grating rotation; which changes the intensity information that reaches the detector through the sample. Using this process we record transmission spectra of material. For this work, a transmission

spectrum is capable to provide the information of film's quality (reflectivity, Finesse, uniformity) and thickness of the film. 


\subsection{EXPERIMENTAL SET-UP FOR OPTICAL DETECTION OF ULTRASOUND}

\subsubsection{Experimental Set-up and Devices}

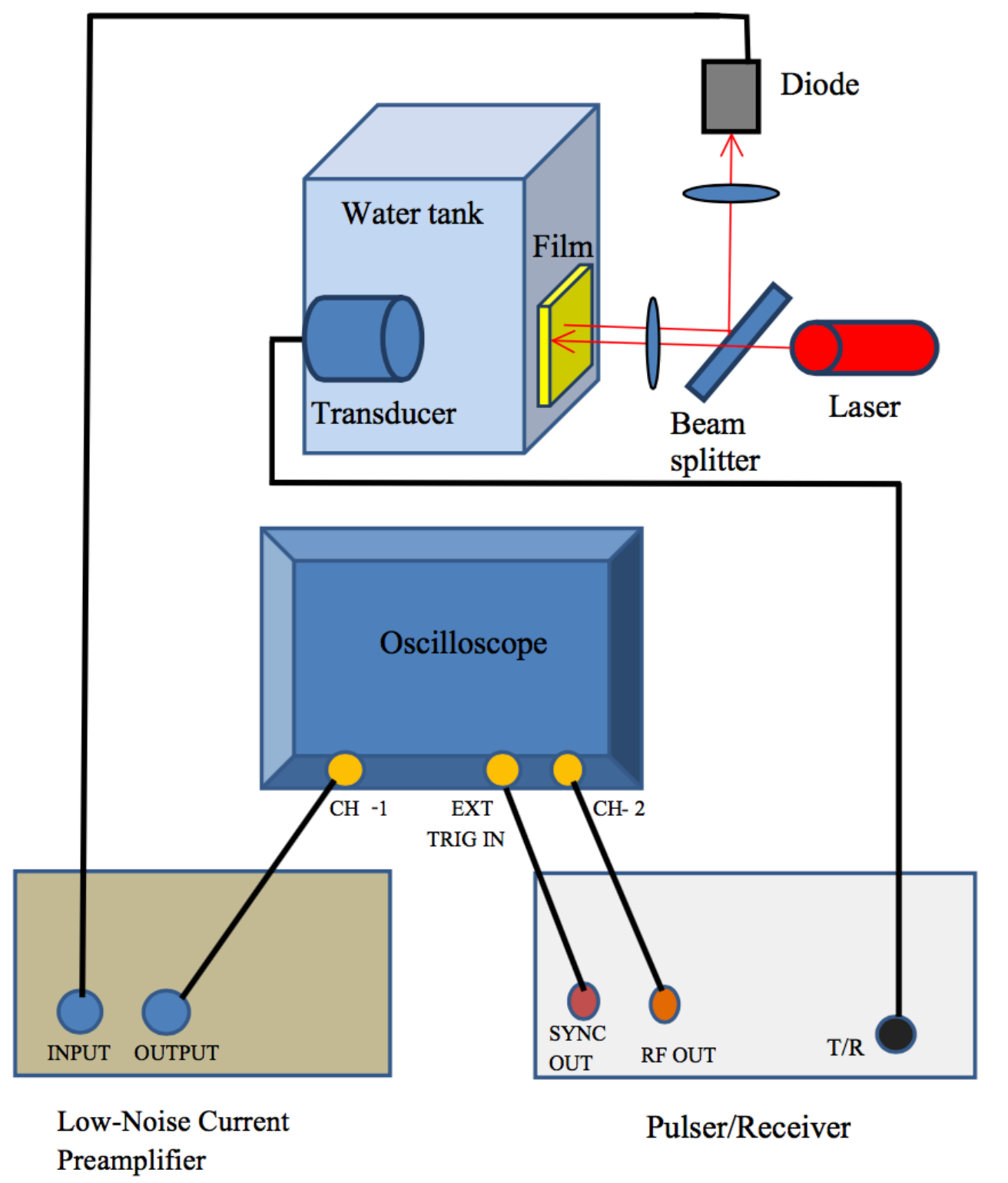

Figure 3.5 Schematic diagram of the full experimental set-up 
A schematic diagram for our full experimental arrangement is shown in Figure 3.5. A transparent acrylic tank with dimension $\sim(12$ "*12"*12”) was filled with water. A $1 \mathrm{MHz}$ transducer was positioned horizontally in the water tank and was placed so that its focal point was on the wall of the tank. The etalon film was kept at this location (inside the water tank). A pulser/receiver was connected to the transducer to drive it. A laser was incident onto the film from outside the tank. The laser beam goes to the film through a beam-splitter and the reflected ray comes in the same way, incident on the beam-splitter again. The beam-splitter then passed a portion of the reflected ray to a diode which was connected to a low noise current pre-amplifier. There were another synchronizing connection between the oscilloscope and the pulser receiver device. The pre-amplifier and pulser/receiver, both were connected to two channels of the oscilloscope to see their signal output.

The following table describing the model of the devices that we used in our experiment

Table 3.1 Description of the devices and material models

\begin{tabular}{|l|l|}
\hline \multicolumn{1}{|c|}{ Device Name } & \multicolumn{1}{c|}{ Company name / Model } \\
\hline Ultrasonic pulser receiver & Olympus ultrasonic pulser receiver 5077PR \\
\hline Low noise pre-amplifier & Stanford Research System SR570 \\
\hline Laser-1 & Metrologic Neon Laser (5mW red laser) \\
\hline Laser-2 & $\begin{array}{l}\text { Creaton Inc. LASER-001054 (5mW red laser with } \\
\text { wavelength 650 nm) }\end{array}$ \\
\hline Diode & Vishay semiconductors BPW34 \\
\hline Spectrometer & Shimadzu UV-3600 \\
\hline Optical glass slide & Fisherbrand \\
\hline Hydrophone & ONDA- HNP-0400, S/N: 1002 (Needle hydrophone) \\
\hline
\end{tabular}



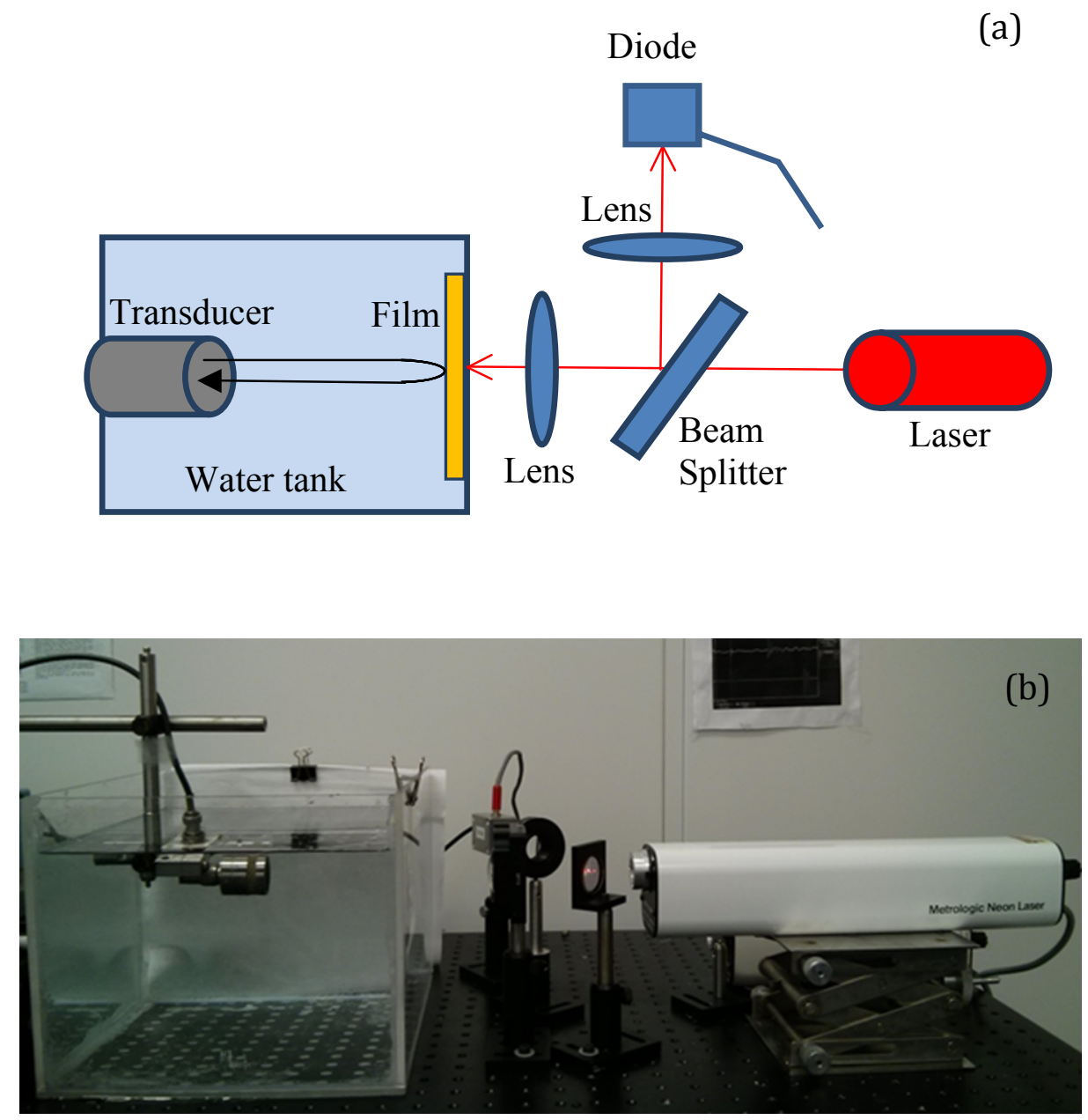

Figure 3.6 (a) Schematic diagram (b) A picture of the major portion of our experimental set up.

The major portion of Figure 3.5 is shown in Figure 3.6. When the transducer is excited, it emits a pulse. The pulse travels to the film and is reflection off the surface of the film. The reflected signal is then received by the same transducer. The acoustic signal travels two times the distance between transducer and the film surface. In our experiment we detected the acoustic signal optically and instantly at the time when the acoustic wave is incident upon the film surface. The optical signal was always expected to be located halfway between the initial excitation pulse and first reflection pulse.

We used A 5mW red neon laser (Laser-1 of Table 3.1) as our optical source. The diode we were using here was a $7.5 \mathrm{~mm}^{2},\left(\mathrm{NEP} 4^{*} 10^{-4} \mathrm{~W} / \sqrt{\mathrm{Hz}}\right)$ diode. Initially we used a 1 
$\mathrm{MHz}$ focused transducer with a focal length of 4 inches and with a diameter 1 inch. Inside the acrylic water tank, we set the $1 \mathrm{MHz}$ transducer a distance away from the tank wall which was equal to its focal distance (focal point=a point in water where the reflected acoustic signal found strongest), so that the first ultrasound echo was expected to be located at a distance that is twice the focal length of the transducer in the measured time-domain signals.

\subsubsection{Signal Characteristics Measurement}

We followed the procedure described below to calculate various parameters from the measured optical signal

a) Frequency measurement: We measured the frequency of a signal manually by this way:

$$
\text { Signal frequency }=1 /(2 * \text { peak-to-peak distance along } x \text {-axis }) \quad \text { Equation } 3.1
$$

b) SNR measurement: To calculate the SNR of each optical signal, we first measured the 'Signal's height manually. For Noise measurement we measured the standard deviation of the left side portion of the optical 'Signal' in matlab. Then the obtained 'Signal' was divided by the 'Noise' value

c) Gain, Averaging, Pulser voltage: The pulser receiver device had two knobs that showed the Gain and Pulser voltages of any ultrasound signal. The averaging of any optical signal was shown on the digital oscilloscope monitor. 


\subsection{OPTICAL DETECTION OF DIFFERENT FREQUENCY ULTRASOUND}

In the following table we are introducing a description of the characteristics of the transducers used in our experiment.

Table 3.2: Description of the transducers

\begin{tabular}{|l|l|l|l|l|l|}
\hline $\begin{array}{c}\text { Transducer } \\
\text { Serial Number }\end{array}$ & $\begin{array}{c}\text { Transducer's } \\
\text { original } \\
\text { frequency } \\
(\mathrm{MHz})\end{array}$ & $\begin{array}{c}\text { Focal length, } \\
\mathrm{f}(\mathrm{inch})\end{array}$ & $\begin{array}{c}\text { Transducer } \\
\text { diameter, D } \\
\text { (inch) }\end{array}$ & $\begin{array}{c}\text { f-number } \\
(\mathrm{f} / \mathrm{D})\end{array}$ & \multicolumn{1}{|c|}{$\begin{array}{c}\text { Manufacture Co. } \\
\text { description }\end{array}$} \\
\hline 1 & 1.00 & 4.00 & 1.00 & 4.00 & $\begin{array}{l}\text { AEROTECH, ALPHA } \\
1.0 \text { MHz } 1,4.0 \text { IN FOCUS } \\
2905459,019518\end{array}$ \\
\hline 2 & 1.00 & 2.00 & 1.00 & 2.00 & $\begin{array}{l}\text { ILO 108HR, 1MHz/1.0 } \\
\text { SF }=2.0,10 \mathrm{DO} 65\end{array}$ \\
\hline 3 & 0.50 & 1.32 & 1.00 & 1.32 & $\begin{array}{l}\text { 0.5MHz, Transducer } \\
\text { ILO .508HP, (10D068) }\end{array}$ \\
\hline
\end{tabular}

\subsection{OPTICAL SIGNAL STRENGTH VARIATION WITH FILM PRESSURE}

In this experiment our goal was to observe the optical signal's amplitude variation with pressure (ultrasound pressure that is exerted on the film in the water-tank). This was done in three steps. For example, consider a, b, c are three parameters. By plotting (a vs. c) and (b vs. c), we can plot (a vs. b).

As the first two steps of our measurement, we plotted the 'Optical signal amplitude variation with pulser voltage' and as the second step measurement, we plotted 'Pressure variation with pulser voltage' from measurements made with a calibrated hydrophone. Then we finally plotted 'Optical signal amplitude variation with pressure'.

The pulser/receiver device that we used had four options to control the signal strength by controlling the current fed to the device. The four options were $100 \mathrm{~V}, 200 \mathrm{~V}, 300 \mathrm{~V}$, and $400 \mathrm{~V}$. We also used an attenuator between the connection of transducer and the pulser/receiver. An attenuator of magnitude 10 divides the pulser/receiver output voltage by 10 . So, by using an attenuator of magnitude 10 in our circuit, we made 4 more differed output voltages of the pulser/ receiver device. Using a $1 \mathrm{MHz}$ transducer, we observed and recorded the optical signal amplitude for all these 8 voltages (using and not using the attenuator in the circuit). We then plotted 'Optical signal amplitude variation with pulser voltage'. 


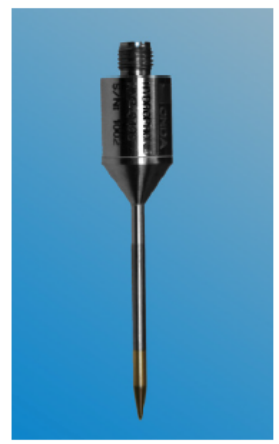

Figure 3.7 Hydrophone [49]

Our second step measurement was an experiment with hydrophone (Figure 3.7). At the transducer's focal point (inside water) we placed a hydrophone. We observed and recorded the pressure values (with the help of some calibrated data) measured by the hydrophone sensor tip. Using these data, we plotted 'Pressure variation with pulser voltage'.

Finally, using the data of first two plots mentioned above, we plotted 'Optical signal amplitude variation with pressure'.

\subsection{EXPERIMENTAL SET-UP FOR OBLIQUE LIGHT INCIDENCE SYSTEM}

Here we used the same set up as Figure 3.5 but here additionally we made our light incident system with variable angles instead of a zero degree angle (Figure 3.8). We used a goniometer on the optical bench to measure the incident angle. As we were not using the zero degree incident angle, beam-splitter was unnecessary here. The reflected ray was directly captured by the diode. The diode was on a stand, which was moveable on optical bench. Here we used a comparatively small size laser (Laser-2 of Table 3.1) for our set up convenience, a $5 \mathrm{~mW}$ red laser with $650 \mathrm{~nm}$ wavelength. Our purpose was to make the incidence angle range from 0.25 degree to 89.75 degree, but for our instrumental condition, the range could set only (8-82) degree. 


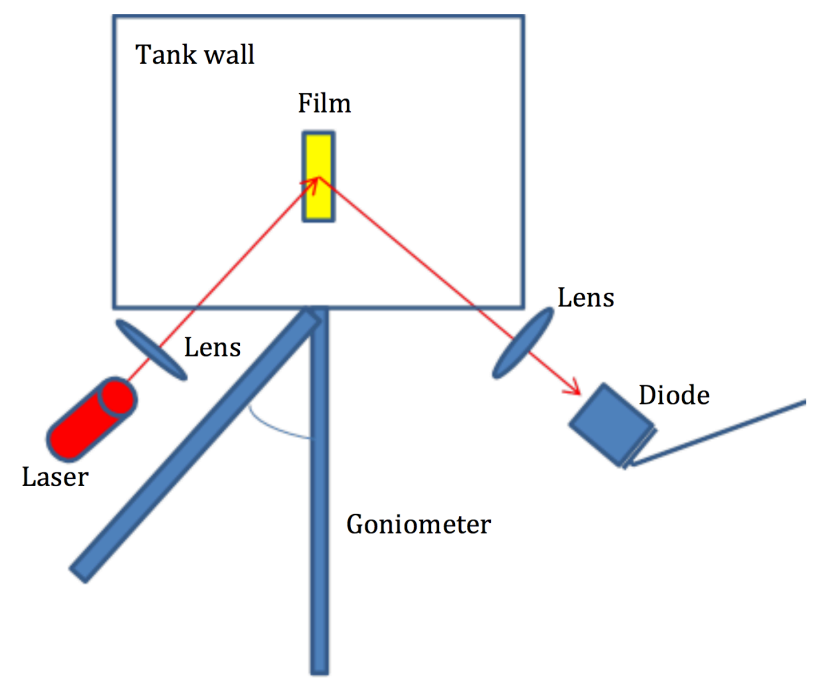

Figure 3.8 Angular incidence system of laser beam using goniometer

Setting the incident angle minimum, we started our measurement. With the $0.5 \mathrm{MHz}$ transducer (transducer number 3 of table 3.2) on, we made the light incident on the film and then it was reflected by the film which was measured (the reflection from the acrylic wall was ignored in our measurement). For the particular incidence angle, we set the diode stand on the optical bench in a way that the reflected ray falls on the diode. We measured the signal strength (amplitude) from the oscilloscope. This way we took our first measurement for the first incident angle. After the first measurement we increased the incidence angle by 0.25 degree and observed the reflected ray amplitude same way as our first measurement. Thus in every measurement we increased the incident angle by 0.25 degree and did so until 82 degree. Since the reflection angle changes every time with the change of incident angle, for each measurement we had to change the diode stand position and had to take the measurement carefully.

For the experiments of this section we used two multi-layer films: a 'Five layer' and a 'Four layer' film. The layers of the 'Five layer film' were: glass-gold-Parylene-gold-SU8. This five layer film was gold coated in the sputter machine for 160 seconds. The layers of the 'Four layer' film were: glass-gold-Parylene-gold. It was gold coated for 180 seconds in the sputter machine. 

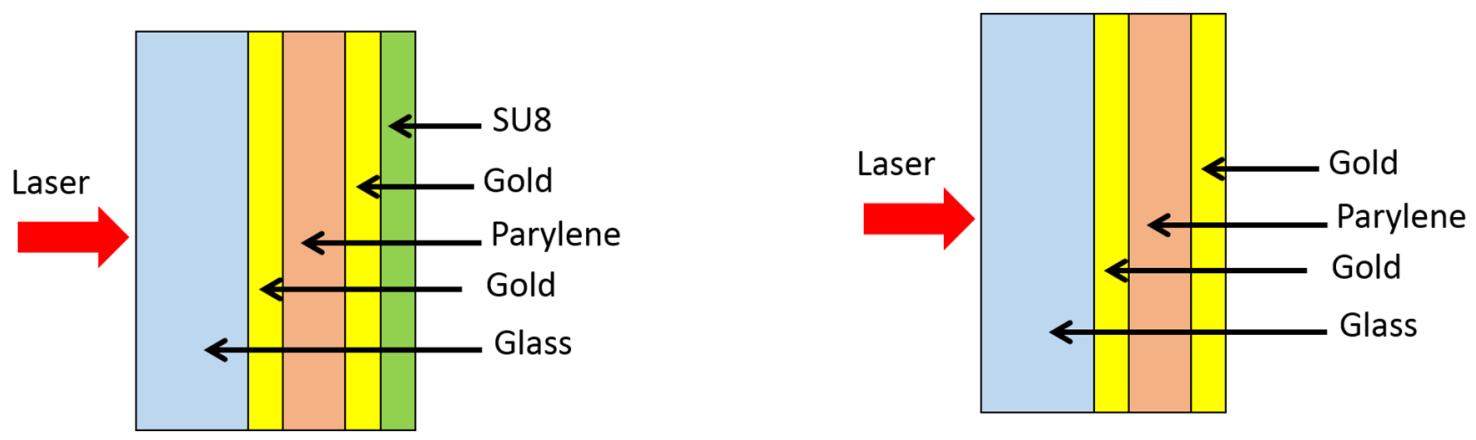

Figure 3.9 (a) Five layer and (b) Four layer Parylene etalons.

\subsubsection{Experiment in a Smaller Light Incident Angle Range using Five Layer Film}

For this experiment, we used the five layer film. We carried on this experiment within the incident angle range (33-53) degree with 0.25-degree intervals. Here we took our measurement for two times. After our first measurement, we repeated the measurement within the same incident angle range, keeping all the conditions same, and recorded all the data.

\subsubsection{Experiment in a Smaller Light Incident Angle Range using Four Layer Film}

For this experiment, we used the four layer film. We carried on this experiment within the incident angle range (33-53) degree with 0.25-degree intervals. Here we took our measurement for two times. After the first measurement, we took out the film from water and then kept it in its previous position. In this new position, we repeated our measurement and recorded all data.

\subsubsection{Experiment in a Larger Light Incident Angle Range using Five Layer Film}

Here we carried on this experiment using the five layer film, in same way as section 3.6.1 but for a larger incident angle range. The range was (8-82) degree, the maximum possible range for our set up. 


\subsection{METHOD OF GOLD THICKNESS MEASUREMENT}

Initially, we made films of different gold thicknesses by varying the 'gold coating time'. Next we took a step to find the correlation between 'gold coating time' (exposure time) and the gold thickness. To do this, we made gold coatings of different exposure times $(20 \mathrm{~s}, 40 \mathrm{~s}, 60 \mathrm{~s}, \ldots 300 \mathrm{~s})$ on some clean glass substrates (same types of substrates that we used to make our etalons).

We assume that for all the samples, the multiplication of each film's resistance and gold coating thickness is a constant [35].

For any sample,

\section{Thickness $*$ Resistance $=$ Constant}

So, If $t_{1}, R_{1} ; t_{2}, R_{2} ; t_{3}, R_{3}, \ldots t_{n}, R_{n}$ be the thickness and resistance of $1^{\text {st }}, 2^{\text {nd }}, 3^{\text {rd }}, \ldots . n$-th sample, then

$$
\mathrm{t}_{1} * \mathrm{R}_{1}=\mathrm{t}_{2} * \mathrm{R}_{2}=\mathrm{t}_{3} \mathrm{R}_{3}=\ldots \ldots=\mathrm{t}_{\mathrm{n}} * \mathrm{R}_{\mathrm{n}}=\text { Constant }
$$

For any two samples,

$$
\mathrm{t}_{1}=\left(\mathrm{t}_{2} * \mathrm{R}_{2}\right) / \mathrm{R}_{1}
$$

Equation 3.2

Knowing the resistance values for all thicknesses and at least one sample's gold thickness (reference thickness), we can find out the gold thicknesses of our all samples.

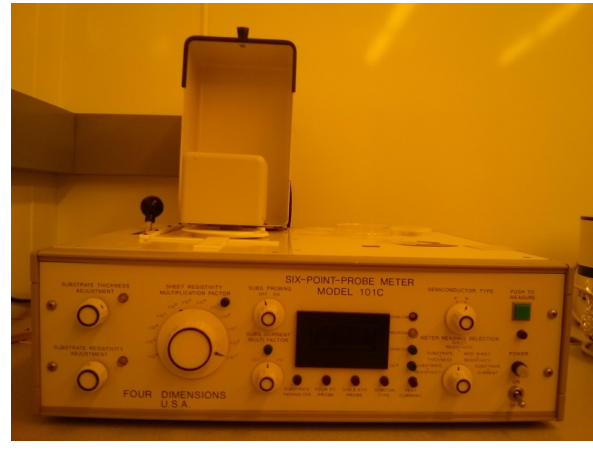

Figure 3.10 Four-probe device

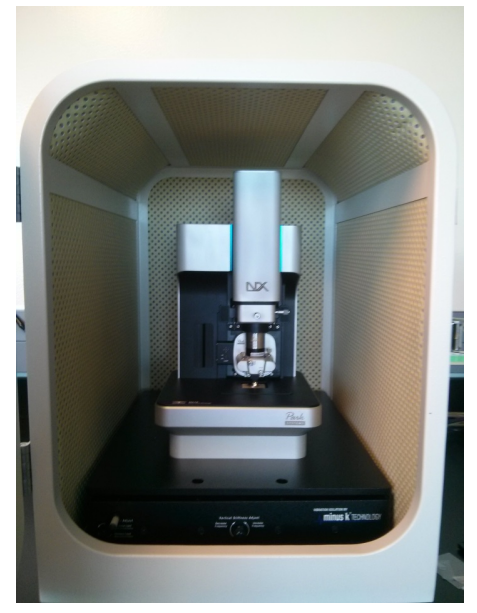

Figure 3.11 AFM device

We measured the gold thickness using two approaches.

(1) Resistance measurement of all films (samples) using the four probe technique. [35]

(2) Measuring the film thickness using an AFM device 
For the first technique, we measured the resistance of all samples (in the unit of $\mathrm{ohm} /$ square) one by one using four-probe device (Figure 3.10) and recorded all the data. For each sample we measured at least 20 different locations of it, recorded all the resistance values and then found the 'mode' number from that values. We considered this mode number as the resistance values of that sample. As we had only one sample of each exposure time, we did not have the chance to repeat this process in another sample with same exposure time. For the second technique, we choose a sample, which was coated for $160 \mathrm{~s}$ in the sputter device, whose thickness was measured by using a AFM device (Figure 3.11). We considered this sample as our reference sample (other sample's thickness was measured using the thickness information of this sample).
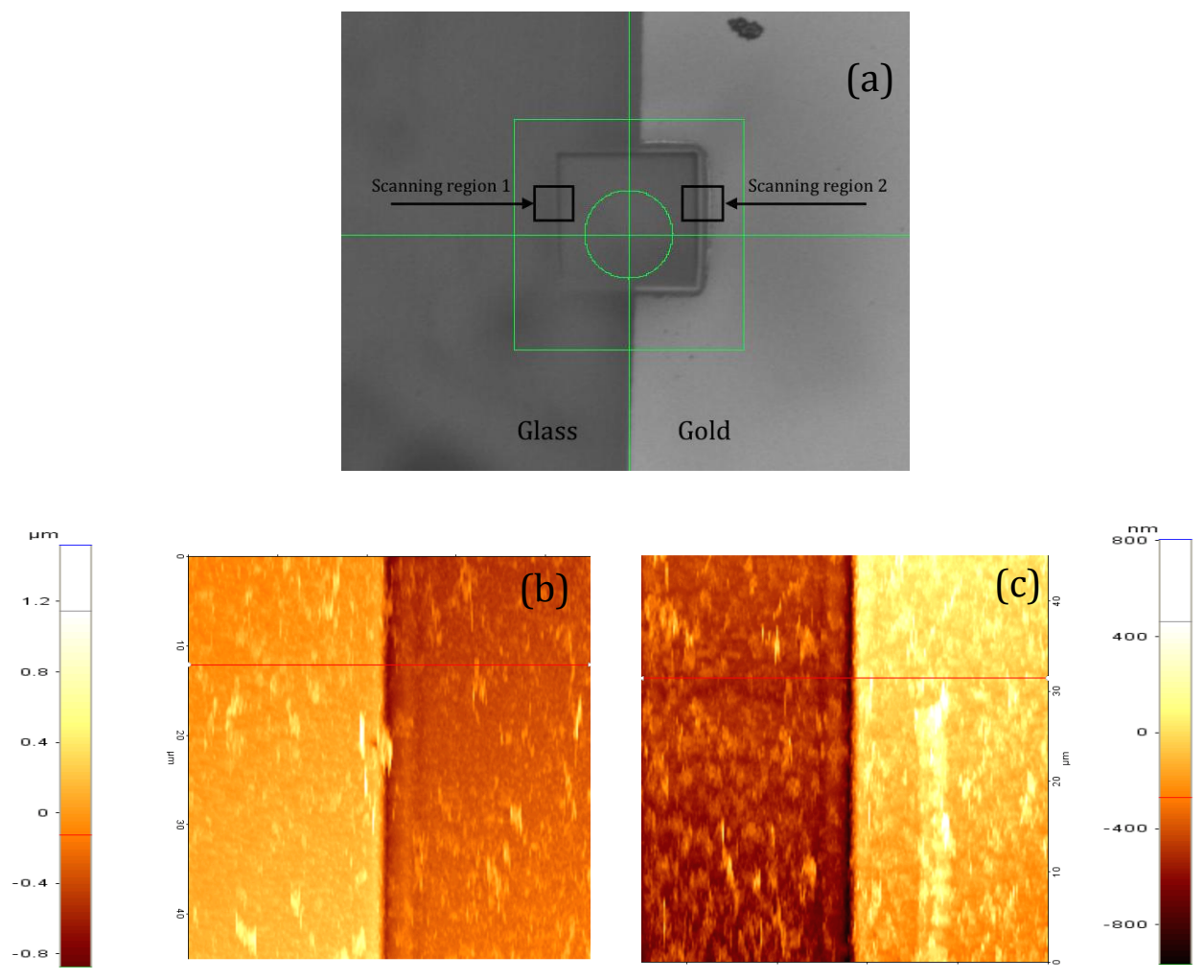

Figure 3.12 (a) A picture taken by the inspection camera showing gold sample where a rectangular hole was created by a rectangular laser pulse on the gold coated-uncoated boundary line. Region 1 and 2 are two selected AFM scan area on two sides of this laser generated hole. (b) AFM scanned picture to find the glass depth (c) AFM scanned picture to find glass with gold layer depth. 
We found a straight boundary line on the sample where one side of this boundary line was with gold coating and another side was without gold (Figure 3.12(a)). We placed the gold coated-uncoated borderline under a rectangular laser pulse (with energy density $\left.2 \mathrm{j} / \mathrm{cm}^{2}\right)$. The laser pulse dimension was $(100 \mu \mathrm{m} * 100 \mu \mathrm{m})$. This pulse made a rectangular hole on it. The hole had same rectangular area as the rectangular pulse. We considered this rectangular hole has a wall in one side who's height is 'without gold layer' and another side has the height 'with gold layer'. We selected two area, 'region 1' and 'region 2 ' in the two sides of the hole for AFM scan. Region 1 scanning result gave us the depth information of glass and region 2 scanning result gave us the depth information of glass and gold together. A line profile (perpendicular to the border line) of each region provides the idea of their step's depth/height graphically. The depth difference of two regions gave us the thickness data of the gold layer on the glass sample. 


\section{Chapter 4 RESULT}

\subsection{PREPARED ETALONS}
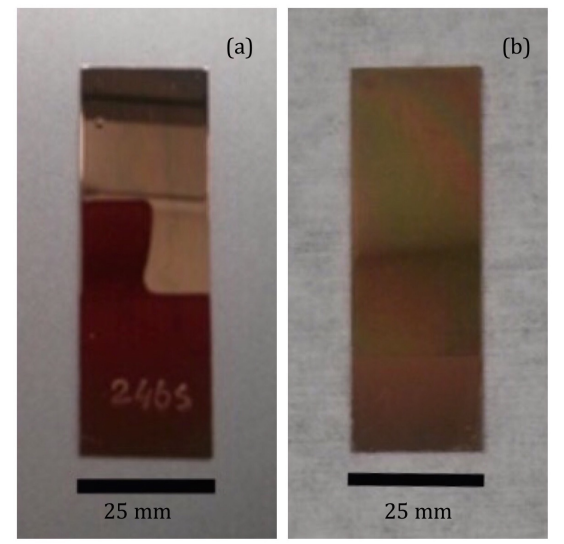

Figure 4.1 Sample of prepared (a) SU8 and (b) Parylene-C etalon

We prepared two types of Fabry-Perot etalons using SU8 and Parylene-C materials . Figure 4.1 show pictures of the two types etalons.

\subsection{OPTICAL CHARACTERIZATION OF ETALONS}

A spectrometer was used to measure the transmission spectrum of the etalons to characterize them. This transmission spectrum is actually a different form and experimental form of the graph of Figure 2.3. The spectrometer in transmission mode gave us the following measurements for the SU8 (Figure 4.2) and Parylene-C (Figure 4.3) films. 


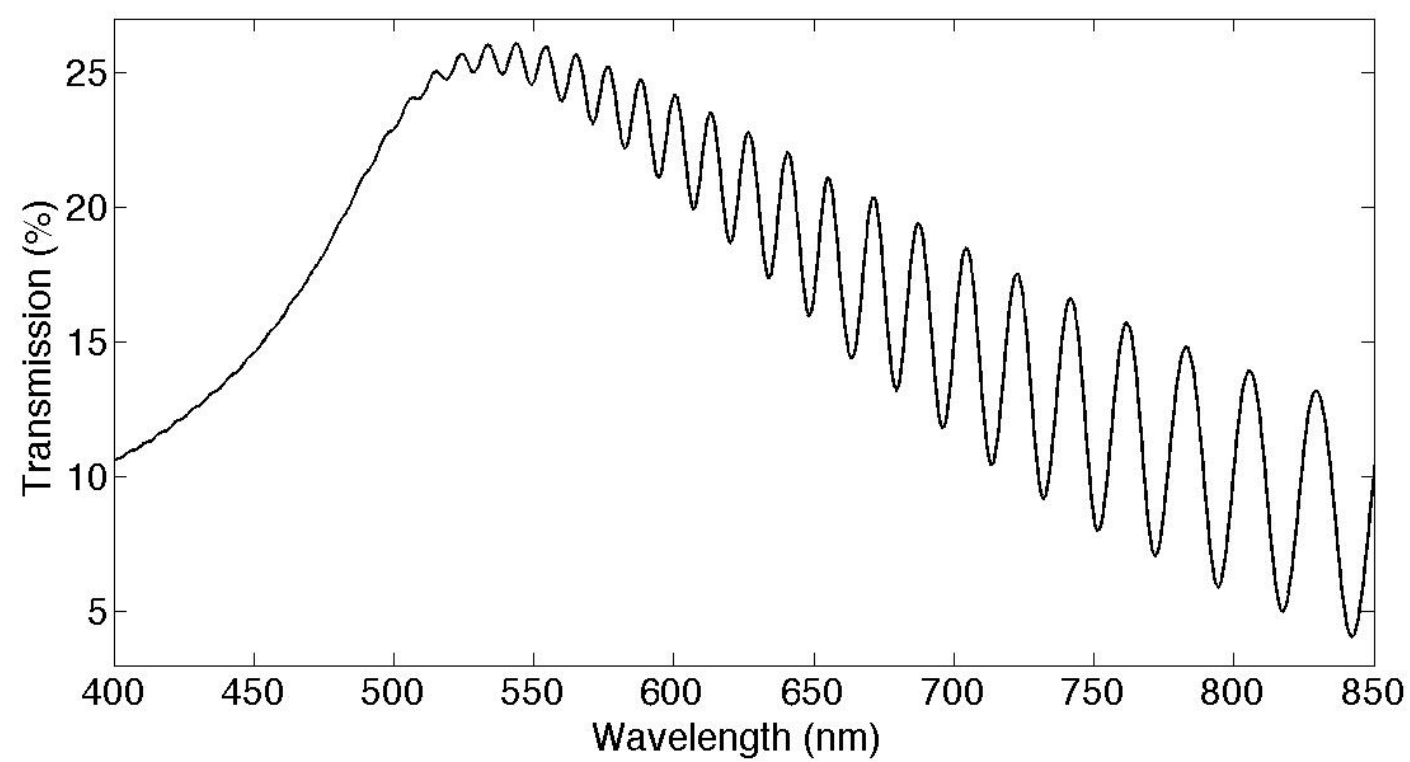

Figure 4.2 Transmission spectrum of the SU8 etalon which was coated with gold for 138 seconds in the sputter device.

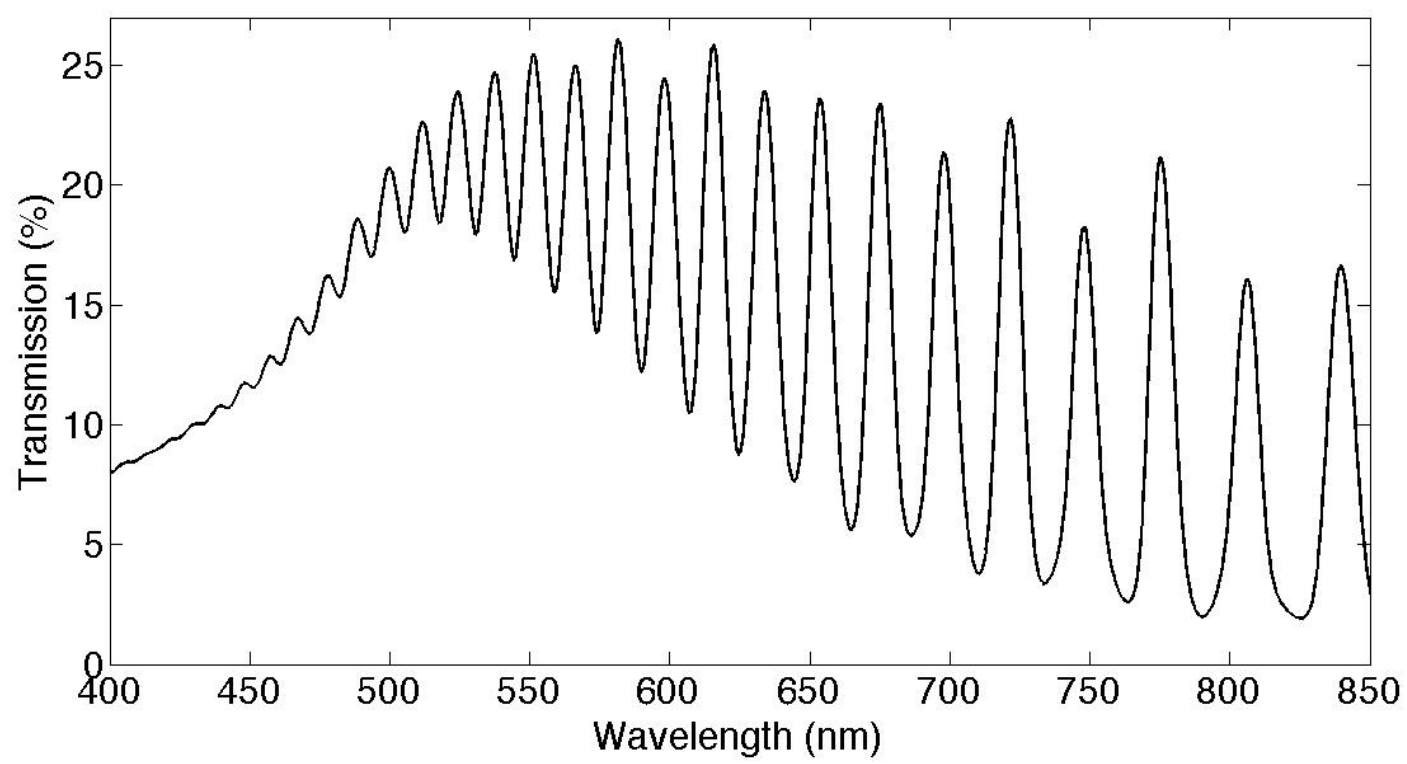

Figure 4.3 Transmission spectrum of the Parylene-C etalon, which was coated with gold for 200 seconds in the sputter device.

In both transmission spectra it was observed that (a) the transmission is maximum at around $500 \mathrm{~nm}$. (b) The graphs have some clear oscillations in the \% transmission. The oscillations start after a certain wavelength. (c) The oscillation amplitudes are larger at longer wavelengths. 
The 'oscillation' in the transmission graphs signifies interference of light in the FabryPerot etalon. A better quality etalon (higher Finesse value, reflectivity close to 1) needs two mirrors with higher reflectivity which results in the strong multiple reflection between the mirrors. If the mirrors do not have significant reflectivity, there will be no significant multiple reflections, and the interference will be weaker. The transmission graphs did not show any 'oscillations' until the visible range. Because the mirror did not have significant reflectivity until this region. This agrees with the experimental graph of gold reflectivity vs. wavelength in [27]. The graph shows that there is no significant reflection until the visible light range $(380 \mathrm{~nm})$. The reflectivity significantly starts to increase from visible light range and it increases continuously until $\sim 1000 \mathrm{~nm}$.

We also observed that the resonance becomes sharper with increasing wavelength i.e. the Finesse increases. Sharper resonance signifies strong reflectivity of the mirrors. The 'Reflectivity vs. wavelength' graph [27] shows that the gold reflectivity increases rapidly from visible (380 $\mathrm{nm}$ to $\sim 1000 \mathrm{~nm}$ ) wavelength range. So it is expected that the mirrors quality will be increasingly better from $380 \mathrm{~nm}$ and should be highest around $1000 \mathrm{~nm}$ wavelength range. Both graphs are showing the same result as our expectation.

In both of our transmission graphs, the adjacent peak-to-peak distance corresponds the thickness of the film. We planned to make a $8 \mu \mathrm{m}$ SU8 and a $6.9 \mu \mathrm{m}$ Parylene film. The thickness estimated from the graphs above are $8.76 \mu \mathrm{m}$ for the SU8 film and $6.61 \mu \mathrm{m}$ for the Parylene film (measured at wavelength $632.8 \mathrm{~nm}$ ), as explained below.

\section{Film thickness calculation}

From the transmission spectra of both type films and using the following formula (formula obtained from Equation 2.1) we estimated the film thickness, $l$, where $\mathrm{n}$ is refractive index.

$$
l=\frac{\lambda_{1} * \lambda_{2}}{\left[2 * n\left(\lambda_{2}-\lambda_{1}\right)\right]}
$$

Here $\lambda_{1}$ and $\lambda_{2}$ were two adjacent chosen peaks near $632.8 \mathrm{~nm}$ and we used the two peaks wavelength information to find the thickness.

Light can transmit through a thin layer of gold with thickness between $4 \mathrm{~nm}-40 \mathrm{~nm}$, but the transmission is not the same for all wavelengths. A characteristics graph [27] of 'Light transmission vs wavelength' shows that a thin gold layer has maximum 
transmission at the visible light range and less transmission in the ultraviolet and infrared region. Our transmission graphs for both types of films confirm this. The etalon can be considered as two thin layers of gold separated by a membrane (we considered the gold layers in our etalon as 'thin').

The transmission spectrum of the film provides information on the etalon quality. The sharper the resonance of the transmission spectrum, the better the quality of the etalon. Another parameter to assess etalons quality is it's 'Finesse' (or quality factor). The Finesse of SU8 etalon and the Parylene etalon were obtained as 2 and 2.07 respectively (measured at $632.8 \mathrm{~nm}$ ). Film quality depends on the film uniformity too.

\section{Finesse $\mathcal{F}$ and Reflectivity $\boldsymbol{R}$ Calculation:}

From the transmission spectra of the film, we measured the Finesse at a particular wavelength. To measure the Finesse at $632.8 \mathrm{~nm}$, we choose two adjacent peaks near $632.8 \mathrm{~nm}$. From these two peaks we calculated the peak-to-peak distance in $\mathrm{nm}$ (this is also called the free spectral range, FSR). As the next step, we choose a peak near 632.8 nm. From that peak, we calculated the FWHM in nm. Equation 2.21 has a form of Finesse, $\mathcal{F}$ which is:

$$
\mathcal{F}=F S R / F W H M
$$

Equation 4.2

And, equation relating reflectivity $(\mathrm{R})$ and Finesse (solution of Equation 2.18) is [47]:

$$
\ln R=-\frac{\pi}{\mathcal{F}}
$$

Equation 4.3

For the SU8 film, the Finesse measured at $632.8 \mathrm{~nm}$ was $2(\mathrm{R}=.21)$ and near $650 \mathrm{~nm}$, it was $1.97(\mathrm{R}=.20)$. By the same process we measured the Finesse of the Parylene film. For the Parylene film, the Finesse measured near $632.8 \mathrm{~nm}$ was $2.07(\mathrm{R}=.22)$, and near 650 $\mathrm{nm}$ it was $2.41(\mathrm{R}=.27)$.

We achieved near same quality for the two types films, but they were made in two different processes. In general, the vacuum deposition technique produces higher quality film than the sputter deposition technique.

The two film materials had very close refractive index. But the SU8 film dissolves in water when it is exposed to water for a long time like 24 hours [21] but the Parylene does not dissolve in water and therefore the Parylene film is not damaged when submerged in water. 


\subsection{OPTICAL DETECTION OF ULTRASOUND}

We used SU8 and Parylene etalons in the experimental conditions of Figure 3.5. The mechanical and optical property of Parylene and SU8 are very close. We can discuss the ultrasound detection mechanism for Parylene case only. Our Parylene etalon thickness, $l$ was $6.61 \mu \mathrm{m}$ (section 2.4.1). The transducer we used initially produced $1 \mathrm{MHz}$ frequency ultrasound. The pressure amplitude value of this ultrasound signal was $P_{0}$ (section 2.4.2) at pulser voltage $200 \mathrm{~V}$. This $P_{0}$ amount of pressure incident on the Parylene etalon, and changed its thickness $d l_{0}$ (Equation 2.33). The $d l_{0}$ thickness change created a phase change of $d \phi$ in the laser output. In consequence this $d \phi$ phase change then made $d I_{0}$ (Equation 2.37) intensity change in the output light beam. With our diode we measured this $d I_{0}$ intensity change with the initial intensity $I_{0}$, in our convenient unit.

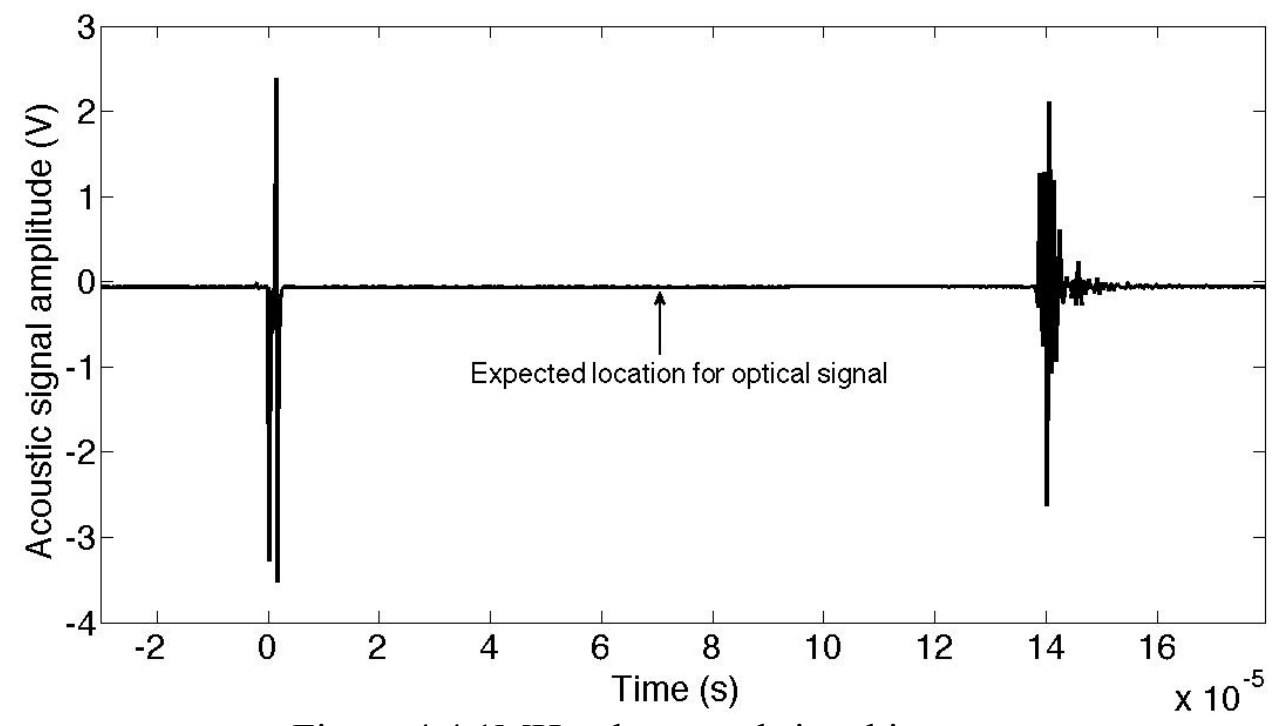

Figure 4.4 1MHz ultrasound signal in water

Using the $1 \mathrm{MHz}$ transducer (transducer number 1 of Table 3.2), we obtained the ultrasound signal of Figure 4.4. Here the initial excitation pulse is recorded at time $t=0$ second. The pulse reflected of the film and then was received by the transducer at $140 \mu \mathrm{s}$. The optical signal is therefore expected to be detected at time $\mathrm{t} \sim 70 \mu \mathrm{s}$.

Using the SU8 film (gold coated for 138s), and a Parylene-C film (gold coated for 200s) in water, we obtained the following two optical signals, which were used to detect the ultrasound signal of Figure 4.4. 


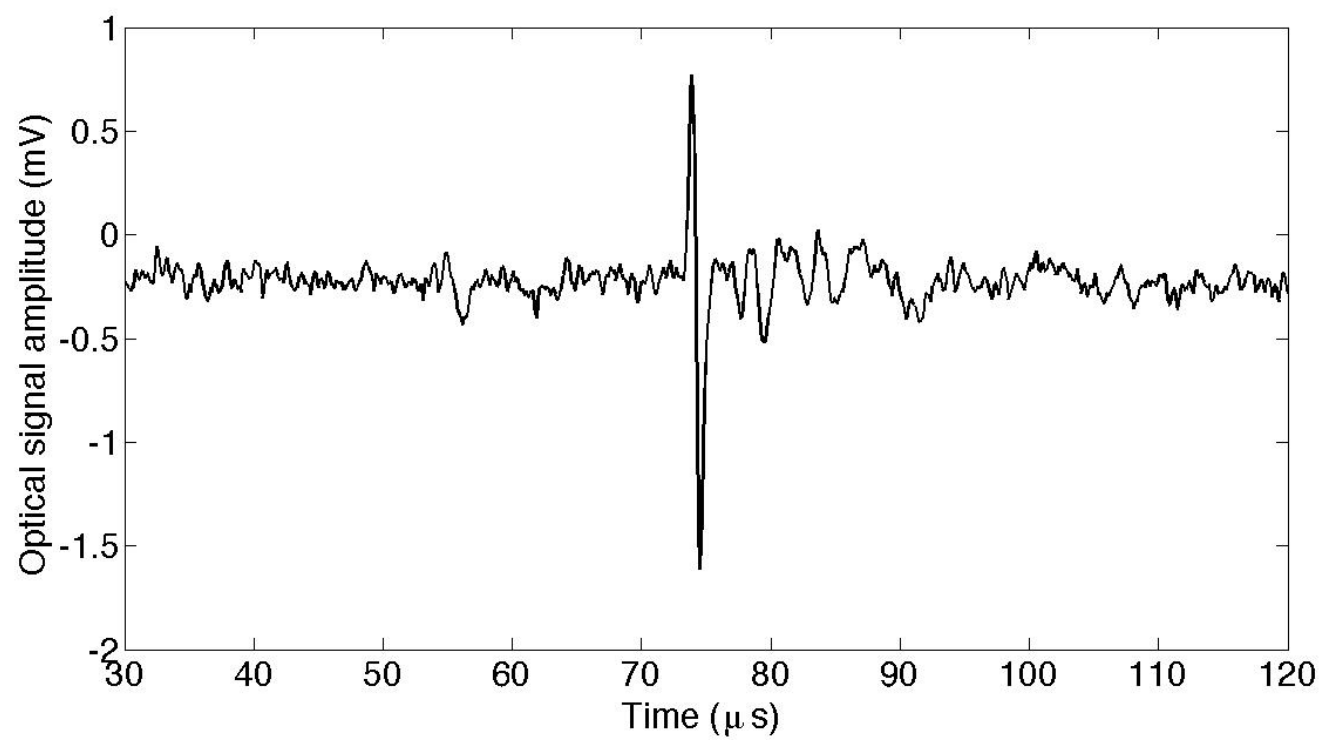

Figure 4.5 Optical signal recorded using the SU8 etalon (gold coated for $138 \mathrm{~s}$ ).

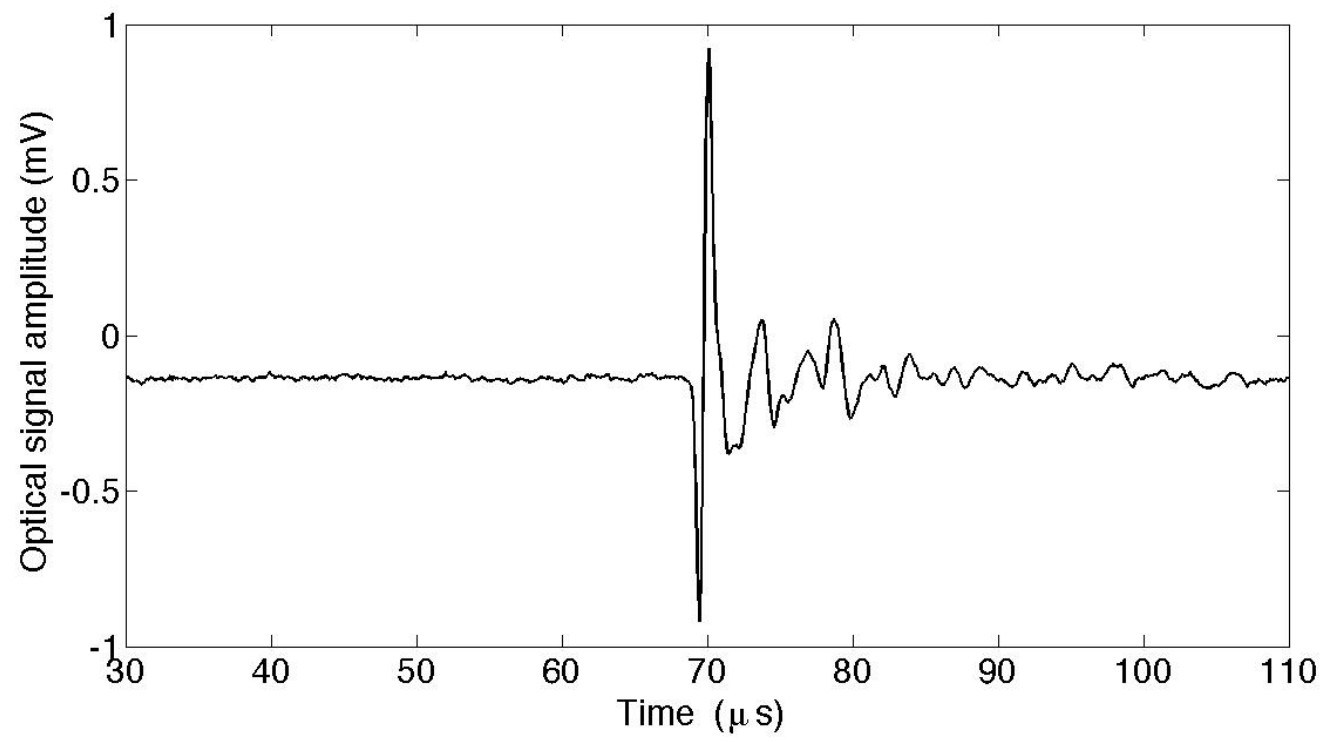

Figure 4.6 Optical signal recorded using the Parylene-C etalon (gold coated for $200 \mathrm{~s}$ ).

The optical signal using SU8 film was detected at a time $74.2 \mu$ s and using the Parylene$\mathrm{C}$ film, it was detected at $69.8 \mu \mathrm{s}$. The signals were detected very close to the expected time, with the signal using the SU8 film slightly delayed. The reason for this delay could be any vibration of our relatively unstable setup. The signal frequencies we obtained were also close to $1 \mathrm{MHz}$ but did not completely match with the ultrasound frequencies. A more rigid set up in a different laboratory was found to remove this mismatch as shown in our next experiments. 
The following table shows the summary of the result of this experiment.

Table 4.1 Summary result of ultrasound detection by etalons

\begin{tabular}{|c|c|c|c|c|c|c|c|}
\hline $\begin{array}{l}\text { Film } \\
\text { Type }\end{array}$ & $\begin{array}{c}\text { Ultra } \\
\text { sound } \\
\text { signal } \\
\text { detection } \\
\text { time }(\mu \mathrm{s})\end{array}$ & $\begin{array}{l}\text { Expected } \\
\text { time } \\
\text { location for } \\
\text { optical } \\
\text { signal }(\mu \mathrm{s})\end{array}$ & $\begin{array}{c}\text { Optical } \\
\text { signal } \\
\text { detection } \\
\text { time }(\mu \mathrm{s})\end{array}$ & $\begin{array}{l}\text { Ultrasound } \\
\text { frequency } \\
(\mathrm{MHz})\end{array}$ & $\begin{array}{c}\text { Optical } \\
\text { signal } \\
\text { frequency } \\
(\mathrm{MHz})\end{array}$ & SNR & $\begin{array}{c}\text { Signal } \\
\text { information } \\
\text { (optical and } \\
\text { ultrasound) }\end{array}$ \\
\hline SU8 & \multirow[t]{2}{*}{140} & \multirow[t]{2}{*}{70} & 74.2 & \multirow[t]{2}{*}{1} & 0.84 & 42.75 & \multirow{2}{*}{$\begin{array}{l}\text { Signals } \\
\text { averaged } \\
\text { over } 4096 \\
\text { times. Pulser } \\
\text { voltage set } \\
\text { to } 200 \mathrm{~V} \text {. US } \\
\text { Gain } 27 \mathrm{~dB}\end{array}$} \\
\hline $\begin{array}{l}\text { Parylene } \\
-\mathrm{C}\end{array}$ & & & 69.8 & & 0.84 & 263.19 & \\
\hline
\end{tabular}

We did this experiment a few more times with different 'transducer-film' distances by moving the transducer. This was done to see whether the optical signal changes with the changing 'transducer-film'. Every time we observed that the optical signal changed with changing 'transducer-film' distances and it always appeared at the middle of this distance. This test further confirms that the optical signal is detecting the ultrasound. Our next experiments on more rigid set up also confirms the ultrasound detection using the etalon, where the optical signals were obtained at the middle of 'excitation-first receiving' ultrasound pulse, and both the ultrasound and optical signals had the same central frequency.

\subsection{OPTICAL DETECTION OF DIFFERENT FREQUENCY ULTRASOUND}

In this section, we are interested in making measurements with transducers that have different frequencies. On the same arrangement shown in Figure 3.5 we carried on this experiment several times by changing the transducer. We varied the frequency of ultrasound signal by changing the transducer and observed the corresponding optical signal due to the ultrasound. We did this experiment using three different transducers. The original frequencies of the transducers were: $1 \mathrm{MHz}, 1 \mathrm{MHz}, 0.5 \mathrm{MHz}$ (Table 3.2). From the two $1 \mathrm{MHz}$ transducers, we used one of them to produced $0.5 \mathrm{MHz}$ frequency ultrasound. 
We made the transducer-film distance equal to the focal length of that transducer and then performed the experiment using the arrangement of Figure 3.5. The film we used here was a Parylene film, gold coated for 200s in the sputter device. Using 3 different transducers we obtained representative optical signals shown in Figure 4.7

Ultrasound signal
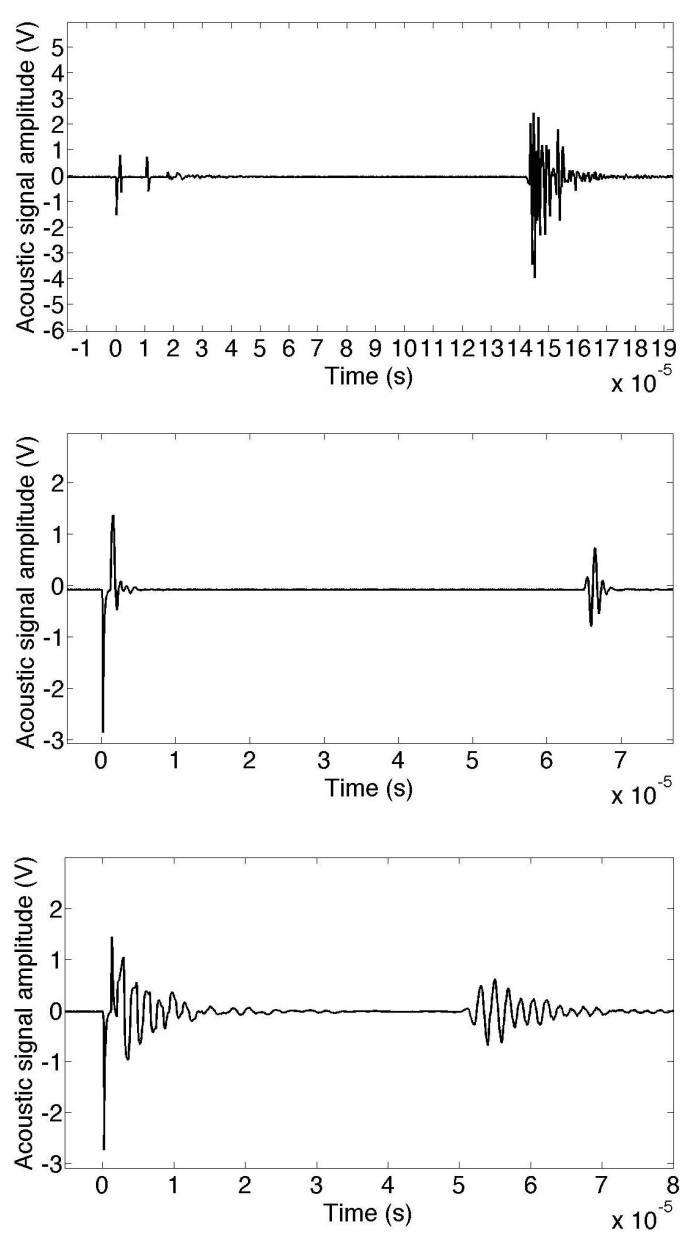

(a)

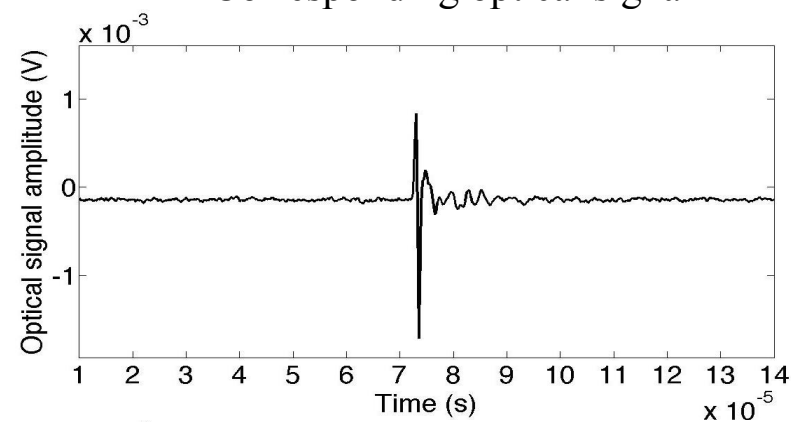

(b)

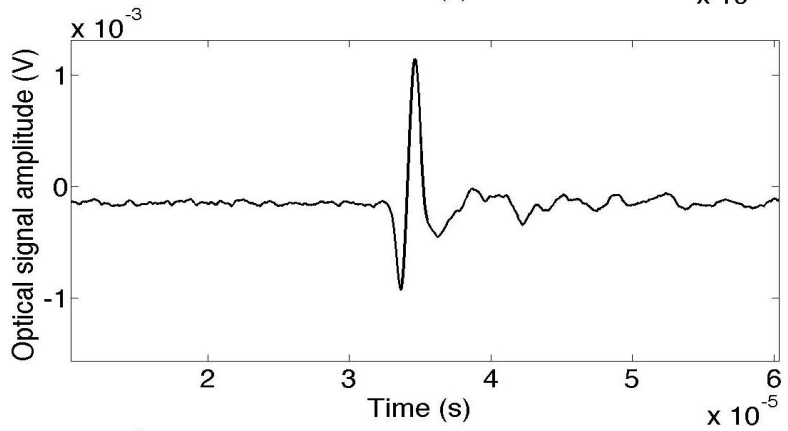

(c)

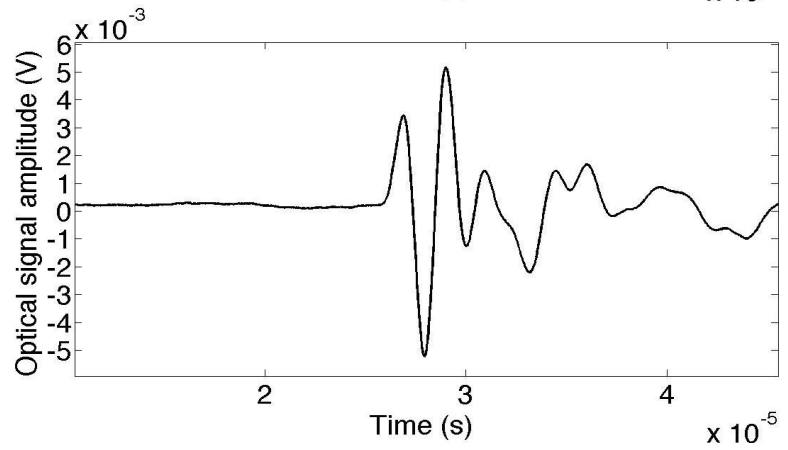

(e)

Figure 4.7 Pulse echo ultrasound signals (a, b, c) of frequency $1 \mathrm{MHz}, 0.5 \mathrm{MHz}$ and $0.5 \mathrm{MHz}$ and their corresponding optical detection signals $(\mathrm{d}, \mathrm{e}, \mathrm{f})$ 
The results of this experiment are shown in Table 4.2

Table 4.2 Result of optical detection of ultrasound using different frequencies

\begin{tabular}{|l|l|l|c|c|c|c|c|}
\hline $\begin{array}{c}\text { Transdu } \\
\text { cer } \\
\text { Serial } \\
\begin{array}{c}\text { Number } \\
\text { (Table } \\
3.2)\end{array}\end{array}$ & $\begin{array}{c}\text { Transducer } \\
\text { produced } \\
\text { frequency } \\
\text { (MHz) }\end{array}$ & $\begin{array}{c}\text { Time location } \\
\text { of first } \\
\text { ultrasound } \\
\text { receiving } \\
\text { pulse (s) }\end{array}$ & $\begin{array}{c}\text { Expected } \\
\text { time } \\
\text { location } \\
\text { for the } \\
\text { optical } \\
\text { signal (s) }\end{array}$ & $\begin{array}{c}\text { Time } \\
\text { location for } \\
\text { the optical } \\
\text { signal } \\
\text { frequency } \\
\text { (s) }\end{array}$ & $\begin{array}{c}\text { Optical } \\
\text { signals } \\
\text { frequency } \\
\text { (MHz) }\end{array}$ & SNR & $\begin{array}{c}\text { Other } \\
\text { Informations }\end{array}$ \\
\hline 1 & 1.0 & $14.53 * 10^{-5}$ & $7.26 * 10^{-5}$ & $7.33^{*} 10^{-5}$ & 1.00 & 186.67 & $\begin{array}{l}\text { Signal } \\
\text { averaged over } \\
4096 \text { times; } \\
\text { Pulser voltage } \\
\text { 200, US Gain } \\
10 \text { dB }\end{array}$ \\
\hline 3 & 0.5 & $6.65 * 10^{-5}$ & $3.32 * 10^{-5}$ & $3.41 * 10^{-5}$ & 0.50 & 136.94 \\
\hline
\end{tabular}

We observed that the optical signal's frequency matches the ultrasound frequency and all the three optical signals have SNR more than 100 (which is considered as a high SNR measurement). This result confirms that our optical system is capable to successfully detect ultrasound frequencies like $1 \mathrm{MHz}$ and $0.5 \mathrm{MHz}$ with a high SNR. 


\subsection{OPTICAL SIGNAL STRENGTH VARIATION WITH INCIDENCE PRESSURE ON ETALON}

In this section we examine whether the optical signal strength changes with incident ultrasound pressure on the etalon. In these experiments, we used the 2 steps measurement (detailed method described in section 3.5). A $1 \mathrm{MHz}$ transducer (transducer number 2 in Table 3.2) and a Parylene etalon (gold coated for $200 \mathrm{~s}$ in the sputter device) were used to this experiment.

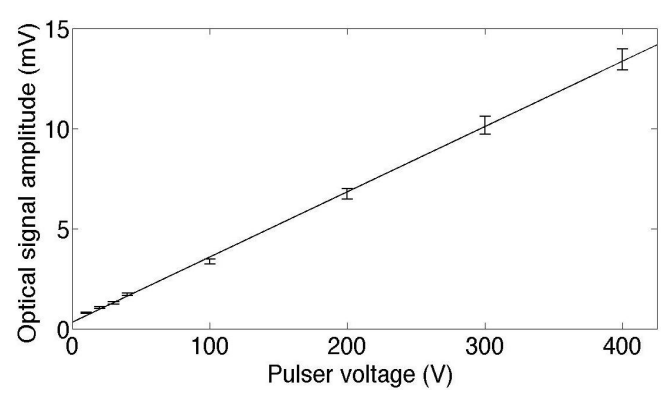

(a)

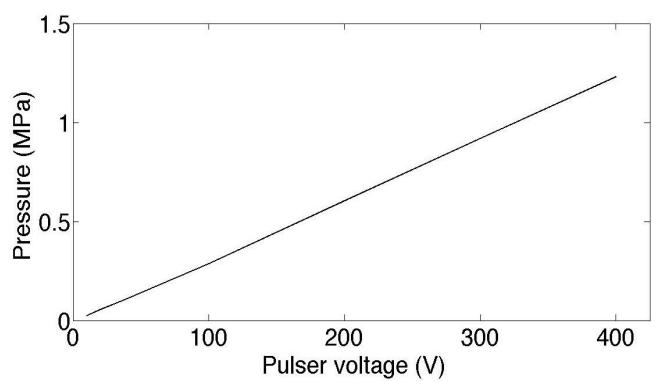

(b)

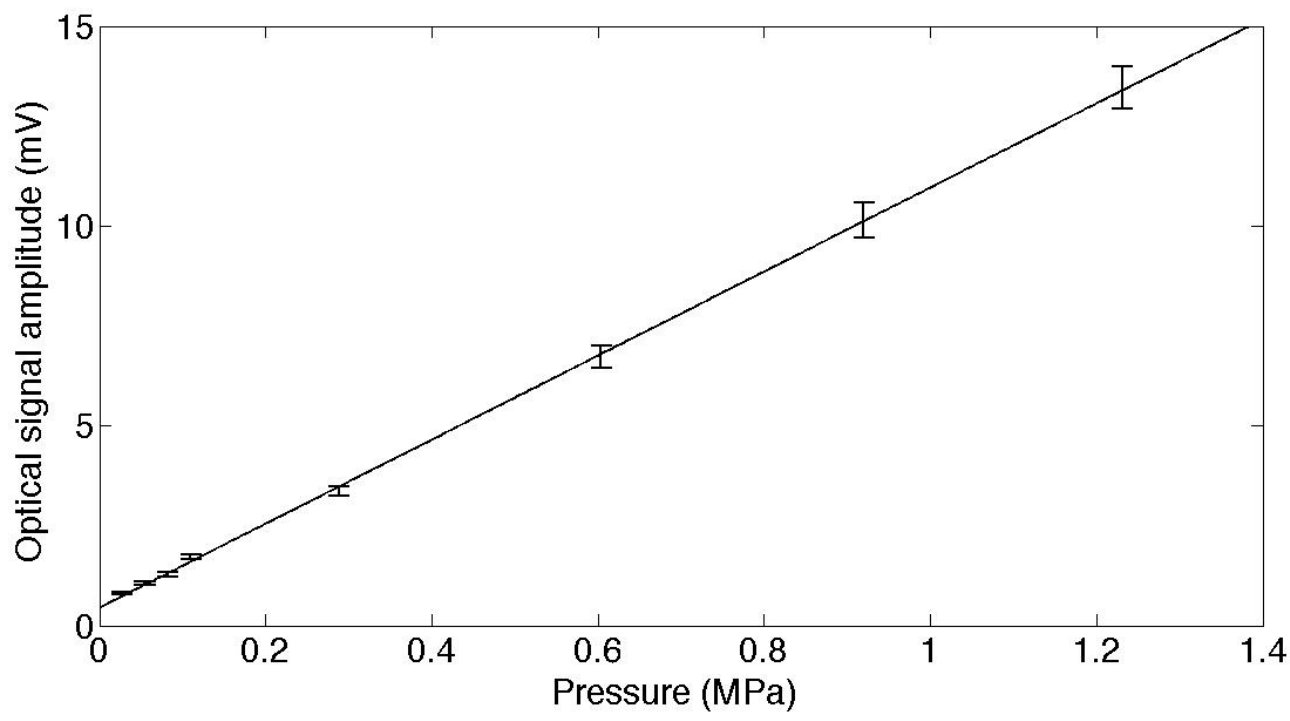

(c)

Figure 4.8 (a) Optical signal amplitude vs pulser voltage (b) Pressure vs pulser voltage (c) Optical signal amplitude vs pressure graph

Following the procedure described in section 3.5 we continued the experiment at a particular pulser votage. We changed the pulser voltage, and observed the optical signal amplitude for each pulser voltage. The pulser voltage was varied from $10 \mathrm{~V}$ to $400 \mathrm{~V}$. We repeated this experiment using the same conditions and set up, every hour for 9 times. We plotted the optical signal amplitude against the pulser voltages. Figure 4.8(a) shows 
this result graphically. The graph shows linearity which suggests that the optical signal strength is proportional to the applied pulser voltage in the range of pressures measured. The higher the pulser voltage, the higher the variability of the optical signal amplitude measurement (as determined by the measurement error bars). Both the ultrasound and the optical signals were averaged over 1024 times.

In the second step measurement, we measured the pressure experienced by the film due to the ultrasound. To do that we used a calibrated hydrophone. We used the setup of Figure 3.5 by replacing the film (which was positioned at the focal point of the transducer) with a hydrophone, so that it experienced the same amount of pressure which was experienced by the film. We changed the pulser voltage and measured the pressure recorded by the calibrated hydrophone. We varied the pulser voltage from $10 \mathrm{~V}$ to 400 volt and observed the pressure each time. We plotted the pressure values against the pulser voltages. The graph shows linearity between these two parameters. The result confirms that pressure on a film is proportional to the applied pulser voltages.

We now combined the result of Figure 4.8(a) and Figure 4.8(b) to see how optical signal amplitude vary with the applied pressure on the film. Using the X-axis data of these two figures, we plotted optical signal amplitude vs pressure (Figure 4.8(c)). Here again we see a linearity between these two parameters. The result establishes that the optical signal strength is proportional to the pressure exerted on a film.

\subsection{REFLECTED LIGHT INTENSITY VS OBLIQUE LIGHT INCIDENCE : THEORETICAL MODEL}

An etalon performance depends on the light wavelength. Usually a tunable continuous laser is needed to set the etalon at the best performance point. However, this type of laser was not available in our lab. So the dependence of the etalon performance on the light wavelength was demonstrated indirectly by measuring the signal at various light incidence angle. This was possible because an interferometer output is a function of $\lambda$ and angle in the form of phase difference $\delta$ only (Equation 2.1). In this section we described 
the theoretical results of reflected light intensity with the variation of light incidence angle, using two multilayer films modeling the films that were used in the experimental condition of section 3.6. For modeling we used the code "TransferMatrix" developed by Professor Andreas B, Dahlin's group at Chalmers University of Technology [38]. The code uses the transfer matrix method to calculate Fresnel coefficients, i.e. transmission, reflection and absorption in an arbitrary thin film multilayer system. We have tested the validity of this code by calculating the transmission and reflection characteristics of a number of different multilayer structures. The code was able to provide expected results in each case.

We considered a 4 layer structure (glass-gold-Parylene-gold) and a 5 layer structure (glass-gold-Parylene-gold-SU8) in this work. The Parylene thickness was set to $6.9 \mu \mathrm{m}$ and SU8 layer was set to $8 \mu \mathrm{m}$. We choose this particular Parylene thickness because the fabrication process we used have a reliable recipe for deposition of $6.9 \mu \mathrm{m}$ thick Parylene film. However, we were free to choose the gold thickness, the angle of light incidence and polarization of light. Therefore in this analysis we focused on the effects of these three parameters on the Fabry Perot performance. Light incident on the etalon can have arbitrary polarization, but can always be decomposed into $\mathrm{p}$ and s polarization. According to our convention the electric field is aligned along and normal to the plane of incidence for the $\mathrm{p}$ and $\mathrm{s}$ polarization, respectively. The expression for Fresnel coefficient for the two polarization is different [51]. In addition two phenomena: complete transmission at Brewster angle and excitation of surface plasmon resonance [53] happens only for $p$ polarized light. Therefore, it is important in both analysis and experiment to know or control the polarization of incident light. In our analysis we varied the gold thickness from $10 \mathrm{~nm}$ to $110 \mathrm{~nm}$ and considered both $\mathrm{p}$ and s polarization. 
Four Layer Etalon
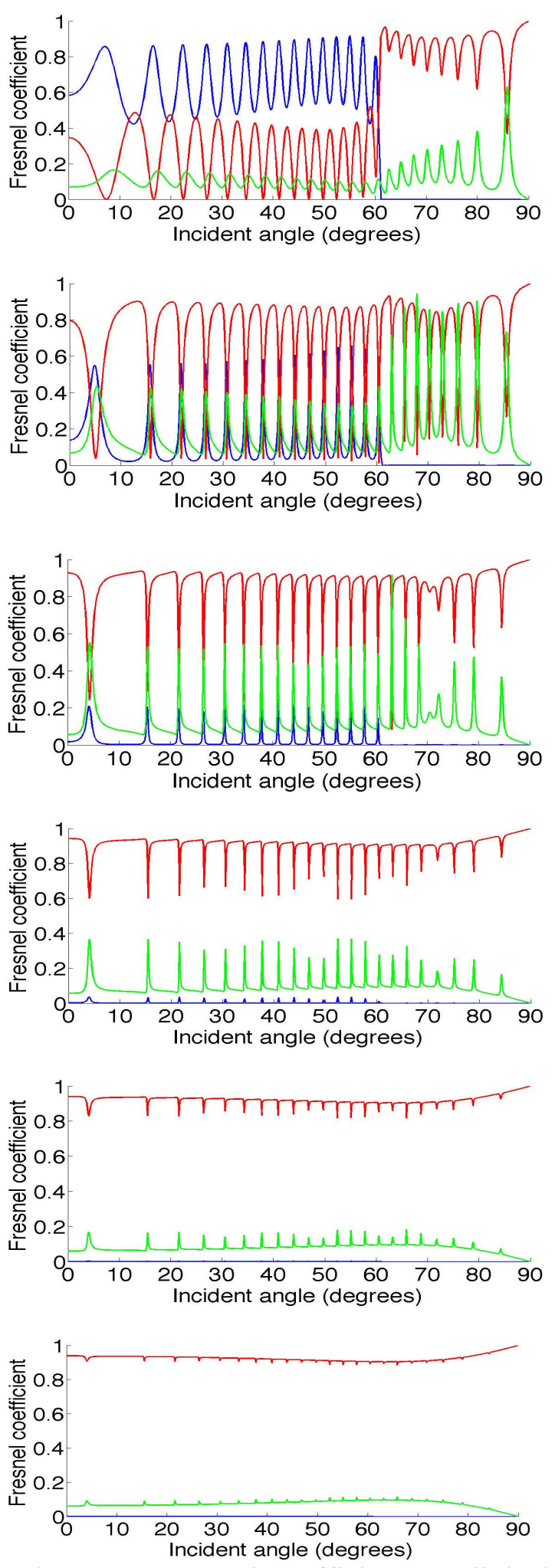

Five Layer Etalon

(a)

$10 \mathrm{~nm}$

Gold

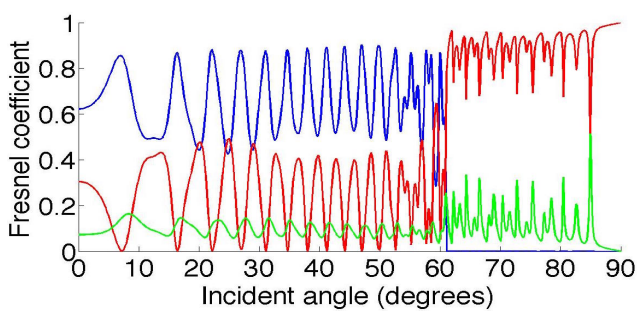

(g)

$10 \mathrm{~nm}$

Gold

(h)

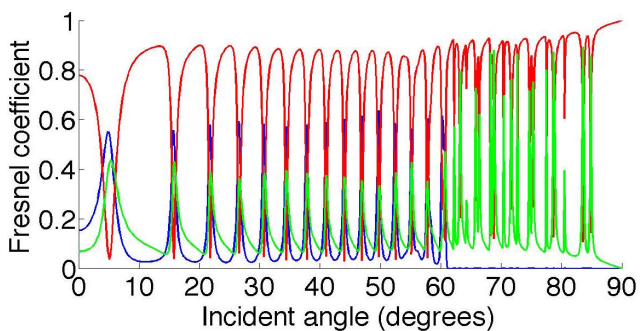

30nm

Gold

(i)

$50 \mathrm{~nm}$

Gold

(j)

$70 \mathrm{~nm}$

Gold

Gold

(e)

90nm

Gold

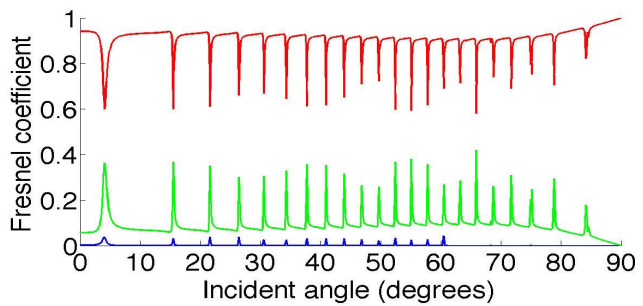

(k)

90nm

Gold

0.4

这 0.2

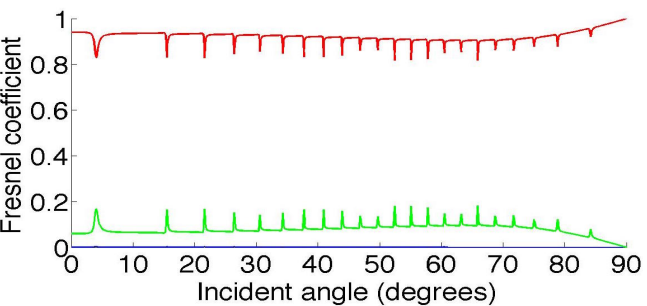

(1)

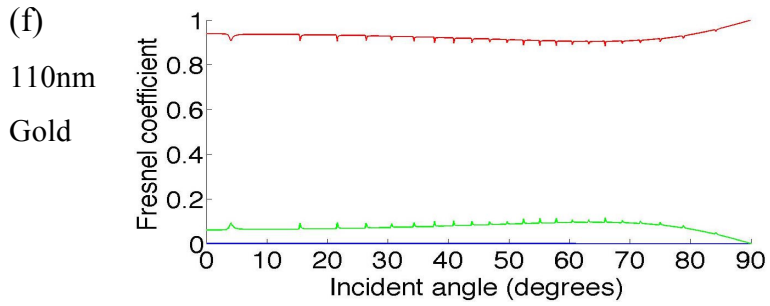

$110 \mathrm{~nm}$

Gold

Figure 4.9 Fresnel coefficients vs light incident angle for p polarized light. The coefficient of reflection, transmission and absorption are presented by red, blue and green curves. 
Four Layer Etalon
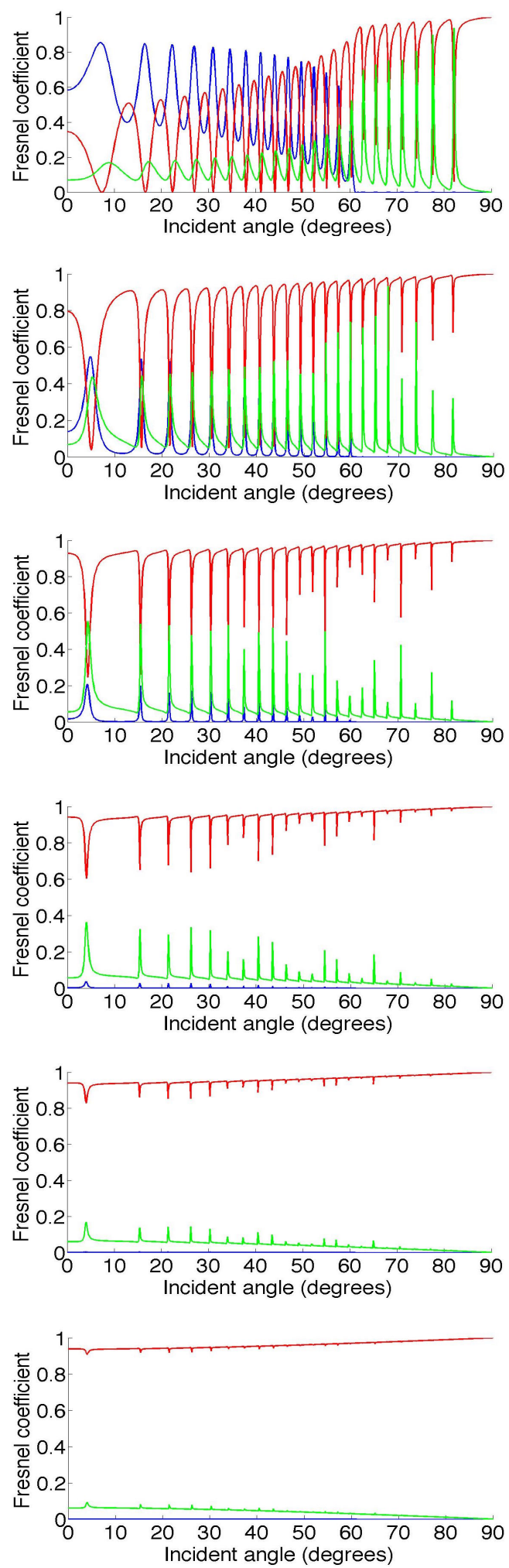

Five Layer Etalon

(a)

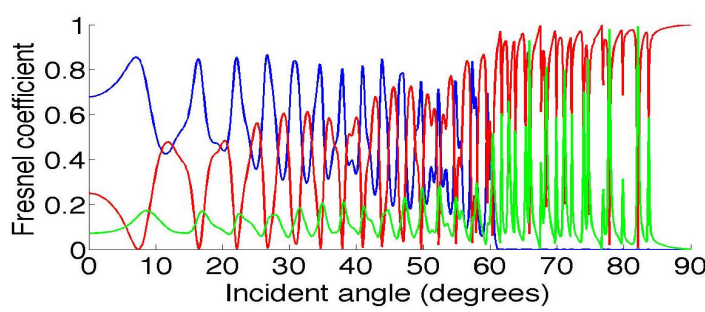

(g)

$10 \mathrm{~nm}$

Gold

(b)

$30 \mathrm{~nm}$

Gold

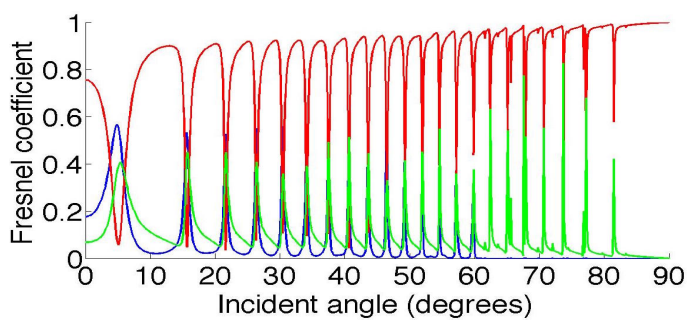

(h)

$30 \mathrm{~nm}$

Gold

(c)

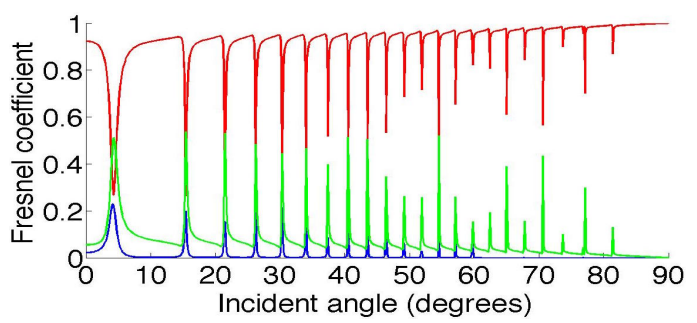

(i)

$50 \mathrm{~nm}$

Gold

Gold

(d)

$70 \mathrm{~nm}$

Gold

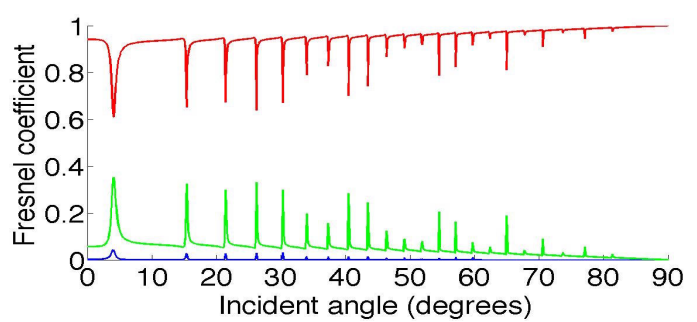

(j)

$70 \mathrm{~nm}$

Gold

(e)

$90 \mathrm{~nm}$

Gold

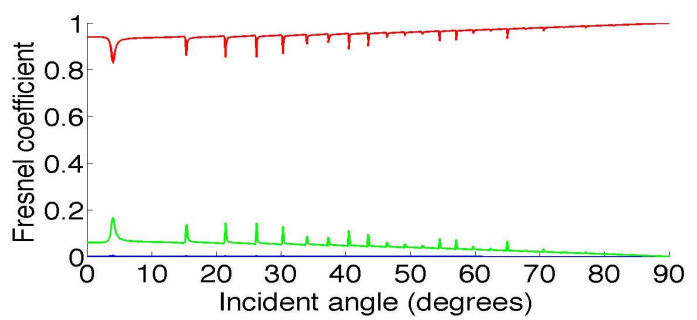

(k)

$90 \mathrm{~nm}$

Gold

(f)

110

$\mathrm{nm}$

Gold

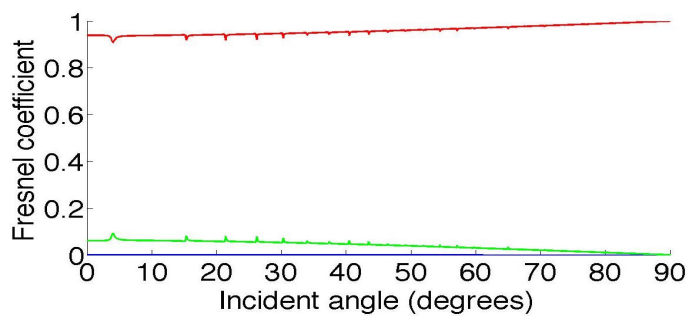

110

$\mathrm{nm}$

Gold

Figure 4.10 Fresnel coefficients vs light incident angle for s polarized light. The coefficient of reflection, transmission and absorption are presented by red, blue and green curves. 
Figure 4.9 shows the results for a number of gold thicknesses for $\mathrm{p}$ polarizations. For a very small thickness, a significant amount of light transmits the gold film (for example 10 nm thick gold, Figure 4.9 (a) and (g)). The gold film in this case does not act as an effective mirror, and the resonances are not sharp. As the gold thickness increases, the resonances becomes sharper (Figure 4.9 (b) (c), (d); (h), (i) and (j)). However if the gold thickness is too large, for example $110 \mathrm{~nm}$ (Figure 4.9 (f) and (1)), almost no light can enter the cavity. Therefore, although the reflectivity is very large, the Fabry Perot effect is almost completely lost in this case and the structure acts like a simple metallic mirror. One interesting feature for all these plots is a large drop of transmission around an angle of incidence of $61^{\circ}$. This is not unexpected. As light travels through different interfaces, its angle of incidence at successive interface changes. According to our calculation for a angle of incidence of $61^{\circ}$, light hits the last interface at an angle greater than the critical angle. As a result transmission out of the cavity becomes very small.

Figure 4.10 shows the reflection, transmission and absorption spectra of the above mentioned structured for the s polarization. The general features for these plots are similar to those of Figure 4.9, although the numerical values are different. Similar to the previous cases, the resonances are sharp for an intermediate layer of gold film thickness.

The result of Figure 4.9 and Figure 4.10 show us that Fabry-Perot which has $30 \mathrm{~nm}$ gold thickness can perform as the best quality Fabry-Perot interferometer among all these gold thicknesses. 


\subsection{REFLECTED LIGHT INTENSITY VS OBLIQUE LIGHT INCIDENCE: EXPERIMENTAL RESULT}

Using the experimental set up of Figure 3.8 we measured the dependence of the ultrasound modulated optical signal on the incidence angle. These results can indirectly show the dependence of the ultrasound modulated optical signal on the incidence light wavelength. On this set up we did 3 experiments and for all these three cases, we used a $0.5 \mathrm{MHz}$ transducer (transducer serial number 3 of Table 3.2). In our first part, we used a five layer film, gold coated for 160s, experimented with a 33-53 degree light incidence angle range; in the second part, we used a 4 layer film, gold coated for 180s where we varied the light incidence angle by (33-53) degrees. In third part we used the previous 5 layer film with 160s gold coating, with a wider light incident angle range (8-82) degrees. The results are showed in the following three sections.

\subsubsection{Experiment in a Smaller Light Incident Angle Range using Five Layer Film}

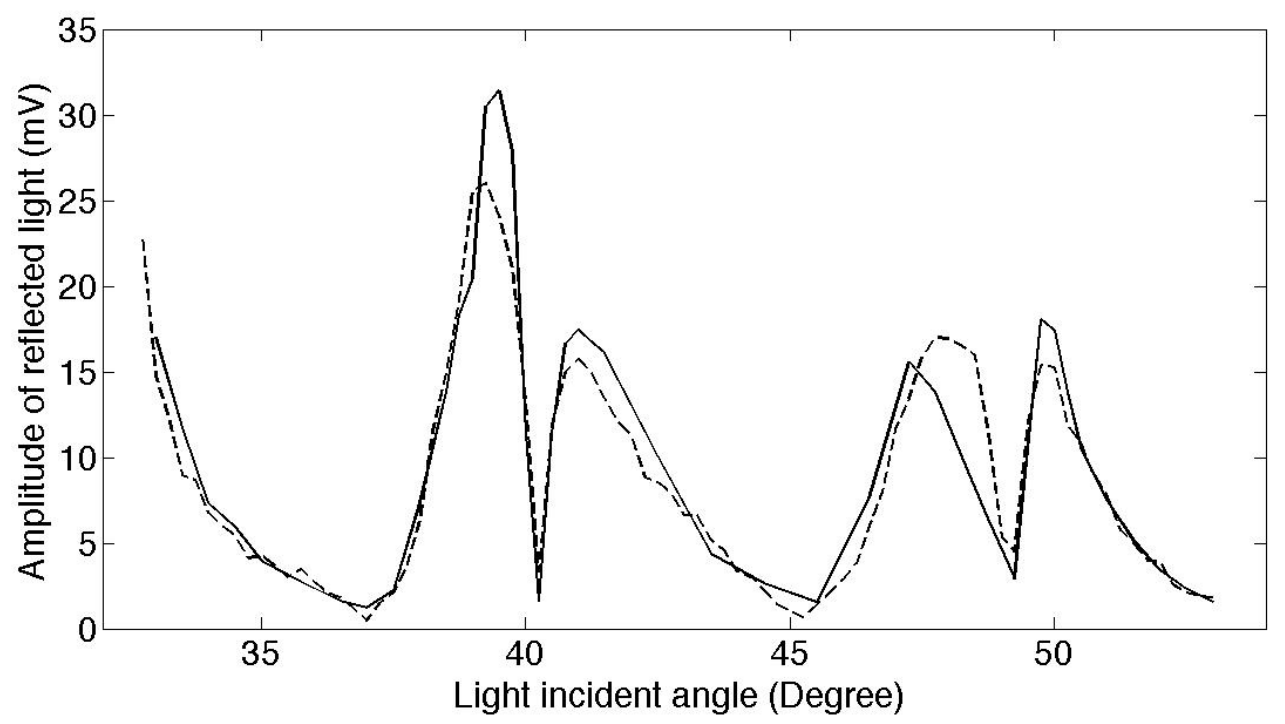

Figure 4.11 Reflected light amplitude vs. light incident angle result using five layer Parylene film in the light incident angle range (33-53) degree. The solid and dotted lines are representing two times measurements of same experiment on same condition.

The graphical result of Figure 4.11 shows peaks of reflected light as a function of incidence angle. The experiment was carried in duplicate with the same conditions gave us almost same curve with small variations. Over all, the maxima and minima position are found same in the two trials. 


\subsubsection{Experiment in a Smaller Light Incident Angle Range using Four Layer Film}

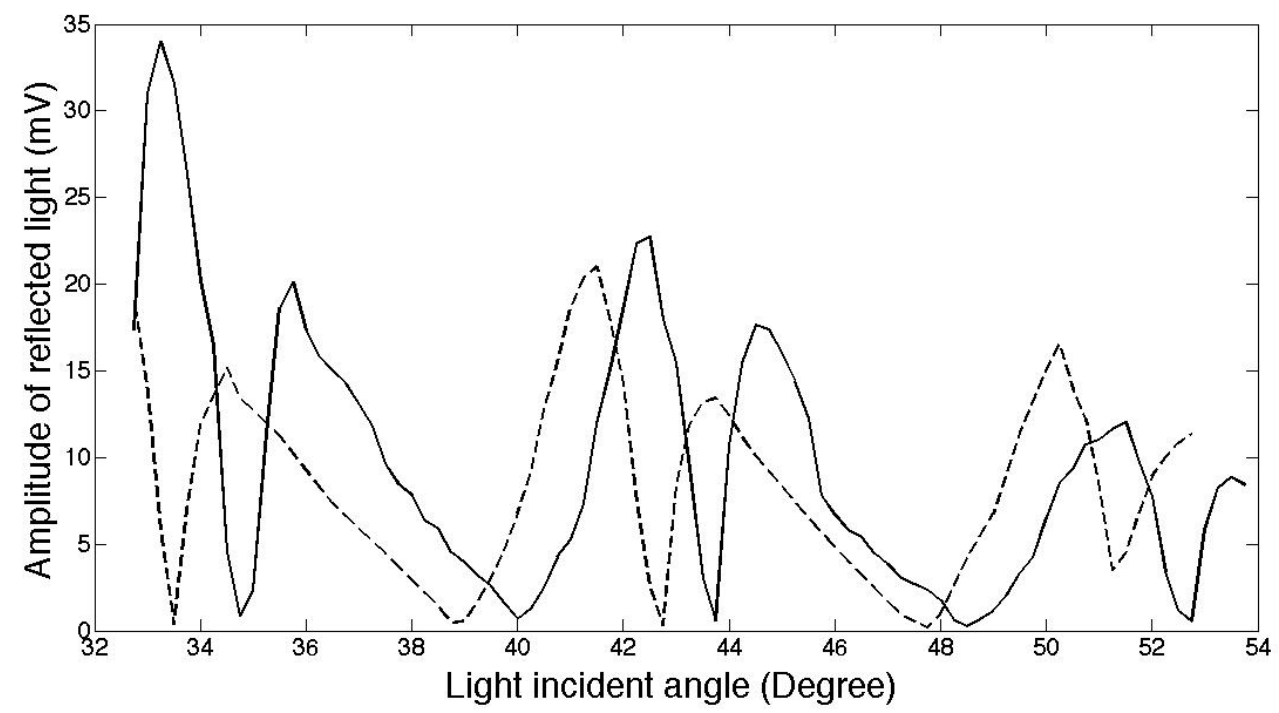

Figure 4.12 Reflected light amplitude vs light incident angle result using four layer Parylene film in the light incidence angle range (33-53) degree. The solid and dotted lines represent two times measurements of same experiment under same condition where the Parylene films position was slightly varied in second time.

The graphical result of Figure 4.12 shows two measurements of the experiment where in second time measurement we just took out the film from water and then replaced it again in its previous position. In our first trial we obtained a curve with some peaks with decreasing amplitude to the higher incident angle. In our second trail, we got almost the same curve but it shows that the curve is shifted to the right. 


\subsubsection{Experiment in a Larger Light Incident Angle Range using Five Layer Film}

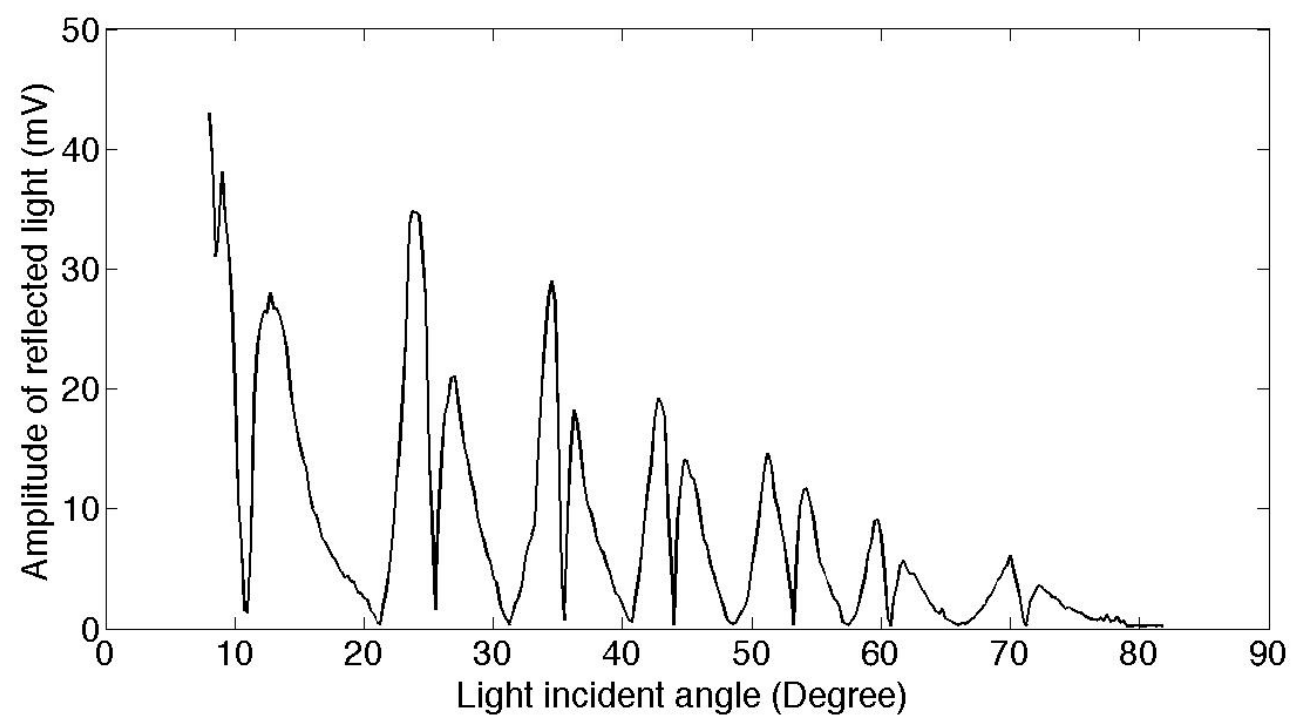

Figure 4.13 Reflected light amplitude vs light incident angle using a five layer Parylene film interferometer in the light incident angle range of 8-82 degrees.

We repeated the experiment of section 4.7.1 using same five layer interferometer. This time instead of (33-53) degree incident angle range we used the maximum incidence angle range which was possible for our set up. We used 8 to 82 degree incident angle range for this experiment and obtained the results presented in Figure 4.13. Here also the peak set has gradually decreasing amplitude to the higher incidence angle.

The experimental result of Figures 4.11, Figure 4.12, and Figure 4.13 are comparable with their simulation results of Figure 4.9 and Figure 4.10 [52]. In our experimental results a series of maxima and minima were observed, which is that same as their corresponding simulation results. For all the three cases, the major mismatch we observed in our experimental results with the model are peaks amplitude. The peaks of the experimental results are gradually decreasing to the higher incidence angle where in the modeling the peaks height (generally) increased to the higher incidence angle.

Differences in the theoretical and experimental results in our experiment can be due to several factors. The simulations are only related to the optical sensitivity of the etalon. The amplitude of the measured signals depends on both the optical sensitivity and 
acoustic sensitivity. We have not studied the dependence of the acoustic sensitivity on the light incidence angle. This can be a future study topic. In our simulation results light passed through a 5 layer media where the glass substrate was the first media. In the experiment, there were other media before the substrate, the element of acrylic tank wall which we did not consider. The etalon was placed attached to the wall of the acrylic tank, which had reflection property and its reflection property was a function of light incidence angle. The acrylic reflection can be ignored for lower light incidence angle, but for higher incidence angle, it cannot be ignored [39].

In our experiment, we attached the etalon substrate to the acrylic wall with a single clip from the top of the interferometer. It made a very thin wedge-like gap between the interferometers substrate and the tank wall. During the experiment, water may have entered this gap and created another wedge shape water layer along with the multilayer system. Water is a material whose reflectivity changes with the incidence angle also [40] and we did not consider this in our simulations.

In our experiment, we did not use any polarizer, and as a result the light was most likely unpolarized. However, the modeling was done for fixed polarization states (either p or s). More experiments with the inclusion of a polarizer in the optical measurement set up would provide a better match between theory and experiment. 


\subsection{REFLECTED LIGHT INTENSITY VS WAVELENGTH: THEORETICAL TRANSFORMATION}

We here wanted to obtain the corresponding 'Reflected light intensity vs wavelength' graph using the experimental result of Figure 4.13, through a theoretical transformation. For Figure 4.13 we recall Equation 2.1:

$$
\delta=\left(\frac{2 \pi}{\lambda}\right) 2 n l \cos \theta
$$

Equation 4.4

Here $\lambda$ was the wavelength of light we used in our experiment $(650 \mathrm{~nm})$, and $\theta$ was the final transmission angle in Parylene film for any corresponding initial angle $\theta_{0}$ (in air). Light practically traced the media as (gold layer ignored):

Air $>$ acrylic $>$ glass $>$ Parylene $>$ water

Light incident from air and transmitted into acrylic media. Here light ray in acrylic was the incidence ray for acrylic-glass interface. Similarly light ray in glass was the incidence ray for glass-Parylene interface. The angle with which light ray finally reached to Parylene layer was $\theta$. Applying Snell's law successively we got the final transmission angle $\theta$ (in Parylene) from an initial incidence angle $\theta_{0}$ (in air).

Now we were willing to obtain 'Reflected light intensity vs. wavelength' for a normal light incidence case. So, Equation 4.4 got the form:

$$
\delta=\left(\frac{2 \pi}{\lambda_{\text {new }}}\right) 2 n l
$$

Equation 4.5

Here $\lambda_{\text {new }}$ were new wavelengths we were going to obtain after the transformation.

As we were going to obtain 'Reflected light intensity vs. wavelength' from the graph 'Reflected light intensity vs. light incidence angle' (Figure 4.13), the phase difference would be same in both cases. So equating Equation 4.4 and Equation 4.5, we obtained:

$$
\lambda_{\text {new }}=\frac{\lambda}{\cos \theta}
$$

Equation 4.6

Now for each incidence angle of Figure 4.13 we obtained a corresponding wavelength $\left(\lambda_{\text {new }}\right)$ value. Using the same Y-axis values (reflected light intensity values) of Figure 4.13 and the new wavelength values of Equation 4.6, we obtained the following graph (Figure 4.14). 


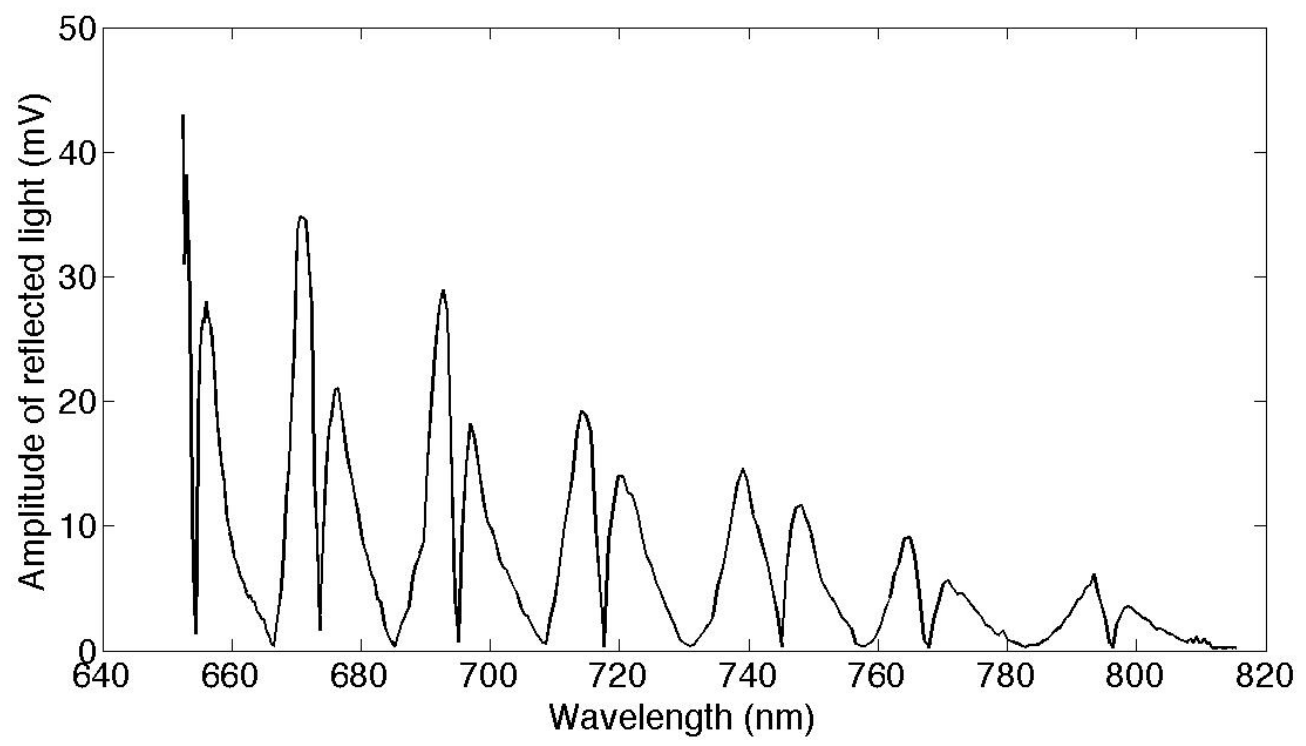

Figure 4.14 Reflected light intensity vs wavelength plot obtained from Fig 4.13 through a theoretical transformation.

Comparing Figure 4.13 and Figure 4.14, we observed, both graphs have same shape, and a set of peaks with decreasing amplitude from left to the right. The decreasing trend in the light intensity vs wavelength may not be correct. The main point of this experiment was to demonstrate indirectly that the amplitude of the ultrasound modulated signals strongly depends on the light wavelength. An optimized wavelength can enhance the signals significantly.

There were many reasons that the decreasing trend in the light intensity vs wavelength might not be correct. The FP theory assumed the film was uniform and the light beam was uniform and infinitely wide. In reality, the film surface might not be smooth and uniform. The light beam had a finite width and Gaussian shape. These would made the experiment results in the oblique incidence deviating from FP theory. 


\subsection{REFLECTED LIGHT INTENSITY VS WAVELENGTH (NORMAL LIGHT INCIDENCE): THEORETICAL MODEL}

In this section we presented the variation of reflectivity as a function of wavelength for normal light incidence for the four and five layer structures (Figure 4.15). We considered only p polarization here.

The general trend of these plots is similar for both the four and five layer structures. For a $10 \mathrm{~nm}$ thick film, the metal does not act as a good mirror and the Fabry-Perot resonance is not very strong. This is evident from the low peak reflectance over the entire wavelength range. For thick gold film, for example $110 \mathrm{~nm}$ film thickness, the reflection becomes larger, but very little light can enter the cavity. As a result the maxima and minima in the spectrum becomes almost indistinguishable. On the other hand for intermediate film thickness ( 30 or $50 \mathrm{~nm}$ ), the peak reflection is high and also the contrast between maxima and minima is also large. So these thicknesses are good choice for the Fabry-Perot design. 
Four layer etalon
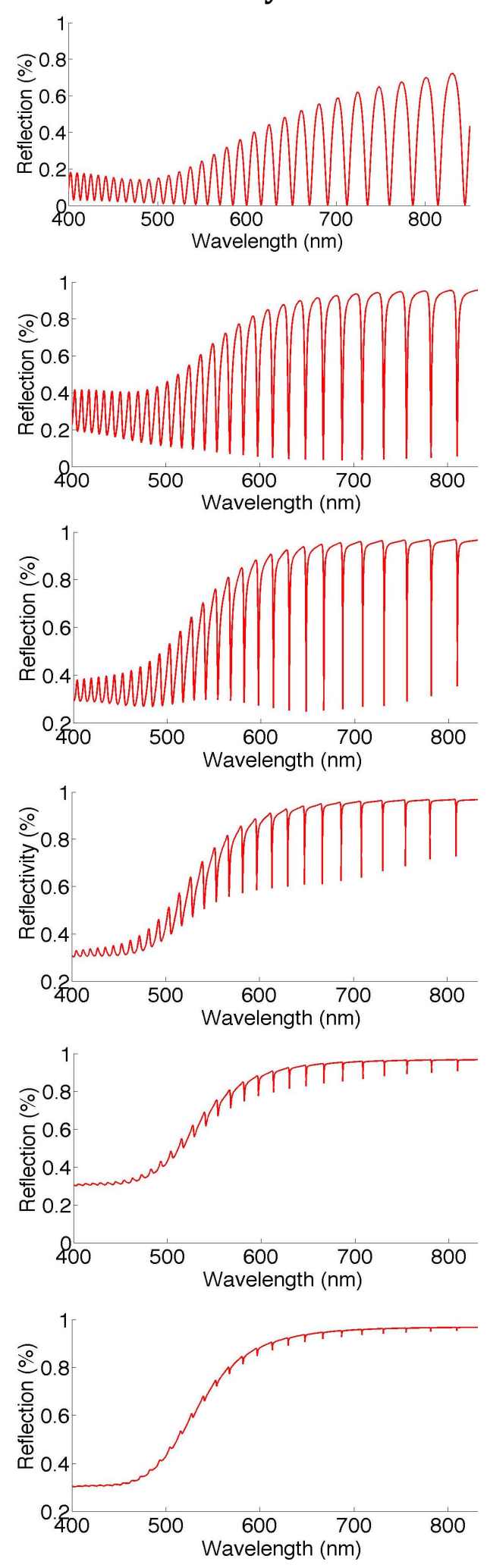

Five layer etalon

(a)

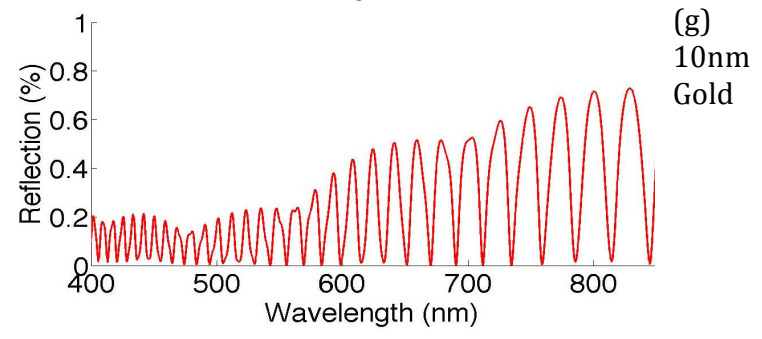

(b)

$30 \mathrm{~nm}$

Gold

(c)

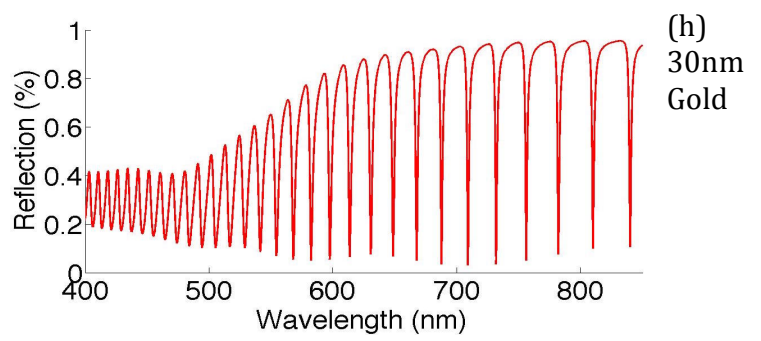

(i)

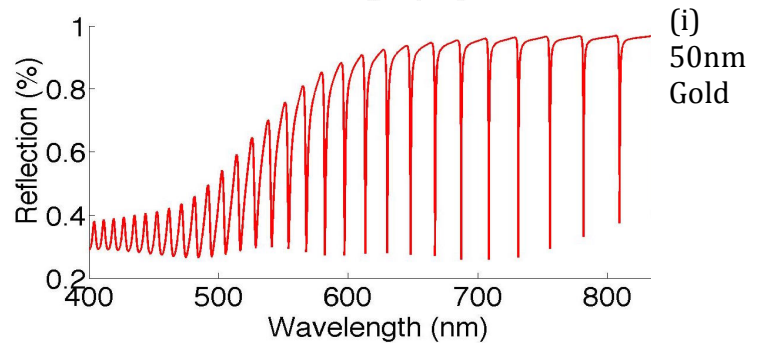

(d)

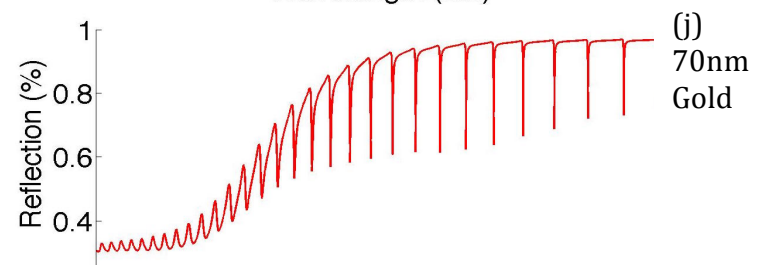

(e)

$90 \mathrm{~nm}$

Gold
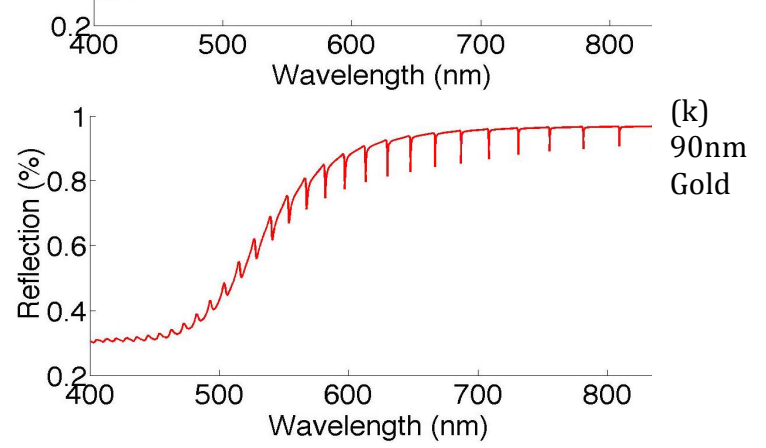

(f)

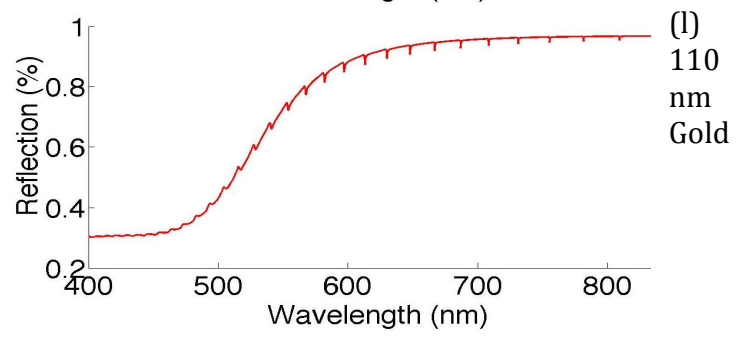

Figure 4.15 Simulation model of 'Reflection vs. wavelength' for nearly normal incidence light (p-polarized case) 


\subsection{REFLECTED LIGHT INTENSITY VS WAVELENGTH (NORMAL LIGHT INCIDENCE): EXPERIMENTAL RESULT}

We have measured the reflection spectrum for both four and five layer structures using a Shimadzu UV-3600 spectrometer. Figure 4.16 shows the results of these measurements.

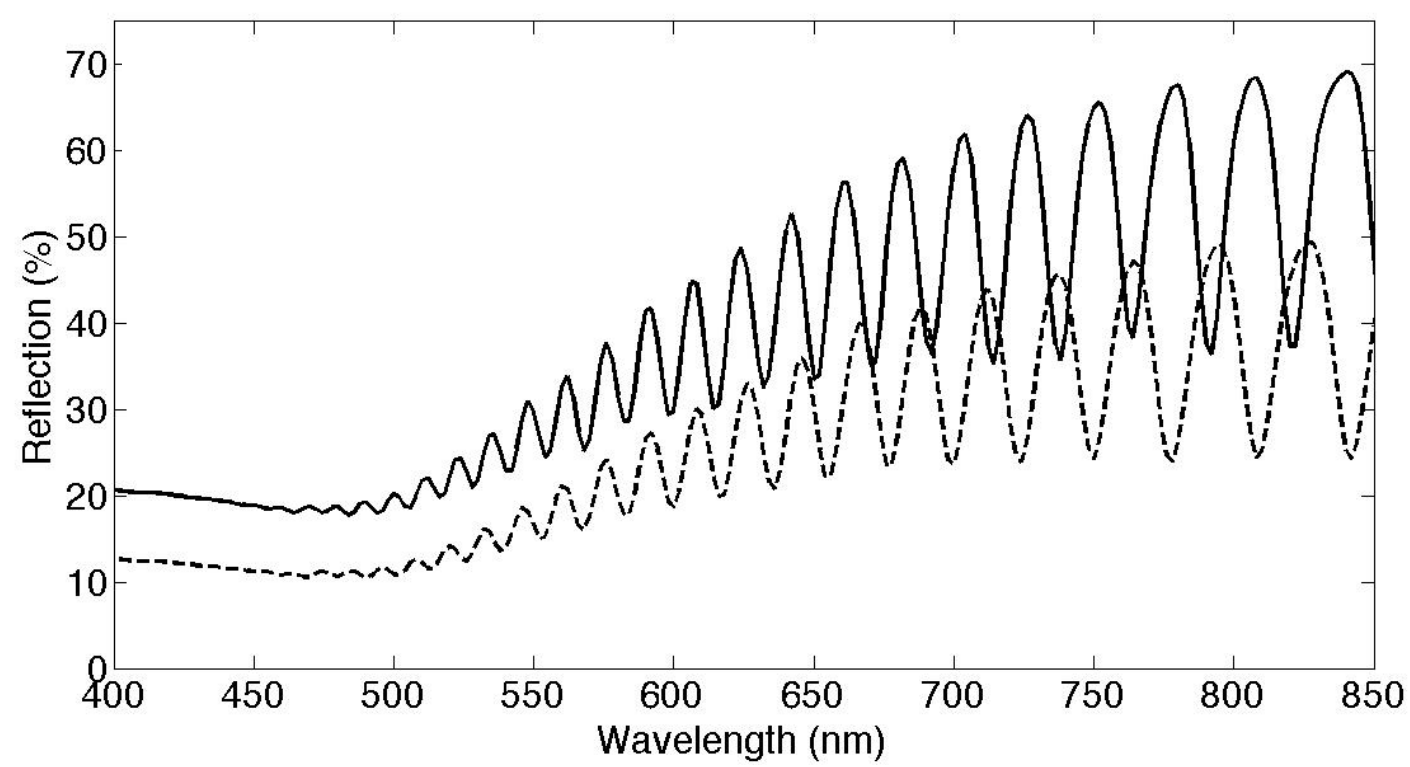

Figure 4.16 Reflectivity measurement of two types of Parylene etalons by spectrometer. The solid and dotted lines are representing the measurement results using five layer and four layer etalon respectively.

The spectrum has similar shapes for both cases, but the amplitude of reflection and position of the peak reflection are different. This is expected, since the five layer has a water protection layer, which the four later structure does not. As a result their optical characteristics are different.

Our five layer and four layer films were coated with gold for 160 and 180s respectively in a sputterer. Comparison of Figure 4.16 with those in Figure 4.15 shows that the former shows a good resemblance with Figure 15(d) and (j), which are the spectrum for $70 \mathrm{~nm}$ thick gold film. Therefore, we believe the gold thickness for the samples were close to $70 \mathrm{~nm}$. 


\subsection{GOLD THICKNESS MEASUREMENT}

\subsubsection{Resistance Measurement of All Films by Four-Probe Technique}

Using the 4-probe technique (section 3.7) we measured the resistance of some gold coated glass samples. The following table shows that measured resistance result of all the gold coated glass, coated for different times.

Table 4.3: Various samples, their time of gold coating, and their measured resistance values.

\begin{tabular}{|l|l|}
\hline $\begin{array}{c}\text { Time of gold } \\
\text { coating (s) }\end{array}$ & \multicolumn{1}{|c|}{$\begin{array}{c}\text { Experimentally } \\
\text { obtained film } \\
\text { resistance } \\
\text { (ohm/square) }\end{array}$} \\
\hline 300 & 6.43 \\
\hline 240 & 7.39 \\
\hline 220 & 8.05 \\
\hline 200 & 8.88 \\
\hline 180 & 9.5 \\
\hline 160 & 9.7 \\
\hline 140 & 12.5 \\
\hline 120 & 13.9 \\
\hline 100 & 13.4 \\
\hline 90 & 12.8 \\
\hline 80 & 12.2 \\
\hline 70 & 16.9 \\
\hline
\end{tabular}

The device did not show any stable resistance result for the samples coated for less than 70 seconds. 


\subsubsection{Reference Film Thickness Measurement by AFM Technique}
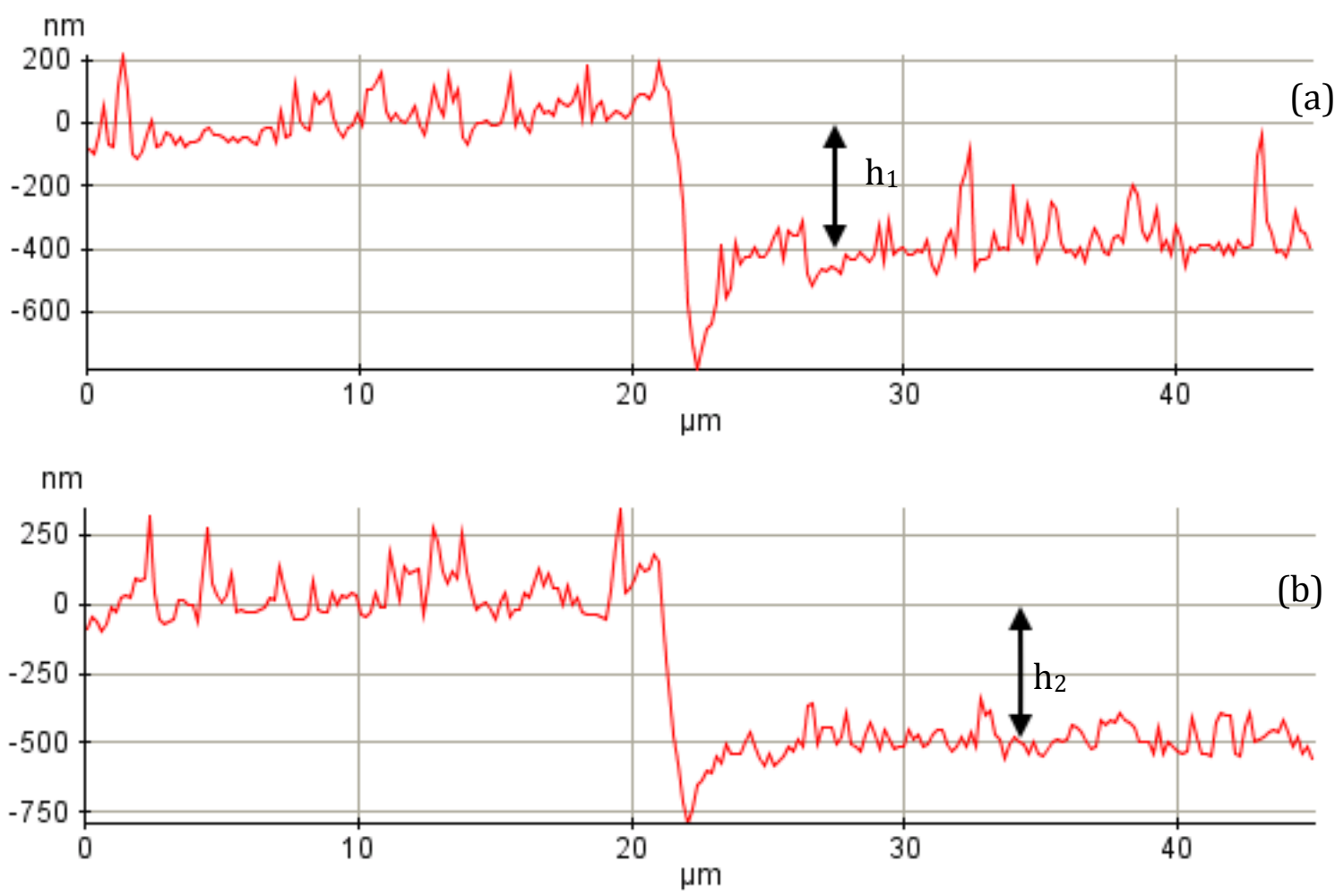

Figure 4.17 (a) A line profile on the scanning region 1 showing the height of glass substrate's upper surface to the bottom of rectangular laser pulse created hole $\left(\mathrm{h}_{1}=0 \mathrm{~nm}-(-\right.$ $400 \mathrm{~nm})=400 \mathrm{~nm}$ ). (b) Line profile of the scanning region 2 showing the height of gold layer's upper surface to the bottom of rectangular laser pulse created hole $\left(\mathrm{h}_{2}=0 \mathrm{~nm}-(-\right.$ $500 \mathrm{~nm})=500 \mathrm{~nm})$

Table 4.4 shows the calculated measurement of gold layer thickness with a reference film to normalize the data.

Table 4.4 Gold thickness measurement result of reference film (160s gold coating) using AFM device

\begin{tabular}{|l|l|}
\hline \multicolumn{1}{|c|}{ Range } & Depth $(\mathrm{nm})$ \\
\hline $\begin{array}{l}\text { 'Upper surface of glass to bottom' height } \\
\text { of the hole }\left(\mathrm{h}_{1}\right)\end{array}$ & 400 \\
\hline $\begin{array}{l}\text { 'Upper surface of gold to bottom' height } \\
\text { of the hole }\left(\mathrm{h}_{2}\right)\end{array}$ & 500 \\
\hline Obtained thickness of gold layer, $\mathrm{h}\left(\mathrm{h}_{2}-\mathrm{h}_{1}\right)$ & 100 \\
\hline
\end{tabular}




\subsubsection{Correlation between 'Gold Coating Time' and 'Gold Thickness'}

Using the reference gold thickness and the resistance result of Table 4.3, we obtained the gold thickness for all of the films. Here we plotted the measured gold thicknesses of all films vs their corresponding time of gold coating.

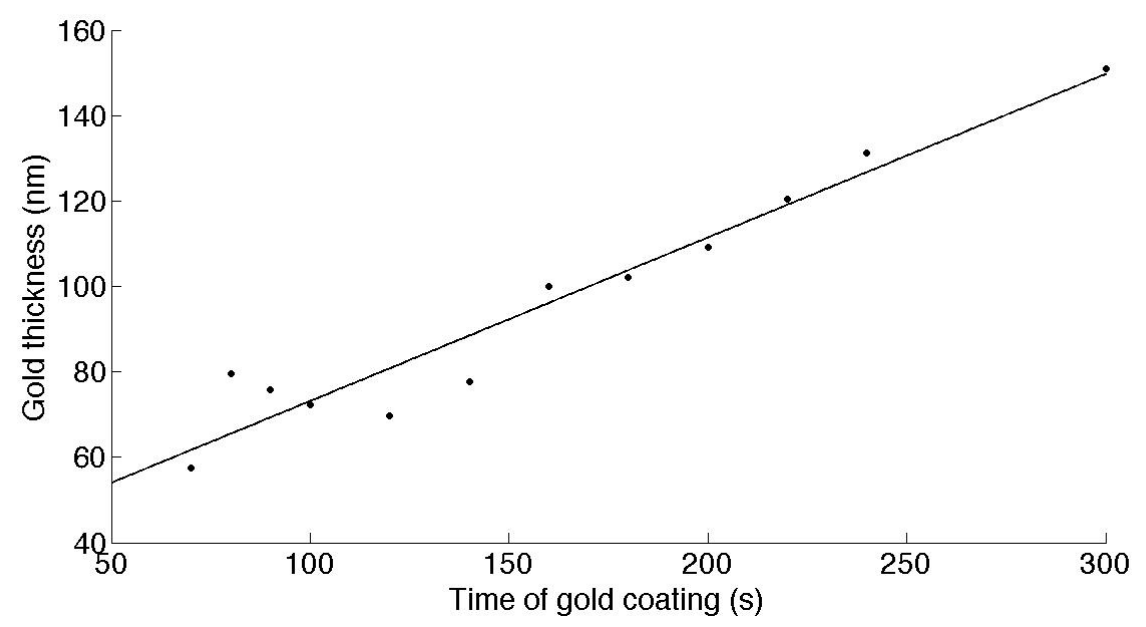

Figure 4.18 A graph showing a linear relationship between 'Gold coating time' and the measured 'Gold layer thickness'

In Figure 4.18 we see that the measured gold thicknesses deviated from linearity when the coating time was less than 150s. This was expected because the small gold particles are easily removable when they have a very thin thickness. This non-uniform thickness is more non-uniform compared to the thicker gold samples. For the same reason, we did not see a stable resistance measurement when using the four-probe device for the gold coated samples for coating times of less than 70 seconds. The gold layers (gold dusts) were too thin and almost gone from the substrates.

\subsubsection{Uncertainties In the Gold Thickness Measurement}

We prepared several gold samples, in which a layer of gold was deposited on glass and observed variations in sample thicknesses. The thickness of gold was not uniform on the substrate for each sample. We found, the thickness was largest in the middle of the substrate and smallest around substrate edge. The 'stage' of the sputter device was not a rotating stage, a rotating stage makes more uniform coating on substrates. We also 
observed that our samples had gold coating more like a layer of gold dust on substrate which comes off when it is dipped in water a few times or rubbed with a light touch. Measurement of the thickness of this kind of gold deposition is highly associated with measurement uncertainty.

There was another uncertainty in our gold thickness measurement. It was the inclined incident angle of the rectangular laser pulse. When a rectangular laser pulse is incident on any sample in an inclined angle, it makes 'rectangular well' with different height in its two sides, ie the bottom of this 'well' was not flat (Figure 4.19). We ignored this uncertainty in our measurement.

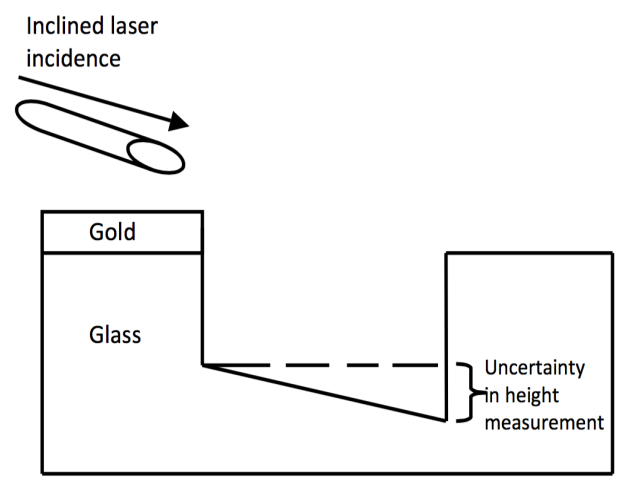

Figure 4.19 A diagram showing the measurement uncertainty due to the inclined incidence of laser

\subsection{OPTIMUM DESIGN FOR FP ETALON}

In the following sections, we describe our effort to find the optimum thickness of etalon, gold layer thickness and wavelength range of the Fabry-Pero.

\subsubsection{Optimum Thickness of Etalon}

The theory of section 2.4.2 (Equation 2.33) gave us the graphical result of Figure 4.20. Figure 4.20 describes that at $541.9 \mu \mathrm{m}$ etalon thickness, the maximum etalon thickness change can be observed. 


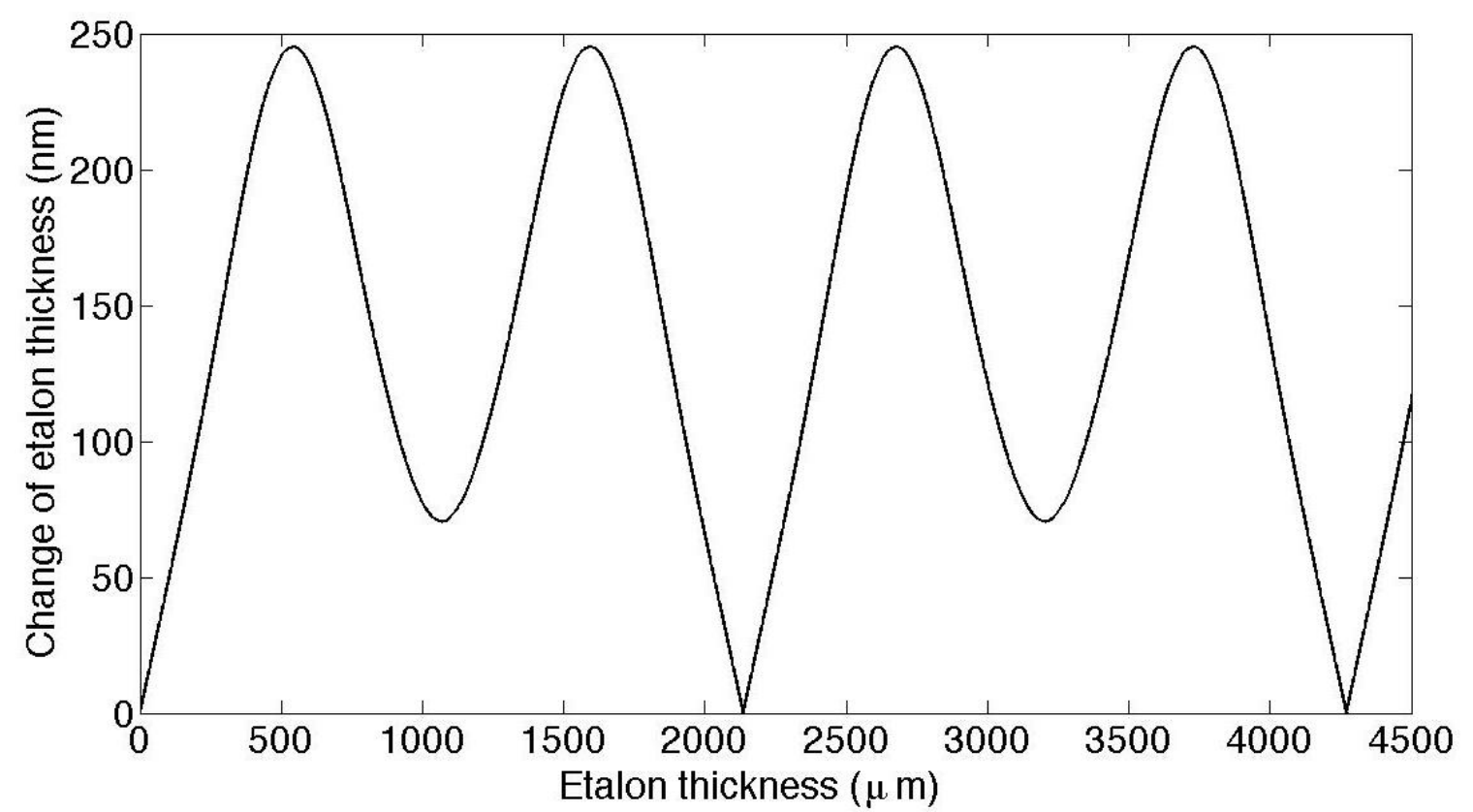

Figure 4.20 Change of etalon thickness vs etalon thickness graph for Parylene considering the reflectivity of the etalon as 0.9 (ultrasound frequency $1 \mathrm{MHz}$ ).

There is a minima (first minima) found in this graph at thickness $2135 \mu \mathrm{m}$. This is actually the considered velocity of ultrasound in Parylene media. This point corresponds a full wavelength of ultrasound in Parylene media. The thickness $541.9 \mu \mathrm{m}$ is $\sim 1 / 4^{\text {th }}$ wavelength of ultrasound in Parylene media. At this thickness, the etalon thickness change found maximum. So, for optimization, we should avoid choosing a thickness of etalon which is equal to the wavelength of ultrasound in that media, and we can get a maximum thickness change when the thickness is $\sim 1 / 4^{\text {th }}$ of ultrasound wavelength in Parylene etalon media.

\subsubsection{Optimum Thickness of Gold Layer}

A first derivative of any function signifies a slope of a graph at any point. We are interested to know which thickness of gold is capable of producing the maximum reflectivity change. An interferometer film is called most sensitive when a pressure exposure causes a maximum output intensity change. Gold reflectivity is changed with its thickness but also it is a function of light wavelength. In this section we will find the gold thickness necessary to make the most sensitive interferometer and also a suitable wavelength range. 
The way [19] we followed to find the optimum thickness of gold was first to find the 'Reflection coefficient vs the wavelength' graph for the optimum thickness of etalon. Then from this graph, we plotted another graph, the 'First derivative of reflection coefficient vs wavelength'. This was done for the gold thickness of $0.0 \mathrm{~nm}$ to $100 \mathrm{~nm}$. For each gold thickness we recorded the maximum derivative then finally plotted 'Max derivative vs Gold thickness' graph. This graph had bell shape and the gold thickness corresponding to the peak point of this 'bell' gave us the optimum thickness of gold. Figure 4.21 and Figure 4.22 showing the results obtained in this way.
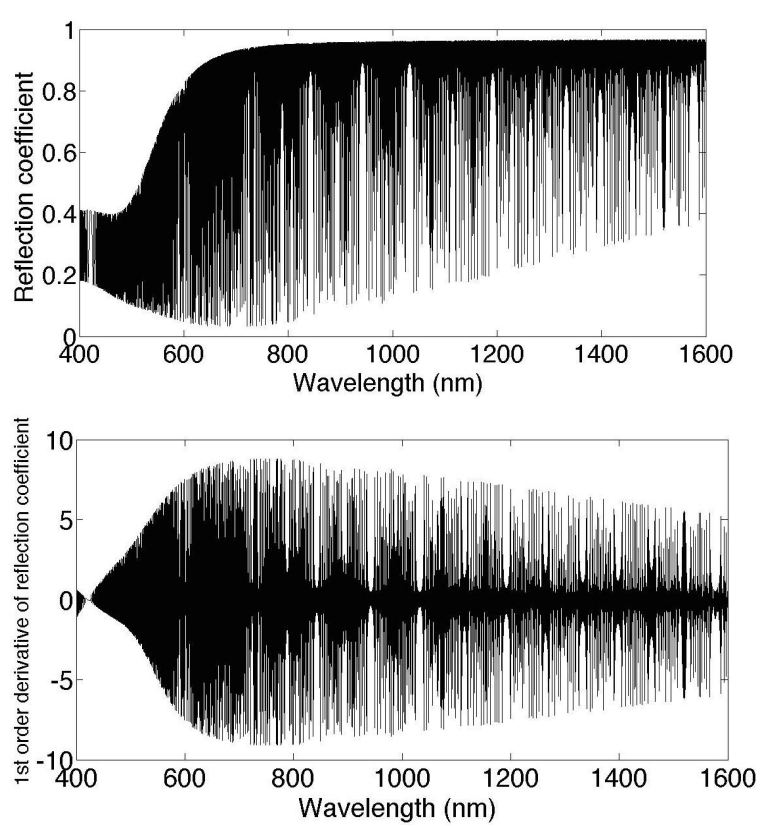

(a)

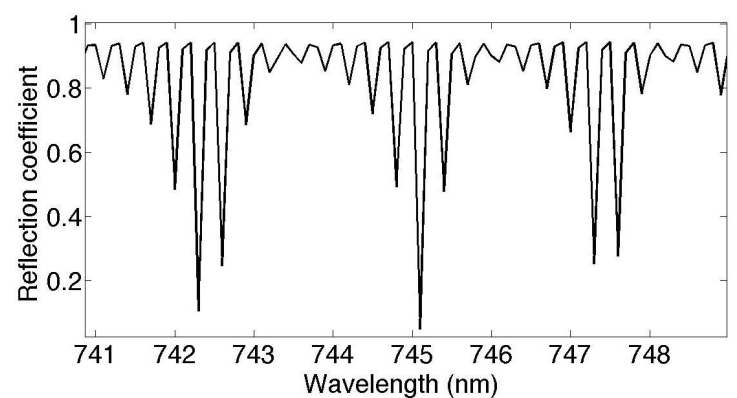

(b)

(c)

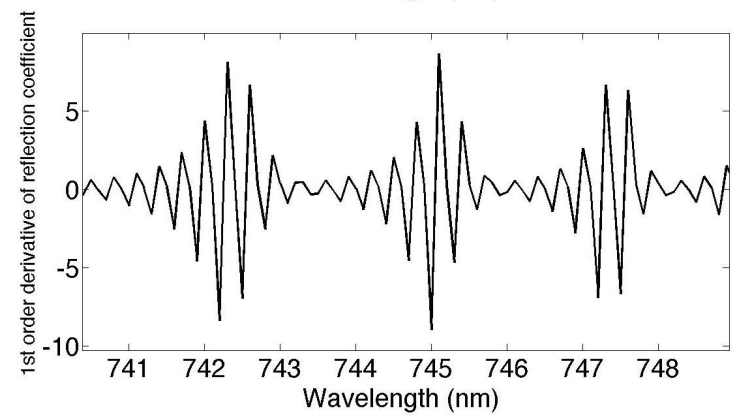

Figure 4.21 Sample plots for $29 \mathrm{~nm}$ gold thickness: (a) Reflection coefficient vs wavelength graph for Parylene film interferometer. (b) Zoom in view of (a); (c) First derivative of reflection coefficient vs wavelength graph; (d) Zoom in view of (c). 


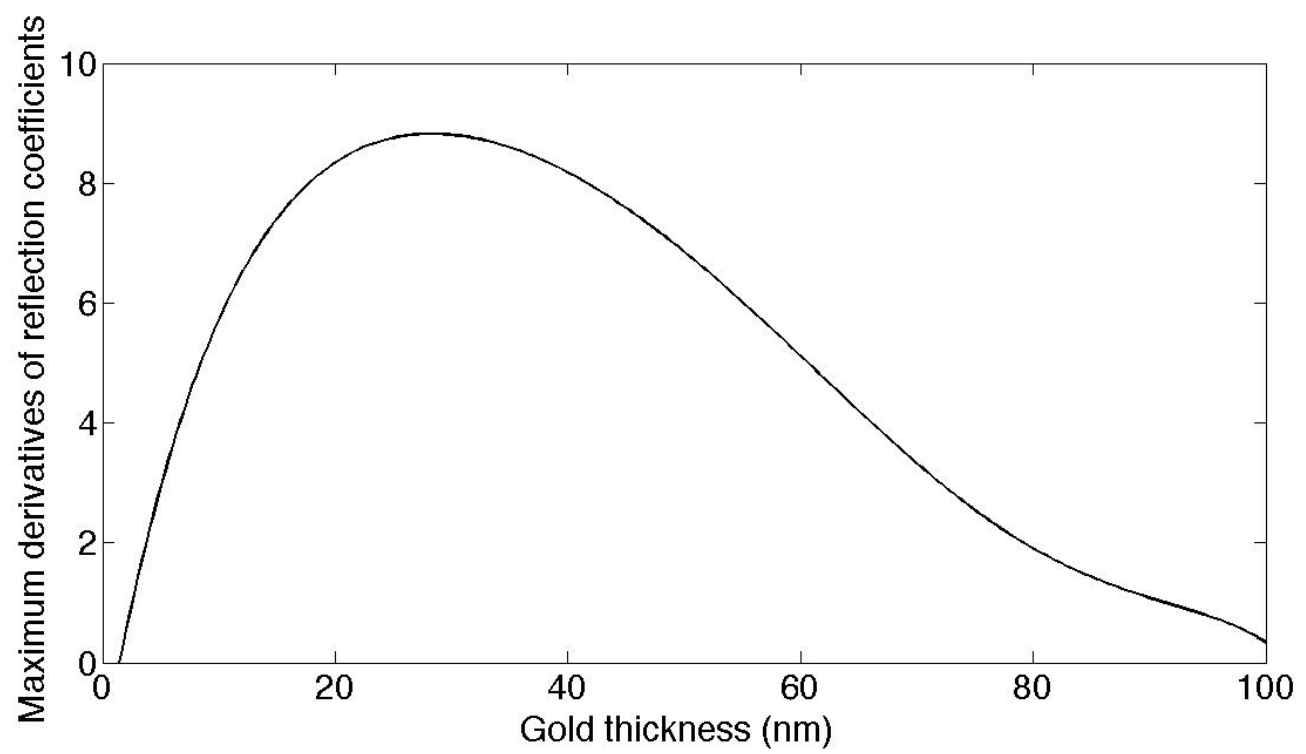

Figure 4.22 Maximum derivative of reflection coefficients vs Gold thickness graph, plotted for $541.9 \mu \mathrm{m}$ Parylene film.

We can present the result of Figure 4.22 numerically in Table 4.5

Table 4.5 Table for polymer thickness, gold thickness, and maximum derivative

\begin{tabular}{|l|l|l|}
\hline $\begin{array}{c}\text { Polymer } \\
\text { Thickness } \\
(\mu \mathrm{m})\end{array}$ & $\begin{array}{c}\text { Gold } \\
\text { Thickness } \\
(\mathrm{nm})\end{array}$ & \multicolumn{1}{|c|}{$\begin{array}{c}\text { Maximum } \\
\text { Derivative }\end{array}$} \\
\hline 541.9 & 29 & 8.801 \\
\hline 500 & 28 & 8.747 \\
\hline 400 & 30 & 8.600 \\
\hline 300 & 32 & 8.365 \\
\hline 200 & 35 & 7.959 \\
\hline 100 & 40 & 6.961 \\
\hline 50 & 45 & 5.459 \\
\hline 25 & 49 & 3.700 \\
\hline 10 & 51 & 1.745 \\
\hline 5 & 52 & 0.908 \\
\hline
\end{tabular}

Here we see, we obtained a maximum derivative value for a polymer film thickness of $541.9 \mu \mathrm{m}$ and a $29 \mathrm{~nm}$ gold coating on each side of this polymer film. 


\subsubsection{Optimum Wavelength Range}

For a particular gold thickness, we found the 'Reflection coefficient vs the wavelength' graph for the optimum thickness of etalon (Figure 4.21 (a),(b)). Then from this graph, we plotted another graph, the 'First derivative of reflection coefficient vs wavelength' (Fig 4.21 (c),(d)). We here recorded the associated wavelength of maximum derivative of this plot. Now, this process was done for the gold thickness of $0.0 \mathrm{~nm}$ to $100 \mathrm{~nm}$. For each gold thickness we recorded the wavelength associated the maximum derivative and then plotted 'Optimum wavelength vs Gold thickness' graph (Figure 4.23).

We obtained Figure 4.23 as the result of finding the optimum wavelength range.

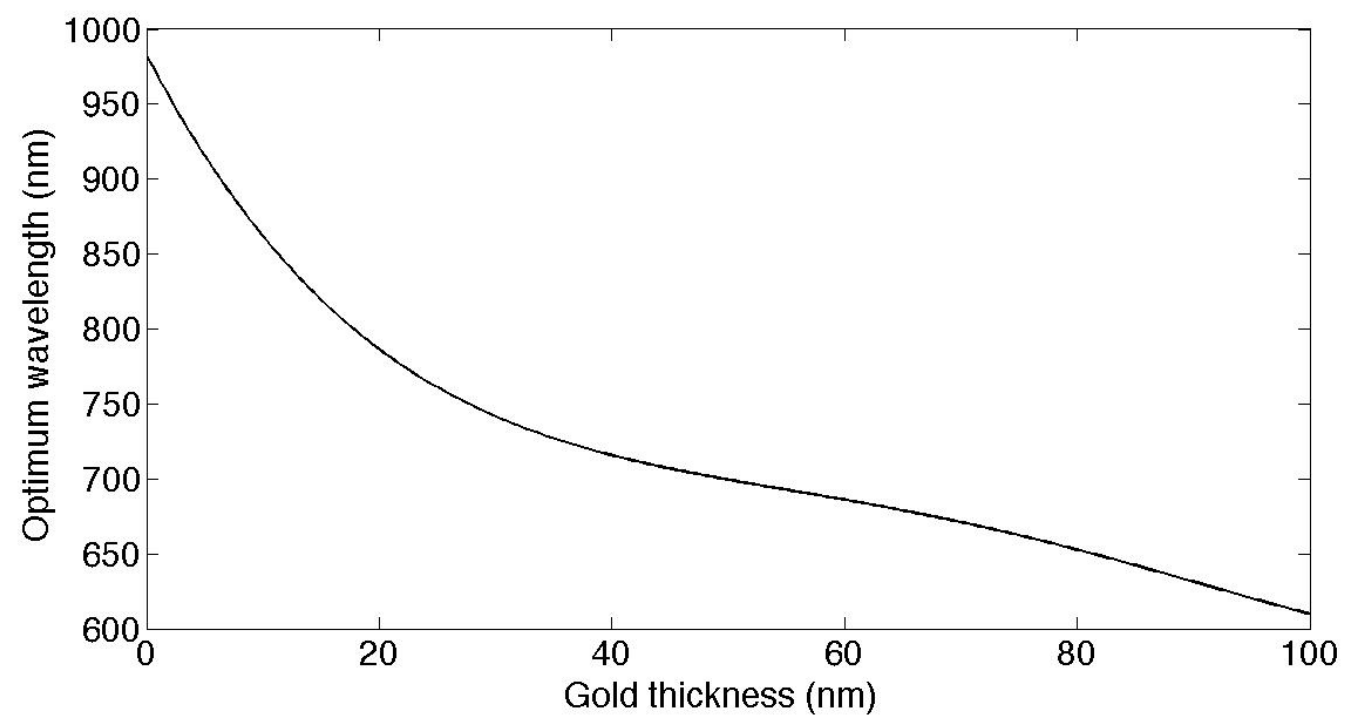

Figure 4.23 Optimum wavelength vs Gold thickness graph showing the optimum wavelength range $(600 \mathrm{~nm}-1000 \mathrm{~nm})$ plotted for $541900 \mathrm{~nm}$ thick, and 0.9 reflectivity Parylene film interferometer.

The graphical result shows us a suitable wavelength range $(600 \mathrm{~nm}-1000 \mathrm{~nm})$ to perform this experiment best. This result also agreed with the gold reflectivity properties, as in this wavelength range gold shows a better reflectivity. Here, the wavelength corresponding to $29 \mathrm{~nm}$ gold thickness found as $745.4 \mathrm{~nm}$.

Table 4.6 representing the final result of this section, the obtained polymer thickness, gold thickness, and wavelength range of our optimization. 
Table 4.6 Final optimization results for a Fabry-Perot device

\begin{tabular}{|l|l|}
\hline \multicolumn{1}{|c|}{ Parameters } & \multicolumn{1}{c|}{ Result } \\
\hline Polymer thickness & $541.9 \mu \mathrm{m}$ \\
\hline Gold layer thickness & $29 \mathrm{~nm}$ \\
\hline Optical wavelength range & $(600 \mathrm{~nm}-1000 \mathrm{~nm})$ \\
\hline Wavelength at optimum gold thickness & $745.4 \mathrm{~nm}$ \\
\hline
\end{tabular}




\section{Chapter 5 DISCUSSION, CONCLUSION AND FUTURE WORK}

\subsection{DISCUSSION}

In this thesis our main goal was the successful detection of lower frequency ultrasound using our proposed optical method. From our experiment, we realized that the success of our experiment mainly depends on the prepared etalon's quality. The etalon quality is related to the appropriate gold thickness and a good gold deposition process. The etalons we designed and constructed were capable of detecting ultrasound in our laboratories. For the medical application of this technique, we must ensure the high quality and stability of the etalons.

An etalon performance depends on the light wavelength. Usually a tunable continuous laser is needed to set the etalon at the best performance point. But, this type of laser was not available in our labs. So the dependence of the etalon performance on the light wavelength was demonstrated indirectly by measuring the signal at various light incidence angles. This was possible because the interferometer output is a function of light wavelength and light incidence angle in the form of phase difference $\delta$ only.

In our experimental arrangement we used a low noise current pre-amplifier which was an essential device for our circuit. Without this device our optical detection was not working. There was another essential parameter to get our optical signals. It was a 'minimum averaging number' of the output signals in oscilloscope. In our result chapters we averaged the signal sometimes 4096 times or sometimes 1024 times. We did a separate experiment to detect the minimum averaging number to find out the optical signal without excess averaging. In that experiment we choose an optical signal that was 
employed to detect an ultrasound signal. Keeping the gain, pulser voltage and other parameters constant, we changed its averaging number from maximum to minimum (averaging number according to the oscilloscope availability). From the result of this experiment, we observed, how the SNR vary with the averaging number. We obtained the graph of Figure 5.1(a).
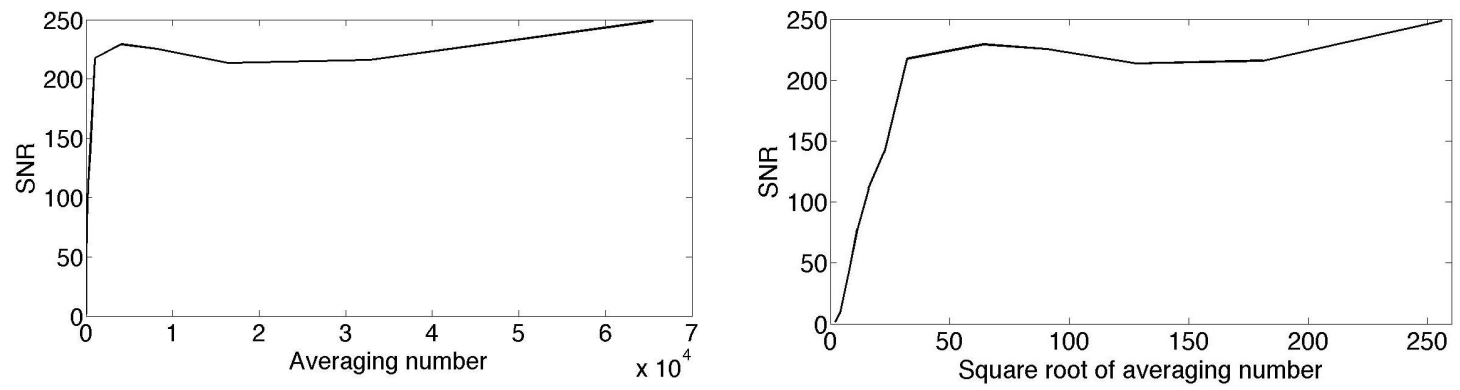

Figure 5.1 (a) SNR vs. averaging number plot (b) SNR vs. square root of averaging number plot

Figure 5.1 (a) shows that, the SNR increased linearly until a certain averaging number (1024). Averaging more than 1024 times will also give us high SNR, but we should use a reasonable number for averaging. Figure 5.1(b) shows a plot of SNR vs square root of averaging number. We here see, until a certain number there is a linear region. After that the curve goes to saturation. Both the linear and saturation region were expected to us. We also found that when the averaging number is less than the averaging number 16 , the optical signal was no longer seen (undetectable from noise).

We performed the experiment relating the reflected light intensity and incident angle. We used a Goniometer for the fractional measurement of the incidence angle, but for this type of experiment, a Goniometer is not a perfect device for fractional angle measurement. A more precise device for measuring angles (like .1 or .01 degree) could help us to get a better result.

Getting uniform gold coating and measuring its thickness were challenging. The gold coating system that we used was unable to make a uniform coating on the substrate. We observed that the gold coating was thickest at the center of the substrate and minimum around the substrate edge. The stage of the sputter device where we kept the substrates was a stationary stage, but the stage that rotates, makes more uniform deposition on 
substrate. We also observed that the gold coating was not stable on the substrates. The gold coating system is an important issue; it should be better developed. The coating should be uniform and stable as gold property changes with the thickness in nm level. Laser incidence angle was another major uncertainty in our gold thickness measurement experiment. Avoiding the uncertainties could give us a better agreement between theory and experiment.

Although our experimentally obtained "Reflected light intensity vs light incidence angle" (Figure 4.13) result did not match well with our simulation results (Figure 4.9, Figure 4.10), we took a further step to find a match between the normalized "Reflected light intensity vs light incidence angle" and our model. We divide the Y-axis values of our result by $\cos ^{2} \theta$ and obtained the following result.

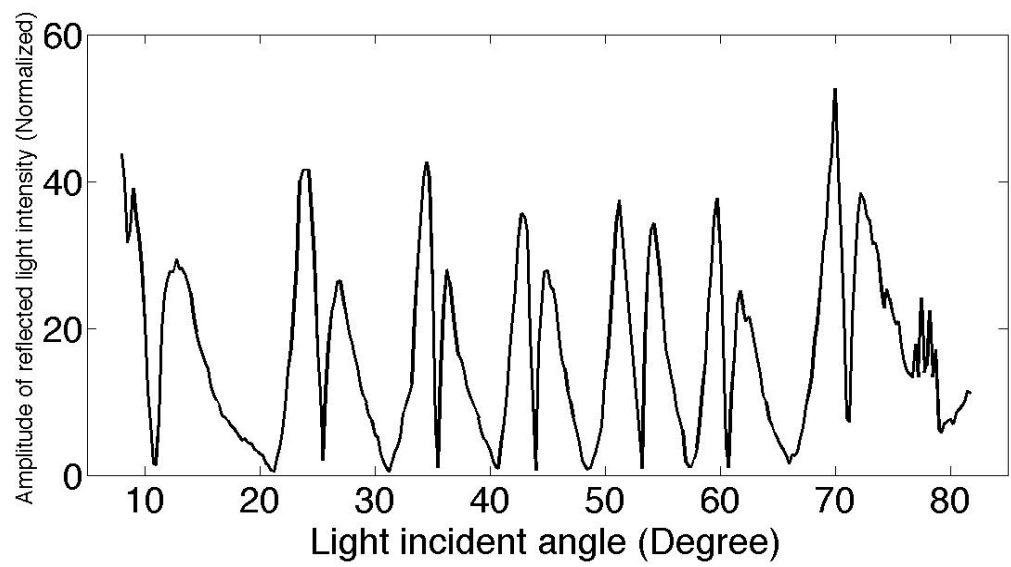

Figure 5.2 Normalized plot of reflected light intensity vs light incident angle.

The normalized plot has much more similarity with our simulation results than the unnormalized result as we obtained almost same amplitude for all the peaks. It means that amplitude of our original result reduces as $\cos ^{2} \theta$. The effect of the incidence angle on the acoustic sensitivity of the etalon might partially explain this factor.

We observed during our regular experiments that, when a transducer is submerged in water and is used to generate an ultrasound signal in water. A laser from outside whose beam transverses the path of the ultrasound propagation was modulated strongly after passing the ultrasound beam. When laser light passes through the ultrasound pressure 
variations in water, the light is modulated by the ultrasound field. This was clearly observed in the oscilloscope monitor when the light beam is captured by a diode. This is likely due to the changes in the index of refraction as the pressure waves intersect the laser beam. We made a simple experimental arrangement to study our observation. The experimental arrangement is shown in Figure 5.3
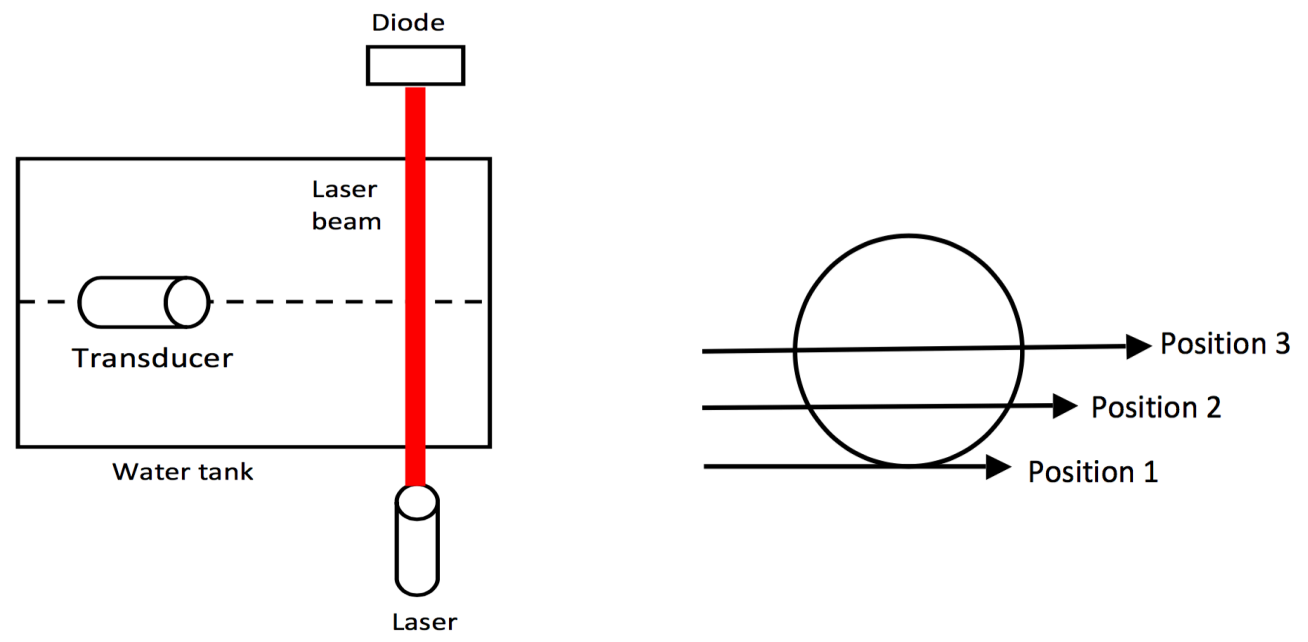

Figure 5.3 (a) A view from the top of our experimental arrangement (b) Circular transducer surface and lines representing the laser beam paths. The line 'Position 1' corresponds to a laser beam path going through the edge of circular transducer surface. 'Position 3' corresponds to a laser beam path along a line through the diameter of the circular surface. 'Position 2' corresponding a laser beam path between positions 1 and 3 .

In our arrangement, we fixed the laser tightly in its position, which was set outside the tank, but we moved the transducer along its direction in water (the dotted line of Figure 5.3(a) showing the direction of transducer movement). The transducer axis and laser beam direction were perpendicular to each other. We first kept the transducer surface in a minimum distance with the laser beam $(0 \mathrm{~cm})$. The beam level was carefully set to any level of position 1, 2, or 3 (Figure 5.3(b)). Now we set the diode in the opposite side of the laser, situated outside of the tank to capture the whole laser beam coming through water. When the transducer was switched on, we measured a strong modulation of the optical signal on our oscilloscope monitor and we recorded the diode signal strength. After first measurement, we increased the 'transducer surface-laser beam' distance by half centimeter, moving only the transducer and captured the laser beam for this new position. We continued recording the optical signals strength for each transducer surface- 
laser beam distance. We varied the distance from $0 \mathrm{~cm}$ to $14.5 \mathrm{~cm}$ and used a $1 \mathrm{MHz}$ transducer with focal length $\sim 4 \mathrm{~cm}$ and diameter $2.54 \mathrm{~cm}$. We plotted 'Optical signal amplitude vs transducer surface and light beam distance' and obtained the following result (Figure $5.4(\mathrm{a}))$.
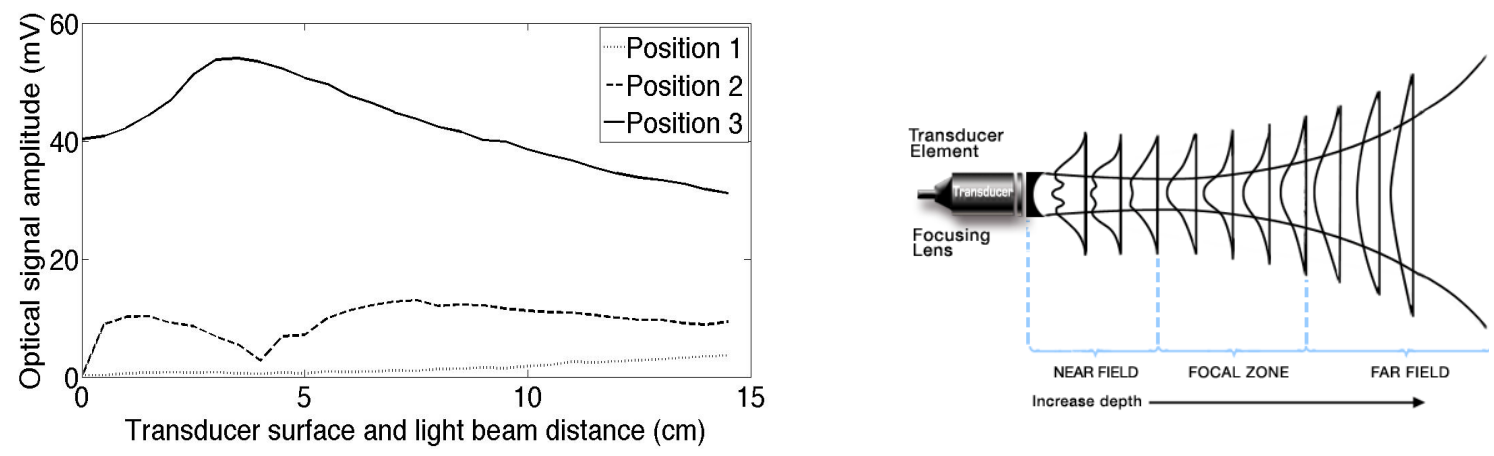

Figure 5.4 (a) Graphical result of Optical signal amplitude vs transducer surface and light beam distance (b) A general sound intensity profile of a focused transducer [45].

The optical signals were very weak near the transducer surface, but increased as the transducer and light beam distance was increased. The change in optical signal intensity was consistent with the theoretical axial pressure profile of the ultrasound beam. The expected beam intensity profile of a focused transducer is shown in Figure 5.4(b). The light beam gradually entered in ultrasound field. For light beam in position 2, we saw there was a weaker signal. For the light beam in position 3, we saw a strong optical signal amplitude, which was strongest at focal distance. This was because the beam pressure was high at the axial area and maximum at focal point. From this result we can comment the curve shape for the three position agrees with our expectation.

\subsection{CONCLUSION}

Our experiment established that we can detect lower frequency ultrasound accurately $(0.5$ $\mathrm{MHz}$ or $1 \mathrm{MHz}$ ) using our proposed optical method. We determined a linear relationship between the strength of an optical signals and the exerted pressure on a film by the ultrasound. 
We performed some modeling work of reflected light intensity variation with incidence angle. Using a theoretical transformation of this graph we obtained a graphical result of the reflected light intensity variation with wavelength. Both graphical results had the same shape, and a set of peaks with decreasing amplitude as the wavelength increases. The main point of this transformation was to demonstrate indirectly that the amplitude of the ultrasound modulated signals strongly depends on the light wavelength. An optimized wavelength can enhance the signals significantly.

In our optimization work, we tried to optimize a design for Parylene (or Parylene-C type polymer, like: SU8) etalon considering a high reflectivity 0.9 , for $1 \mathrm{MHz}$ frequency ultrasound. Our modeling result indicated that a $541.9 \mu \mathrm{m}$ thickness of Parylene-C polymer and a $29 \mathrm{~nm}$ gold thickness on each side of the polymer can give us the highest sensitivity. Also the optimized wavelength range should be $600 \mathrm{~nm}$ to $1000 \mathrm{~nm}$.

\subsection{FUTURE WORK}

We optimized a design for a Fabry-Perot device, but more analysis is needed on this optimization result, such as considering the effect of the substrate material on the etalon thickness optimization. After a better analysis we can fabricate the design and can test its performance as a sensor for ultrasound detection.

Our proposed optical method has a potential for 2D and 3D ultrasound imaging. The Beard group [16] was already successful in 2D mapping of an ultrasound field by using a pulse laser and a CCD camera. Their experiment can be extended to ultrasound detection with a high speed camera in our future study. In this case, it is important to study if the camera is sensitive enough to detect the ultrasound induced light change when the exposure time is short, smaller than 1 microsecond, and the imaging frame is at $\mathrm{MHz}$ range. 
Our experiment of reflected light intensity variation with oblique light incidence angle was done with unpolarized light. We can do this experiment while controlling the polarization. Also using a better set-up with a precise angle measurement system, this experiment can be improved in the future.

This technique of ultrasound detection is investigated to use in medical sector. An etalon, suitably made and characterized for medical application, can be used on patients. Placing the etalon on patients' skin, we can send ultrasound on patients' skin from outside of his body, and from the same side of the etalon. The ultrasound will be reflected from the internal tissues. The reflected ultrasounds will create a mechanical pressure on the etalon and thickness of it will be changed due to this mechanical pressure. A laser incident on the etalon will be reflected from the two highly reflecting surfaces of the etalon and the reflected output intensity is a measureable property in this technique. The intensity of the reflected beam will be changed according to the thickness change of the etalon, which is a function of the ultrasound pressure. For medical application, the etalon must be well calibrated to obtain the pressure amplitude information of ultrasound from the output beam of reflected light intensity.

The experiment we did in Figure 5.3 can form the basis of another method of ultrasound detection for the future students as the optical signals we obtained were very strong and easily detectable [46]. 


\section{REFERENCES}

[1] Y. Hou, S.-W. Huang, S. Ashkenazi, R. Witte, and M. O'Donnell, “Thin polymer etalon arrays for high-resolution photoacoustic imaging", submitted to IEEE Trans. Ultrason. Ferroelect. Freq. Contr. (2008).

[2] Y. Hou, "Broadband all-optical ultrasound transducers for high-resolution ultrasound imaging", $\mathrm{PhD}$ thesis, University of Michigan (2008)

[3] F. M. Abu-Zidan, A. F. Hefny, and P. Corr, "Clinical ultrasound physics.," J. Emerg. Trauma. Shock, vol. 4, no. 4, pp. 501-503, Oct. 2011.

[4] T. Szabo, Diagnostic Ultrasound Imaging Inside Out. Saint Louis, MO, USA: Elsevier, Academic Press, 092004.

[5] K. Dussik, "On the possibility of using ultrasound waves as a diagnostic aid," Neurology and Psy- chiatry, vol. 174, pp. 153-168., 1942.

[6] P. Laugier and H. Guillaume, Bone quantitative ultrasound. Springer New York, 2011.

[7] H. Varu, "The optical modeling and design of Fabry Perot Interferometer sensors for ultrasound detection", PhD Thesis, University College, London (2014)

[8] Beard, PC, and Mills, TN (2000): An optical detection system for biomedical photoacoustic imaging, Proc. SPIE 3916, 100-109.

[9] S. Ashkenazi, Y. Hou, T. Buma and M. O'Donnell, "Optoacoustic imaging using thin polymer etalon”, Appl. Phys. Lett. 86, pp. 134102-1-3 (2005)

[10] Beard, PC, Perennes, F, and Mills, TN (1999): Transduction mechanisms of the Fabry Perot polymer film sensing concept for wideband ultrasound detection, IEEE Transactions on Ultrasonics, Ferroelectrics and Frequency control 46(6), 1575-1582

[11] P. C. Beard and T. N. Mills, "Extrinsic optical fibre ultrasound sensor using a thin polymer film as a low finesse Fabry-Perot interferometer," Appl. Opt., vol. 35, no. 4, pp. 663-675, 1996

[12] Zhang, E, Laufer, J, Beard, P (2008): Backward-mode multiwavelength photoacoustic scanner using a planar Fabry-Perot polymer film ultrasound sensor for high-resolution three-dimensional imaging of biological tissues, Applied Optics 47, 561577.

[13] Beard, PC, Hurrell, A, and Mills, TN (2000): Characterisation of a polymer film optical fibre hydrophone for the measurement of ultrasound fields for use in the range 130MHz: a comparison with [ PVDF needle and membrane hydrophones, IEEE Transactions on Ultrasonics, Ferroelectrics and Frequency control 47(1), 256-264. [14] J. D. Hamilton, "High frequency optoacoustic arrays using etalon detecton" [15] Beard, PC, and Mills, TN (2000): A 2D optical ultrasound array using a polymer film sensing interferometer, Proc. IEEE Ultrasonics Symposium, 1183-1186.

[16] Lamont, M, and Beard, PC (2006): 2D imaging of ultrasound fields using a CCD array to detect the output of a Fabry Perot polymer film sensor, Electronics Letters 42(3), 187-189. 
[17] S. Ashkenazi, Y. Hou, S.-W. Huang, T. Buma, and M. O’Donnell, "High frequency optoacoustic transducers for ultrasonic and photoacoustic imaging", in Photoacoustic imaging ed. L. V. Wang (invited book chapter)

[18] S. Ashkenazi, Y. Hou, S.-W. Huang, T. Buma, and M. O’Donnell, "High frequency optoacoustic transducers for ultrasonic and photoacoustic imaging", in Photoacoustic imaging ed. L. V. Wang (invited book chapter)

[19] Y. Hou, S. Ashkenazi, and M. O'Donnell, "Improvements in Optical Generation of High Frequency Ultrasound", IEEE Trans. Ultrason. Ferroelect. Freq. Contr. 54, (3) pp. 682-686 (2007)

[20] Y. Hou, J. S. Kim, S. Ashkenazi, M. O’Donnell, and L. J. Guo, “Optical generation of high frequency ultrasound using two-dimensional gold nanostructure", Appl. Phys. Lett. 89, pp. 093901 (2006)

[21] X. Guo, "High frequency ultrasound transducer for real time ultrasound biomicroscopy with optoacoustic arrays", Masters thesis, University of Delaware (2011)

[22] E. Hect, A. R. Ganeshan, "Optics", $4^{\text {th }}$ Ed.

[23]

http://ujdigispace.uj.ac.za/bitstream/handle/10210/2170/CHAPTER4.pdf?sequence=9

January 2014

[24] https://en.wikipedia.org/wiki/Fabry\%E2\%80\%93P\%C3\%A9rot_interferometer January 2014

[25] Beard, PC (2003): Interrogation of Fabry Perot sensing interferometers by angle tuning, Measurement Science and Technology 14, 1998-2005.

[26] Zhang, EZ, Cox, BT, and Beard, PC (2004): Ultra high sensitivity, wideband Fabry Perot ultrasound sensors as an alternative to piezoelectric PVDF transducers for biomedical photoacoustic detection, Proc. SPIE 5320, 222-229.

[27] http://paperity.org/p/4022989/the-optical-properties-of-gold January 2014

[28] http://microchem.com/pdf/SU8_2-25.pdf September 2014

[29] http://memscyclopedia.org/su8.html September 2014

[30] http://www.microchem.com/pdf/SU-82000DataSheet2000_5thru2015Ver4.pdf

September 2014

[31]

http://www.nbtc.cornell.edu/sites/default/files/Parylene\%20Information\%20Sheets.pdf September 2014

[32] http://vp-scientific.com/parylene_properties.htm September 2014

[33] https://www.fishersci.com/shop/products/fisherbrand-superfrost-plus-microscopeslides-2/p-45174\#tab2 January 2014

[34] http://www.lamp.umd.edu/Sop/Piranha_SOP.htm September 2014

[35] S. Franssila, Introduction to Microfabrication, John Wiley and Sons 2010.

[36] http://www.cleanroom.byu.edu/su8.phtml September 2014

[37] https://www.utdallas.edu/ rar011300/Parylene/ParyleneDepManual.pdf September 2014

[38] www.adahlin.com January 2014

[39]http://www.plexiglas.com/export/sites/plexiglas/.content/medias/downloads/sheetdocs/plexiglas-general-information-and-physical-properties.pdf September 2016 [40] https://en.wikipedia.org/wiki/File:Water_reflectivity.jpg September 2016

[41] http://web.tuat.ac.jp/ usuilab/English/depo.html September 2014

[42] http://nano.indiana.edu/index.php/instrument/parylene-coater/ September 2014 
[43] https://www.alibaba.com/product-detail/WS-650-23NPP-SpinCoater_109189784.html September 2014

[44] http://sharedresources.asu.edu/resources/5077\# September 2013

[45] https://www.alibaba.com/product-detail/WS-650-23NPP-Spin-

Coater_109189784.html September 2014

[46] http://iopscience.iop.org/article/10.1088/1742-6596/214/1/012009 April 2017

[47] M. Suter and P. Dietiker, Calculation of the finesse of an ideal Fabry-Perot resonator, Applied Optics 53 (30): 7004-7010 (2014).

[48] S. Moten, Modeling of an ultrasonic transducer for cardiac imaging, Traineeship

Report, Eindhoven University of Technology 2010.

[49] http://www.ondacorp.com/products_hydrophones.shtml January 2014

[50] M. Kolios, "Biomedical ultrasound imaging from 1 to $1000 \mathrm{MHz}$ "

[51] Pedrotti, Pedrotti, Pedrotti, 'Introduction to Optics', $3^{\text {rd }}$ Ed.

[52] Ph. Pre tre, L.-M. Wu, R. A. Hill, and A. Knoesen:

Characterization of electro-optic polymer films by use of decal-deposited reflection Fabry-Perot microcavities, Vol. 15, No. 1/January 1998/J. Opt. Soc. Am. B

[53] H. H. Nguyen. J. Park, S. Kang, and M. Kim, "Surface plasmon resonance: A versatile technique for biosensor applications," Sensors 15(5): 10481-10510 (2015)

[54] B. Dong, C. Sun, and H. F. Zhang, "Optical detection of ultrasound in photoacoustic imaging," IEEE Trans. Biomed. Engr. 64(1): 4-14 (2017)

[55] N. Huynh, E. Zhang, M. Betcke, S. Arridge, P. Beard, and B. Cox, "Single pixel optical camera for video rate ultrasonic imaging," Optica 3(1): 26-29 (2016)

[56] C. Sheaff, and S. Ashkenazi, "Characterization of an improved polyimide-etalon alloptical transducer for high-resolution ultrasound imaging," 61(7): 1223-1232 (2014)

[57] P. Hajireza, K. Krause, M. Brett, and R. Zemp, "Glancing angle deposited nanostructured film Fabry-Perot etalons for optical detection of ultrasound" Opt. Expr. 21(5): 6391-6400 (2013) 